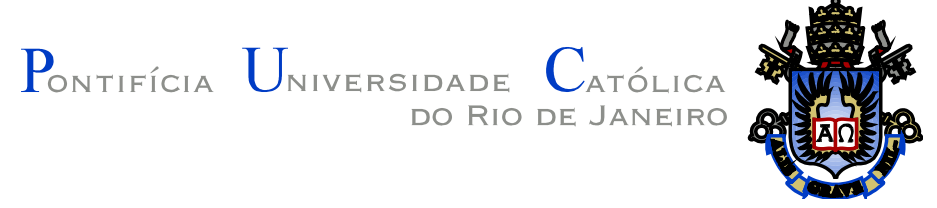

Victor Lopes Takahashi

Wireless ultrasonic energy and data transmission through fluid and metallic layers

Dissertação de Mestrado

Dissertation presented to the programa de Pósgraduação em engenharia mecânica of PUC-Rio in partial fulfillment of the requirements for the degree of Mestre em Engenharia Mecânica.

Advisor: Prof. Arthur Martins Barbosa Braga

Co-Advisor: Prof. Alan Conci Kubrusly

Rio de Janeiro

October 2017 
Victor Lopes Takahashi

\section{Wire less ultrasonic energy and data transmission through fluid and metallic layers}

Dissertation presented to the programa de Pósgraduação em engenharia mecânica of PUC-Rio in partial fulfillment of the requirements for the degree of Mestre em Engenharia Mecânica. Approved by the undersigned Examination Committee:

$$
\begin{array}{r}
\text { Prof. Arthur Martins Barbosa Braga } \\
\text { Advisor } \\
\text { Departamento de Engenharia Mecânica - PUC-Rio } \\
\text { Prof. Alan Conci Kubrusly } \\
\text { Co-Advisor } \\
\text { Centro de Estudos em Telecomunicações - PUC-Rio }
\end{array}
$$

Prof. Julio Cezar Adamowski

Departamento de Engenharia Mecatrônica e de Sist. Mecânicos - USP

Dr. Miguel de Andrade Freitas

Centro de Pesquisa em Tecnologia de Inspeção - PUC-Rio

Prof. Márcio da Silveira Carvalho

Vice Dean of Graduate Studies

Centro Técnico Científico - PUC-Rio

Rio de Janeiro, October 11th, 2017 
All rights reserved.

\section{Victor Lopes Takahashi}

The author graduated in Electrical Engineering from PUC-Rio in 2014. Currently works in the Laboratório de Sensores à Fibra Óptica as Research Engineer.

Bibliographic data

Takahashi, Victor Lopes

Wireless ultrasonic energy and data transmission through fluid and metallic layers / Victor Lopes Takahashi ; advisor: Arthur Martins Barbosa Braga ; co-advisor: Alan Conci Kubrusly. - 2017. 117 f. : il. color. ; $30 \mathrm{~cm}$

Dissertação (mestrado)-Pontifícia Universidade Católica do Rio de Janeiro, Departamento de Engenharia Mecânica, 2017. Inclui bibliografia

1. Engenharia Mecânica - Teses. 2. Ondas ultrassônicas. 3. Comunicação por ultrassom. 4. Modelo analítico. 5. Modelo elétrico. I. Braga, Arthur Martins Barbosa. II. Kubrusly, Alan Conci. III. Pontifícia Universidade Católica do Rio de Janeiro. Departamento de Engenharia Mecânica. N. Título. 
Aos meus pais, Ana Beatriz e George, pela entrega e dedicação. 


\section{Acknowledgment}

I would like to dedicate this work to my parents for the commitment and dedication throughout life.

To my sister, Ivana, for the eternal partnership and friendship.

To my advisor, Arthur Braga, for the professional and academic support, also for the past ten years of conviviality at the Optic Fiber Sensor Laboratory.

To my co-advisor, Alan Kubrusly, for the patience, dexterity and extreme wisdom conducting his academic insights.

To my friend, Sully Quintero, for the full dedication as a professional and for the great fellowship in life practice.

To my partner in life, Ana Luiza, for her positive energy and to show me the way to my own selfknowledge.

To my friend, Sávio Weslley, for the many hours of intense academic debating, for the support to my academic activities and also for his friendliness.

To PUC-Rio for the aid granted, which I could not accomplish this work without. 


\section{Abstract}

Takahashi, Victor Lopes; Braga, Arthur Martins Barbosa (Adviser); Kubrusly, Alan Conci (Co-adviser). Wireless ultrasonic energy and data transmission through fluid and metallic layers. Rio de Janeiro, 2017. 117p. Dissertação de Mestrado - Departamento de Engenharia Mecânica, Pontificia Universidade Católica do Rio de Janeiro.

Nonintrusive power transfer and data communication between devices through metallic walls is an increasing need in several sensing systems. Traditional means of communication mainly use electric conductors or electromagnetic waves. The first needs some mechanism for penetration whereas the latter, although nonintrusive, can be extremely limited due to the Faraday shielding effect. An alternative is found in the use of acoustic waves to transfer data and energy through metallic walls. Although great effort has been recently directed towards this type of communication, there still is a shortage of data dealing with the acoustic channel in the presence of multiple layers as well as of experimental results with curved metallic walls. Possible applications in these contexts may be found when monitoring pressure vessels filled with a fluid or pipes conveying liquids. The present dissertation evaluates, analytically, numerically and experimentally, the transmission of energy and data communication through a multi-layered, liquidmetal acoustic channel, composed of two curved metallic walls with a layer of liquid between them. For this, initially, two models based on propagation of ultrasonic waves are analyzed and compared, one analytical and the other numerical, both relying on electric-acoustic analogies. Both are extended to include more than one layer of material. The energy efficiency assessment and data transfer capability are addressed through the models and also experimentally validated using an acoustic channel comprising a flat aluminum plate and two axially aligned piezoelectric transducers coupled to it. In addition, an electric circuit is developed for the transmission of energy from outside to inside and the communication of digital data from the inside to the outside by ASK modulation and demodulation. The circuit is simulated using electrical circuit simulation soffware, designed and assembled with printed circuit boards. Thereaffer, a second experiment where the acoustic channel is composed by a curved metallic section with an intermediate 
fluid layer is implemented. In this, the power and data transfer are studied using the developed electric circuit, which is connected to a pair of piezoelectric transducers coupled to the acoustic channel. Results for the flat aluminum plate reveal good agreement between both models and the experiment, both by frequency and time domain analysis. The analytical model best reproduced the physical phenomenon of interest due to its stricter treatment of loss mechanisms. The second experiment proved the feasibility of multi-layered liquid-metal communication on curved walls and showed that the system is able to transmit data from temperature and pressure sensors at a rate of $9600 \mathrm{bps}$. The sensor and all its peripheral circuitry were fully powered by the energy flowing through the acoustic channel in total of approximately $140 \mathrm{~mW}$.

\section{Keywords} model.

Ultrasonic waves; Ultrasonic communication; Analytic model; Electric 


\section{Resumo}

Takahashi, Victor Lopes; Braga, Arthur Martins Barbosa; Kubrusly, Alan Conci. Comunicação sem fio e transferência de energia através de paredes metálicas e camada de líquido utilizando ondas ultrassônicas. Rio de Janeiro, 2017. 117p. Dissertação de Mestrado - Departamento de Engenharia Mecânica, Pontifícia Universidade Católica do Rio de Janeiro.

Existe uma crescente necessidade em transferir energia e comunicar dados entre dispositivos através de paredes metálicas de forma não intrusiva. Os meios de comunicação tradicionais para este fim, em sua maioria, baseiam-se essencialmente no uso de condutores elétricos ou ondas eletromagnéticas. O primeiro necessita de algum mecanismo de penetração e o segundo, apesar de não intrusivo, torna-se limitado devido ao efeito de blindagem de Faraday. Uma alternativa é encontrada no uso de ondas acústicas para transferir os dados e energia através de paredes metálicas. Recentemente, grande esforço tem sido empregado nesse tipo de comunicação, todavia há ainda carência de trabalhos que tratem do canal acústico na presença de multicamadas além de resultados experimentais com paredes metálicas curvas. Possíveis aplicações nestes contextos são encontrados no monitoramento de vasos de pressão com fluido no seu interior ou até mesmo de tubulações metálicas transportando líquidos. A presente dissertação avalia, de forma analítica, numérica e experimental, a transmissão de energia e a comunicação através de um canal acústico composto por camadas metal-líquido-metal com paredes curvilíneas. Para tal, inicialmente, são analisados e comparados dois modelos, existentes na literatura, fundamentados na propagação de ondas ultrassônicas, um analítico e outro numérico, ambos baseados em analogias acustoelétricas. Os dois modelos são estendidos permitindo a inclusão de múltiplas camadas de diferentes materiais. A avaliação da eficiência de energia e a capacidade de transferência de dados é fita com base nos modelos e validada experimentalmente utilizando uma placa reta de alumínio e um par de transdutores piezoelétricos axialmente alinhados e acoplados ao mesmo. Um circuito elétrico é desenvolvido para a transmissão de energia entre as duas faces da placa e para a comunicação de dados digitais por meio de modulação do tipo ASK. O circuito é então simulado utilizando-se um software de simulação de circuitos elétricos, 
projetado e montado com placas de circuito impresso. Em seguida, é realizado um segundo experimento utilizando uma seção curva metálica com uma camada intermediária de água como canal acústico . Nesse, são estudadas as transferências de energia e dados utilizando o circuito elétrico desenvolvido o qual é conectado a um par de transdutores piezoelétricos acoplados ao canal acústico. Resultados do experimento na placa reta de alumínio revelam boa consonância entre os modelos e o experimento, tanto por uma análise em frequência quanto no domínio do tempo. Tendo sido o modelo analítico o que melhor representa o fenômeno físico em questão devido ao maior rigor no tratamento do mecanismo de perdas. Para o segundo experimento, resultados comprovam a possibilidade de comunicação através de múltiplas camadas metálicas e líquidas em paredes curvas, mostrando que o sistema é capaz de transmitir dados de um sensor de temperatura e pressão a uma taxa de 9600 bps. Tanto o sensor quanto os seus circuitos periféricos são integralmente alimentados pela energia que atravessa o canal acústico, num total de aproximadamente $140 \mathrm{~mW}$.

\section{Palavras-chave}

Ondas ultrassônicas; Comunicação por ultrassom; Modelo analítico; Modelo elétrico 


\section{Table of Contents}

1 Introduction 15

1.1 Objective 18

1.2 Dissertation contribution 18

1.3 Dissertation layout $\quad 19$

2 Bibliography review 21

3 Analytical and numerical design 35

3.1 Pspice modeling 35

3.1.1 Piezoelectric transducer model 35

3.1.2 Intermediate layer model $\quad 41$

3.1.3 Pspice loss considerations 44

3.1.4 Full system model $\quad 47$

3.1.5 Analysis of power transfer $\quad 49$

3.1.6 Analysis of data communication $\quad 50$

3.2 Analytical modeling $\quad 51$

3.2.1 Two-port ABCD matrix $\quad 52$

3.2.2 Piezoelectric transducer model 53

3.2.3 Intermediate layer $\quad 56$

3.2.4 Analytical loss considerations 58

3.2.5 Analysis of power transfer 61

3.2.6 Analysis of data communication 62

3.3 Models verification and comparison 64

4 PDAC implementation $\quad 70$

4.1 ASK $\quad 70$

4.2 Outside block circuit $\quad 71$

4.3 Inside block circuit $\quad 74$

4.4 Full system simulations $\quad 76$

4.5Full system simulation results $\quad 76$

4.5.10utside block simulation $\quad 78$

$\begin{array}{ll}4.5 .2 \text { Inside block simulation } & 79\end{array}$

4.6 Experimental setup $\quad 81$

4.6.1 Flat plate $\quad 82$

4.6.1.1 Flat plate power $\quad 84$

4.6.1.2 Flat plate communication analysis 86

4.6.2 Curved surface - Tube 90

4.6.2.1 Curved surface power transfer analysis 93

4.6.2.2 Curved surface data communication analysis 94

4.6.3 Full system experimental analysis - Tube 97

5 Conclusion 104

6 Bibliographic reference 107 


\section{Figure list}

Figure 1.1 "sandwiched" PZT transducers between a metal layer, redrawn from [63]

Figure 1.2 Diagram of power and data communication through acoustic channel. Power is provided from the outside block to the inside block whereas sensor information is transmitted from the inside block to the outside block.

Figure 1.3 a) Flat plate with the PZT transducers (red rectangles) coaligned b) Curved surface in the presence of water

Figure 2.1 Diagram developed by Imoru et al. [19] to evaluate the power transfer through a metal pipe. Reproduced from [19].

Figure 2.2 A pipe made of stainless steel with an internal coil and an external coil that entirely enclose the pipe, redrawn from [20]

Figure 2.3 Basic configuration of a capacitive power transfer, redrawn from [22]

Figure 2.4 Configuration of $2 \mathrm{PZT}$ trans ducers co-aligned an attached to the metal wall, redrawn from [67]

Figure 2.5 (a) Single-hop (b) Double-hop and (c) reflected-power configuration, redrawn from [43]

Figure 2.6 Power and data communication of a sensor inside metallic envelope, redrawn from [53]

Figure 3.1 Overall diagram of PDAC generic system

Figure 3.2 Diagram of the piezoelectric transducer

Figure 3.3 (a) Transmission line representing the mechanical part controlled by the current (i) and (b) the electrical part of the transducer controlled by the particle velocity

Figure 3.4 Transducer subcircuit schematic in ORCAD software

Figure 3.5 Spreading attenuation of acoustic wave

Figure 3.6 Full system circuit designed in ORCAD

Figure 3.7 Acoustic channel with S11 and S21 blocks represented in blue and red, respectively

Figure 3.8 Two-port configuration of S-parameters 
Figure 3.10 Piezoelectric mechanical and electrical ports, redrawn from [63]

Figure 3.11 Equivalent impedance diagram 63

Figure 3.12 Power transfer analysis of both models varying the metal thickness 65

Figure 3.13 Power transfer analysis varying the adhesive thickness $\quad 66$

Figure 3.14 Input voltage V11 Pspice and analytical 67

Figure 3.15 V11 difference Pspice and analytical 67

Figure 3.16 Output PZT voltage at $2.256 \mathrm{MHz} 68$

Figure 3.17 Output PZT voltage at $2.235 \mathrm{MHz} 68$

Figure 4.1 ASK modulation diagram $\quad 71$

Figure 4.2 Envelope detector schematic 72

Figure 4.3 Envelope Signal and Modulated signal example 72

$\begin{array}{ll}\text { Figure 4.4 Voltage comparator diagram } & 73\end{array}$

Figure 4.5 input and output signals of the voltage comparator $\quad 74$

Figure 4.6 Voltage doubler topology 75

$\begin{array}{ll}\text { Figure 4.7 Mosfet switching RF carrier } & 76\end{array}$

Figure 4.8 PDAC full system with electronic peripherals circuits $\quad 77$

Figure 4.9 Modulated signal of full system 78

Figure 4.10 Sensor signal of full system 78

Figure 4.11 Comparision between sensor information and comparator signal $\quad 79$

Figure 4.12 Inside PZT signal $\quad 80$

Figure $4.135 \mathrm{~V}$ voltage regulator output 81

Figure 4.14 Impedance curve for Pspice, Analytical and experimental $\quad 85$

Figure 4.15 Schematic (a) and experimental (b) characterization of the flat plate using VNA 85

Figure 4.16 Flat plate power analysis $\quad 86$ 
Figure 4.17 Setup for data communication analysis in frequency domain

Figure 4.18 Voltage divider theory

Figure 4.19 (a) Input voltage (V11) varying the termination load and zoom (b) between 2.1 and $2.5 \mathrm{MHz}$

Figure 4.20 Difference in amplitude voltage 88

Figure 4.21 Setup to measure the input voltage (V11) 89

Figure 4.22 Input voltage signal flat plate experimental test 90

Figure 4.23 Three-dimensional sketch of curved surface experiment $\quad 92$

Figure 4.24 3D CAD and Structure made on 3D printer to isolate the back face of transducer from water 93

Figure 4.25 Power transfer analysis for curved surface experiment 93

Figure 4.26 Input voltage (V11) - curved surface 94

Figure 4.27 Input voltage between $0.95 \mathrm{MHz}$ and $1.05 \mathrm{MHz}-$ curved surface

Figure 4.28 Input voltage difference between 50 and $0.22 \mathrm{ohms,} \mathrm{full}$ frequency range

Figure 4.29 Input voltage zoom for frequencies between $1 \mathrm{MHz}$ and $1.05 \mathrm{MHz}$

Figure 4.30 Input voltage transient analysis - Curved surface

Figure 4.31 Schematic diagram of the curved surface experiment test 98

Figure 4.32 Full system experimental bench

Figure 4.33 Sensor information signal in green and the input voltage signal on the outside block in purple

Figure 4.34 Block diagram of protocols used in the inside block

101

Figure 4.35 Sensor information signal in green and purple the signal after the voltage comparator in purple

Figure 4.36 Comparison between raw and demodulated temperature sensor data

Figure 4.37 Bubbles generated inside the water due to cavitation-like phenomenon 


\section{Table list}

Table 3.1 characteristic impedances of common materials

Table 3.2 Analogies between mechanical and electrical of PZT transducer

Table 3.3 Analogies between mechanical and electrical of intermediate layers

Table 3.4 Materials properties 48

Table 3.5 2-port S-parameters 50

Table 4.1 Material properties 84

Table 4.2 Power Transfer of Pspice, analytical and experimental 86

Table 4.3 Highest difference in amplitude 89

Table 4.4 Material properties - Tube 91

Table 4.5 Power transfer peak of curved surface experimental setup 94

Table 4.6 Voltage difference of the input voltage 95

Table 4.7 Power consumption of the main elements

in the inside block 


\section{Introduction}

Wireless communications play a key role in integrating a sensor network. Some communication protocols have become quite popular in recent decades such as Wi-Fi, Bluetooth, 3G, 4G, among others [1]. All ofthese protocols, however, use electromagnetic waves in order to communicate and in some applications, such as in the presence of metal walls, for instance, the performance of the communication suffers a great decline mainly because of the Faraday shield phenomenon [2]. As an alternative, acoustic waves use the material medium to propagate mechanical waves by oscillating stress/strain field inside the material [3]. Typical applications where an enclosure metal is present is at ship containers [4], submarines [5] in hazardous environment $[6,18]$, or even on a wellbore casing, exposed to high pressure and temperature in which sensors are interrogate through the wellbore wall [89]. A common scenario consists of sensors located outsidethe hull of a submarine or inside the container; these sensors need to send data through the metal wall, which cannot be penetrated [5]. The sensor maintenance and the associated peripherals circuits are offen very laborious or unfeasible, relying on remote wireless powering system can be of interest to avoid replacing batteries or power supplies. Recently, great attention has been drawn by research groups from over the globe to system which combine data communication and power transmission by means of acoustic waves [4-10].

The most typical appl ication reli es on the configuration of two sandwich-struct ured PZT transducers between a metal layer attached to the wall with epoxy or other acoustic couplant as shown in Fig. 1.1. Using this configuration, results from literature shows power efficiency varying from $30 \%$ to $88 \%[5-7,12-15]$ wi the thickness of the metal barrier wall going from $1.6 \mathrm{~mm}$ to $63.5 \mathrm{~mm}$. When speaking on the data transfer capability, several data transmission protocols were implemented, for instance FSK[4], ASK[6, 7, 14], OFDM[5,16], QPSK[15]. Data rate varies dramatically fom lower $1 \mathrm{kbps}$ up to $17.37 \mathrm{Mbps}$, using OFDM.

Despite this, few works implemented a complete system integrating sensors and hardware design [14]; it is also not common to find works reporting analysis of power and data transfer with multiple layers, especially including a fluid layer. [17] 


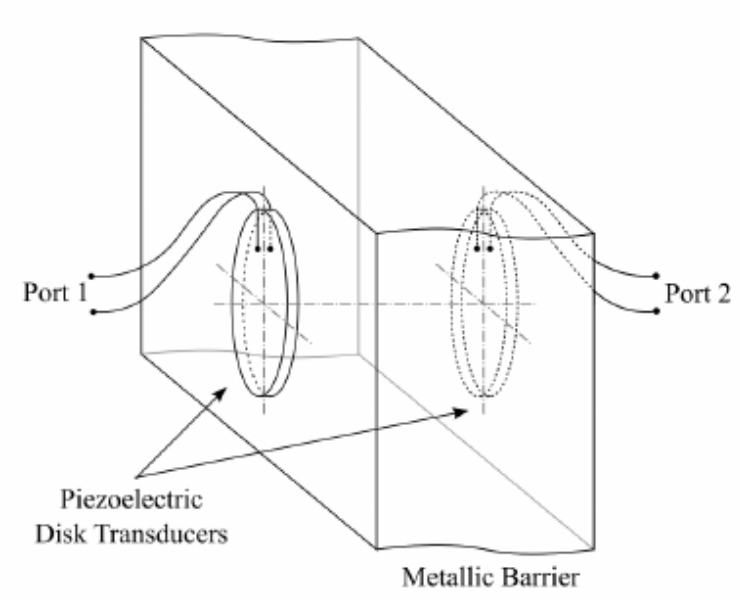

(a) Isometric View

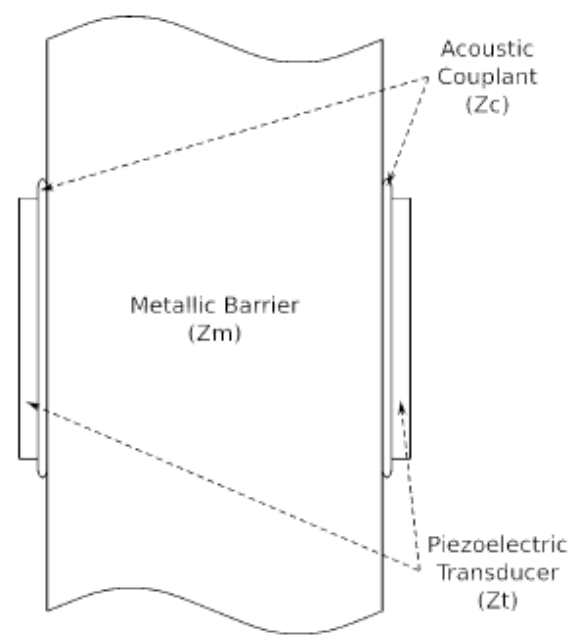

(b) Cross-Section (Components are Not to Scale)

Figure 1.1 sandwich-structured PZT transducers between a metal layer, redrawn from [63]

The overall system to power and data communication through acoustic channel, (PDAC) typically contains three major blocks: the "outside block", "inside block" and the "acoustic channel". These definiti ons were primary presented by Prada in [7] and maintained in this dissertation. The "outside block" encompasses the peripherals circuitry and accessories to create the carrier wave that drives the system and to demodulate the data information from the sensors, connected to the inside block. The "inside block" works passively and powers the sensors using exclusively the energy received from the "outside block". The communicati on of the sensors is performed modulating the carrier wave generated in the "outside block". Finally, the "acoustic channel block" consists on the material medium (which can be composed by many layers, such as metals, water, and adhesive) and two PZT transducer. Fig. 1.2 illustrates the block diagram of the system described. This configuration has the advantage of been simple to implement and capable of communicating with baud rates which can reach moderated speeds; the power received in the "inside block" is sufficient to power sensors and peripheral electronics with low consumption [14]. Additionally this design allows the inside block to be inaccessible and to work indefinitely since its power is supplied fom outside and ideally it needs no external intervention. 


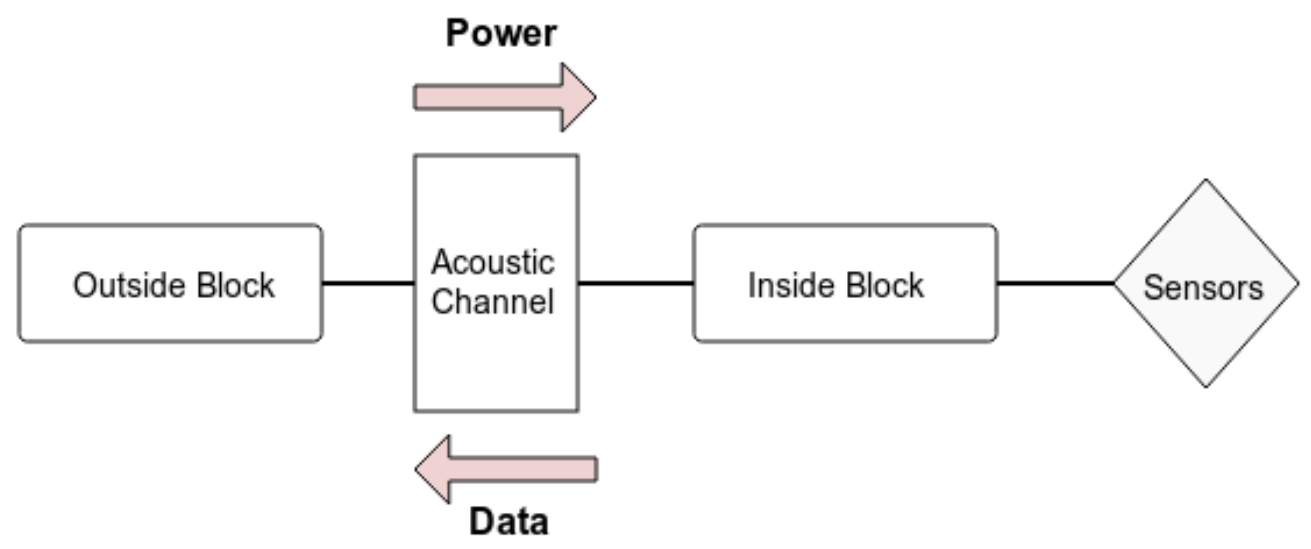

Figure 1.2 Diagram of power and data communication through acoustic channel. Power is provided from the outside block to the inside block whereas sensor information is transmitted from the inside block to the outside block.

In this dissertation a system which uses the acoustic waves to power and communicate sensors is investigated by means of numerical model and experiments. Two model s are described and compared, one analytical and other numerical based on electric circuit simulation soffware. These two models aid in evaluating the power transfer efficiency between the PZT transducers and data communication capability of the system. Furthermore, experimental tests were performed to compare and validate the results of the models and evaluate the designed hardware.

Two different setups were eval uated here. The first consists of a metal flat plate with two PZT transducers attached by an epoxy gl ue and geometrically coaligned, Fig 1.3 (a); the other is a curved surface with the two PZT transducers al so co-aligned and in the presence of water, Fig. 1.3 (b). The former, above all, was implemented to validate the concept of the PDAC and the latter, concedes the evaluation of a fill system in such applications that include a fuid layer and a curved surfice. The curved configuration is of interest because it could be any vessel or concentric pi pes immersed in water, in which sensors located inside the inner tube or outside the outer one are not accessible. Thus, this last configuration can be of potential interest in practical monitoring systems. 

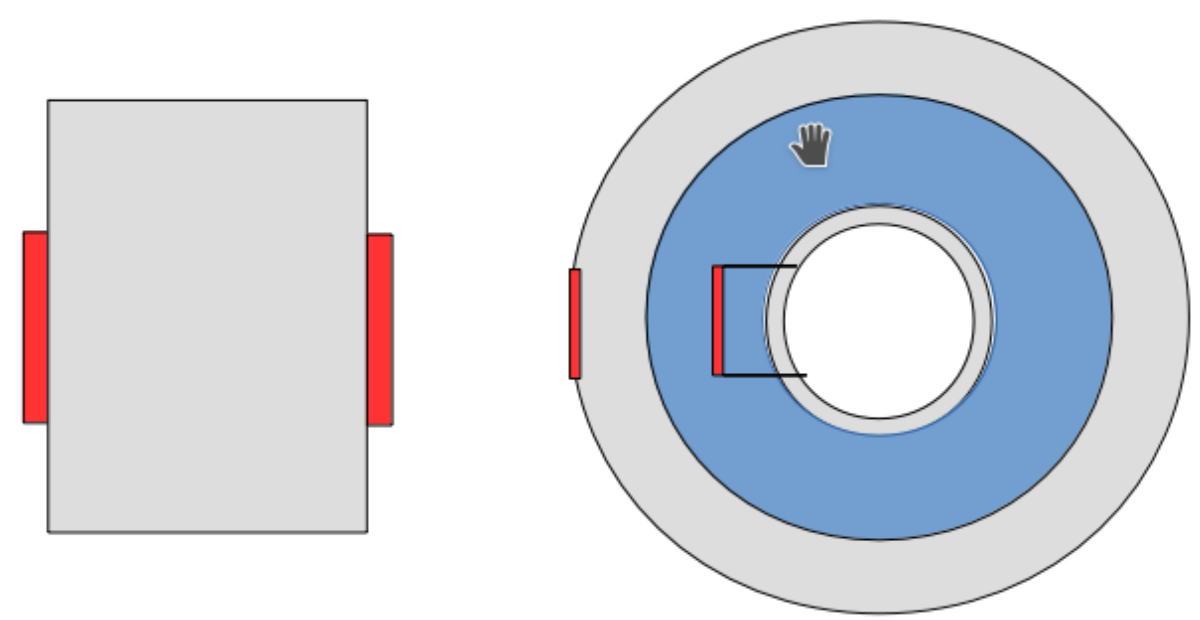

Figure 1.3 a) Flat plate with the PZT transducers (red rectangles) co-aligned b) Curved surface in the presence of water

The two setups of figure 1.3 were evaluated by the analytical and numerical models and the results compared with each other and with an experimental setup. Finally, the full system connecting one pressure and temperature sensor, communicating and powering through water layer under a curved steel surface was successfully tested in laboratory conditions.

\section{1 \\ Objectives}

The main goal of this dissertation is to design and create a system that powers and communicates a sensor located in the inside block by means of acoustic waves, in which the acoustic channel is composed by multiple fluid and solid curved layers. Specific objectives are to evaluate and extend two pre-existing models, including multilayer, and to assess a comparison between them and experiments.

\section{2}

\section{Dissertation contribution}

This dissertation provides the fol lowing contribution to the existing literature:

- Comparison of analytical and numerical models between them and between experiments for a single and multi-layer with fuid, by previous extending the models to incl ude multiple layers as the acoust ic channel . 
- Simulation, design and evaluation of the PDAC peripherals circuits integrated to a sensing system through curved surface in presence of water layer.

As a bottom line, this is the first work regarding power and data communi cation using acoustic waves for the research group, all owing the use of this research theme and mainly these results to build a framework for further works.

\section{3}

\section{Dissertation layout}

The dissert ation is organized as follows:

1. Introduction:

Introduces the work.

2. Bibl iography review:

Presents a bibli ography review and state of the art for methods to wi reless transmit power and data through metal layers. The review is focused in acoustic waves' solutions but also presented el ectromagnetic approaches for a complete understanding of the problem.

\section{Analytical and numerical design:}

This chapter outlines the overall system proposed for the PDAC. A numerical model based on Pspice and an analytical formulation is revi sited to describe the PZT transducer and intermediate layers and their associated losses. Simulation results in fequency and timedomain and a comparison of both models with a brieffy discussion are presented.

\section{PDAC implementation:}

This chapter presents the discussion about the modulation used and a detailed description about the outsi de and inside circuit blocks. A fill system with the outside, inside and acoustic channel blocks are simulated in Pspice and the results di scussed. The circuits of insideand outside block, which were designed and mounted in printed circuit boards, are presented. They are responsi ble to modul ate and demodulated the sensors data and power the inside block. Two experi ments are presented, the first with the flat plate and the other with the curved surface in 
presence of a fuid. The latter was al so used in a fill pressure and temperature sensing system.

5. Discussion and Future Work:

Conclusion with a final di scussion of the overall system and a brief out line for fiture work. 


\section{2 \\ Bibliography review}

Methods to wireless transmit power and data through a channel can be split in two major blocks; based on electromagnetic waves and acoustic waves. Although the main objective of this dissertation is related with acoustic waves an overview on methods to transmit power and data wirelessly including electromagnetic waves is essential to outline and compare the crucial points of operation. In this survey, however, not all the publications specifically concern transmission of power and data simultaneously.

\section{1 \\ Wire less power and data transmission based on electromagnetic waves}

Regarding electromagnetic waves some publications stand out. In the first, Imoru at al. [19] use the principle of inductive coupling to transfer power through metal. This principle is similar to the coupling oftransformers. In a transformer, the energy is transferred from one coil to another. The primary coil produces an alternative magnetic field that induces a current on the secondary coil by the principle of electromagnetic induction. The configuration developed by [19] is represented in figure 2.1, in which two coils are winded around a pipe section, one inside and other outside. The outer coil transfer power to the inner one, by inducing a current in the latter which passes through a resistor. In this publication the author reports a maximum efficiency of $23 \%$ with a load of $5.55 \Omega$ operating at $100 \mathrm{~Hz}$ in $32.5 \mathrm{~mm}$ of distance between the radius of each coil. 


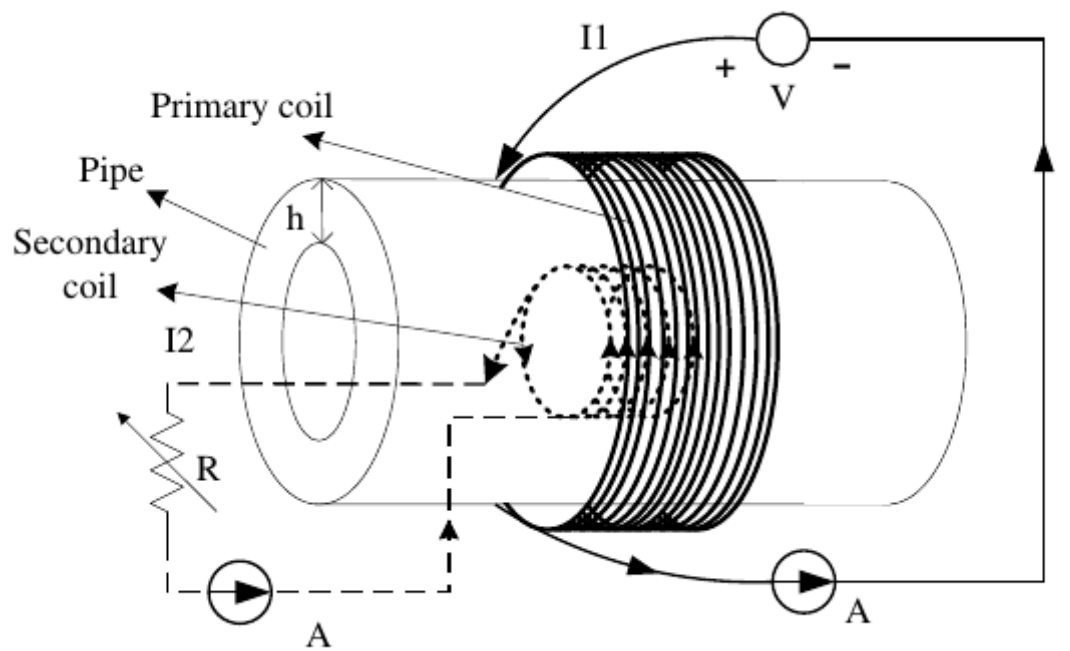

Figure 2.1 Diagram developed by Imoru et al. [19] to evaluate the power transfer through a metal pipe. Reproduced from [19].

This solution can even transmit data, however with extremely low data communication, at about $10 \mathrm{bps}$.

Zangl et al. [20-21], designed an experiment consisting of a steel pipe with an internal coil connected to a capacitive sensor and an external coil that can entirely enclose the pipe in order to maximize the power transfer efficiency, see Fig. 2.2. With $2 \mathrm{~mm}$ of pipe wall thickness the magnetic fux density decrease $15 \mathrm{~dB}$. At $50 \mathrm{~Hz}$ the system achieve $20 \mathrm{~dB}$ of signal to noise ratio when applying $540 \mathrm{~mA}$ on the external coil. The author also commented the feasibility to transmit data at more than 20 kbps.

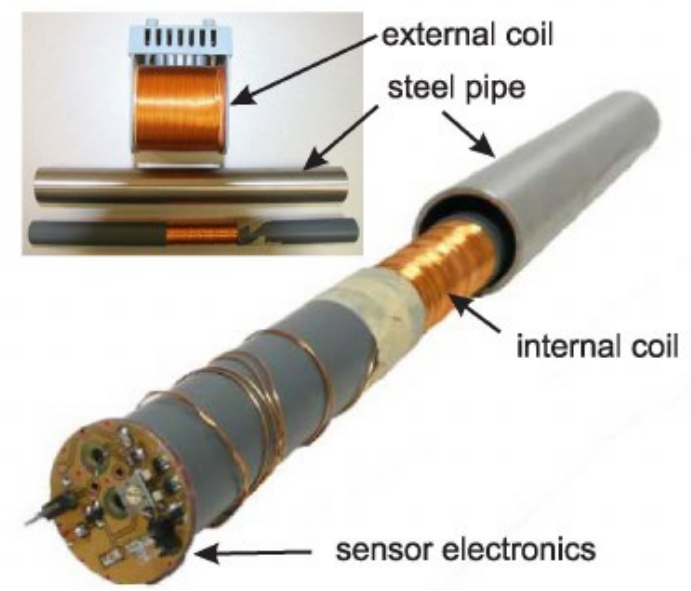

Figure 2.2 A pipe made of stainless steel with an internal coil and an external coil that entirely enclose the pipe, redrawn from [20] 
Other type of configuration to transfer power is the use of electric field instead of magnetic field, which is established by means of capacitive coupling. Unlike the inductive coupling which is based on magnetic field coupling to transfer power, the capacitive coupling uses the electric field coupling. The configuration of a typical capacitive coupling has two pairs of plates in which an AC voltage is applied. When two separated pair of plates are placed close to the primary pair, electric fields are formed. In between these two pairs of plates a metal wall can be applied. Liu and Chao [22] presented this configuration where the coupling distance of each pair was $1 \mathrm{~mm}$, see Fig. 2.3. The input power was set to $28.8 \mathrm{~W}$ while the output power had a decrease down to $12.3 \mathrm{~W}$, i.e. achieving $42.7 \%$ of efficiency.

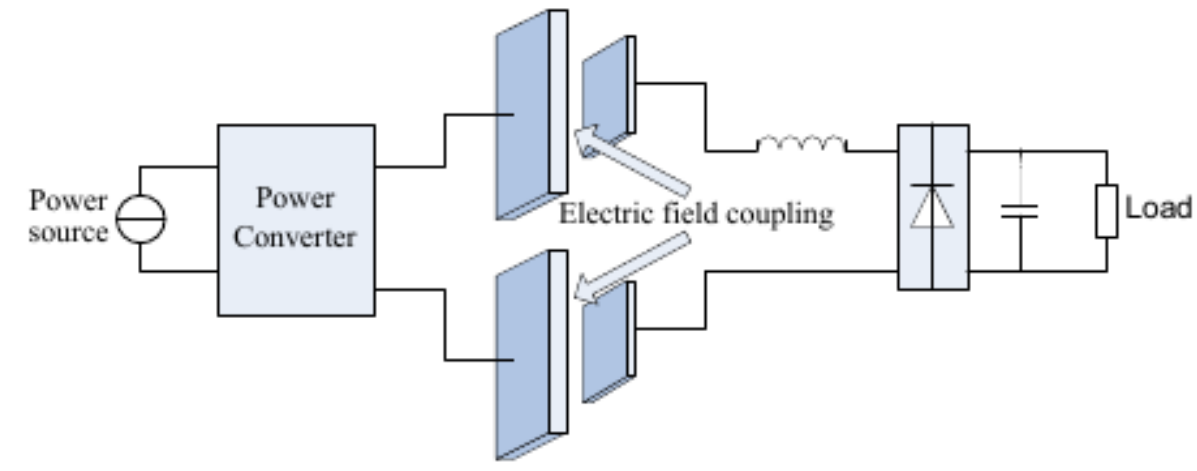

Figure 2.3 Basic configuration of a capacitive powertransfer, redrawn from [22]

In [23] Graham has developed experiments to evaluate the transfer of power and data through metal barriers simultaneously and concluded that using the inductive coupling to power and communicate the sensor electronics is limited to lower rate, few bps, due to the narrow the bandwidth. Experiment showed that up to $100 \mathrm{bps}$ can be transmitted when a stainless steel plate of $20 \mathrm{~mm}$ is in the middle of the coils.

It can be summarized that it is possible to wirelessly transmit power and data with electromagnetic waves. However, the power efficiency and the data rate communication are strongly affected by the low RF penetration inside the material [24] and the operation frequency. The propagation using acoustic waves, otherwise, does not suffer such specific attenuation through metal barriers, as electromagnetic wave does, becoming then attractive for use in power and data transfer. 


\section{2 \\ Wire less power and data transmission based on acoustic waves}

Communications using acoustic waves have first started in 1945 at the Naval Underwater Sound Laboratory. This first application was developed to communicate with submerged submarines [25] using a carrier wave of $8.3 \mathrm{kHz}$. This solution was adopted because electromagnetic waves are extremely absorbed in conductive medium such as sea water. The absorption rate in this condition is 45 $\sqrt{f} \mathrm{~dB}$ per kilometer, where $\mathrm{f}$ is in Hertz. That publications also shows that the attenuation is much lower for the acoustic waves, approximately three orders of magnitude. Other application widely used which helped to boost the growth of acoustic waves, mainly the ultrasonic waves, was the biomedical industry, for instance, the ultrasound-based diagnostic imaging technique (ultrasonography) [26] and wireless power and data communication of devices located inside the human body [27]. Comparing with the methods to communicate through a metal layer, the biomedical application and the underwater acoustic communication already have a long period of research, being in a mature state of development [124-128].

The first design which used the ul trasonic waves to power and communicate with sensors through metal was presented in a Connor et al. Patent in 1997 [90]. This is comprised by two co-ali gned PZTs attached on a solid metal wall, the outside PZT transducer is excited with a conti nuous wave (CW) electrical signal, the transducer convert the electrical signal into ultrasonic vibrations that propagates through the metal. The second PZT transducer located inside, receives the ul trasonic wave and re-converts it to an electrical signal. The energy received in the insi de transducer powers a sensor and its associated electronics. The communi cation of the sensor data between the inside and the outside is performed using the principle of impedance modulation. A transistor is connected in parallel with the electrical terminals of the inside transducer, acting as an electronic switch. Opening and closing the transistor changes the acoustic impedance of the PZT transducer, reffecting more orless acoustic energy. The variation in theimpedance impacts the amplitude of the CW carrier which in turn can be interpreted as an amplitude modulation. By this means, the sensors can transmit the information which is demodulated by an outside circuit. Nevertheless, the author does not provide 
characterization about the power efficiency and data rate capability neither the hardware used to modulate and demodul ate the sensor data.

Many research groups $[12,17-51]$ utilize the configuration of a pair of PZTs coaxially aligned and attached directly to opposite sides of the metal layer. Fig. 2.4 shows a diagram of this configuration. Moreover, these works have different approaches. Some of them focus on transmit power alone [28-40, 45], while others focus on transmit data alone [41-44,46-52], and in some case they focus on simultaneous transmit power and data [53-71].

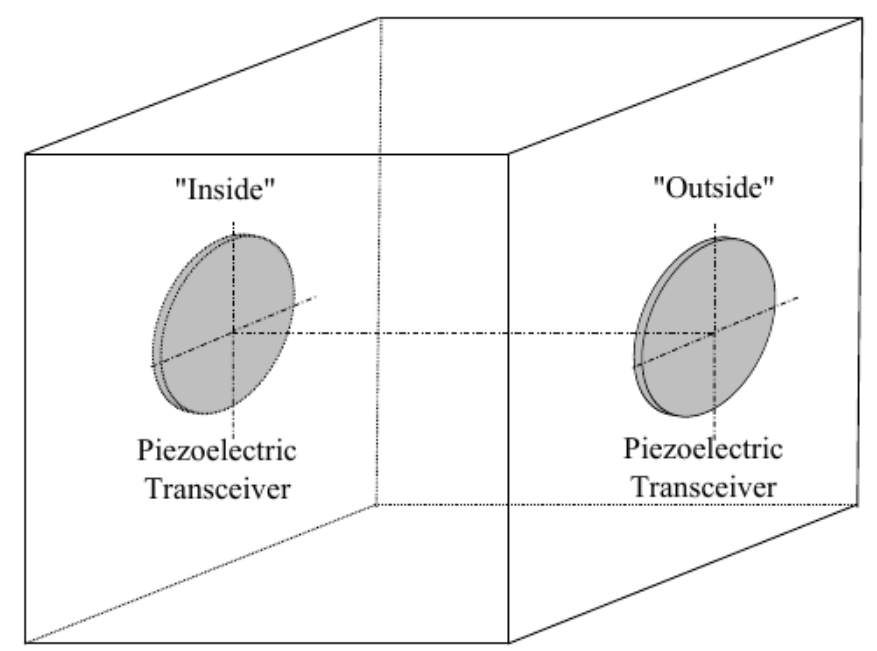

Figure 2.4 Configuration of 2 PZT transducers co-aligned an attached to the metal wall, redrawn from [67]

One of the first publication to analyze the use of PZTs to transmit energy through a metal layer was $\mathrm{Hu}$ et al. [28]. In this, they examined the feasibility of transmitting acoustic energy using the configuration of Fig. 2.4 without the need of coupling layer. The authors have presented a mathematical formulation for a thickness-stretch vibration when a harmonic electric voltage source drives the transmitting PZT. This study revealed that the voltage efficiency of the system is magnified at the first several resonant frequencies and the greatest magnification does not occur at the fundamental resonant frequency. Numerical simulations showed the behavior of the system varying the operating frequency and the input admittance when varying a load impedance connected to the receive PZT.

Next, several papers with physical and mathematical modeling of power transmission through metal layer using different transducers shapes and vibration modes were fllowed. In [29-30], a cylindrical metallic layer with a pair of shell 
shape piezoelectric transducers concentrically aligned on its inside and the outside surfaces is modeled by means of elastic and piezoelectric theory addressed in cylindrical coordinates. Thepaper al so shown that energy transfer is sensitive to the geometric and physical parameters of the structure. Hu et al. [31-32] showed that the power efficiency is dependent on the operating frequency, the mechanical properties of the PZTs and the electrical load viewed by the transducer. Therefore, a careful circuit design has to be developed to acquire good energy harvesting on the isolated side. These papers have addressed the problem by formulating mathematical models and verified them only by numerical results; no experimental tests were realized in them.

Sherrit et al. [33] proposed a network model of the acoustic-electric channel based on the equivalent impedance of the system elements (Piezoelectric transducer and metal layer) to study the power transfer efficiency based on Hu et al. [28] configuration. This model has the advantage of being easily expanded to account additional acoustic elements such as insulation layer and coupling layer.

In [34] different methods to attach the transducers on the metal were studied. The coupl ing methods include bolted with a backing structure, clamped with grease coupl ant and by attaching using a conductive epoxy. With 53\% of measured power efficiency the method using clamp and grease obtained the highest efficiency fol lowed by the conductive epoxy that gave $40 \%$ of efficiency.

Experimental results of $[35,36]$ showed that high power can be delivery through metal plates. Two experiments with distinct thickness of a metal barrier were evaluated, the first provides $100 \mathrm{~W}$ through $3.4 \mathrm{~mm}$ thick titanium plate and the second provides $1083 \mathrm{~W}$ through a $5 \mathrm{~mm}$ thick metal barrier. The latter is the highest reported power transfer achieved on the literature. This was possible by using a stack of four PZT s with a total thick of $13.6 \mathrm{~mm}$ in which the system present its resonant fequency at $24.5 \mathrm{kHz}$ obtaining an efficiency of $84 \%$.

Kiziroglou et al. [45] have transmitted power along a pipe with $1 \mathrm{~m}$ ofdistance between PZT s transducers, using acoustic transversal waves. The used PZT disk transducer has a nominal radial resonance of $50 \mathrm{kHz}$ and the system was able to deliver approximately $18 \mathrm{~mW}$ of power when $20 \mathrm{~W}$ is applied on the transmitter. An analysis of the power transfer in the presence of water inside the pipe shows a decrease of the power as the ffuid increases the attenuation of vibration throughout the spectrum, when using this configuration, mainly because part of the acoustic 
energy leaks to the water. Nevertheless, the system delivered $4 \mathrm{~mW}$ when $20 \mathrm{~W}$ was applied. Previously studies have shown that the power transfer is affected by the electrical reffection between the signal generator and the PZT transducer. In [60], a simultaneous conjugate impedance matching was analyzed as a method to improve the power transfer. The experimental results have shown a 55\% of efficiency with $81 \mathrm{~W}$ of power being transmitted through the channel.

Up to here the above references have focused on wireless power delivery through metal layers. Some research groups however developed studies mainly on data communication through metal for enclosed sensors. The first report to do such analysis is dated 2000 by Hobart et al [41]; they have developed a device for transmitting data through a ship's hull. Payton, on the other hand, creates a patent titled "System for acoustically passing electrical signals through a hull" [42] describing the use of two pairs of piezoelectric transducer in which one of them is in charge of forward transmission and the other of reverse transmission. The patent does not specify if an experimental test was realized.

At about the same time in 2006, a research group at Rensselaer Polytechnic Institute (RPI) initiated the study of wireless ultrasonic data transmission through metal layers. The first work is themaster's thesis of Murphy [43] which investigated methods for sensor monitoring insidea sealed pressure vessel. Three configurations were created; a "single-hop", Fig. 2.5 (a), which uses an unidirectional data transmission, a "double-hop", Fig. 2.5 (b), which is a full-duplex system with separated forward and reverse transmission, and a bidirectional data transmission named "reffected-power", Fig. 2.5 (c), which uses the variation of the transducer impedance to communicate. Data rate of $500 \mathrm{bps}, 5 \mathrm{kbps}$ and $300 \mathrm{bps}$ was respectively achieved for the three configurations on a $148 \mathrm{~mm}$ thick steel layer. Thereafter, Saulnier, et al [44] have developed a configuration that merges the "double-hop" and "reffected-power" configurations. This was able to communicate with a sensor at a rate of $500 \mathrm{bps}$ through a $152.4 \mathrm{~mm}$ thick steel. 


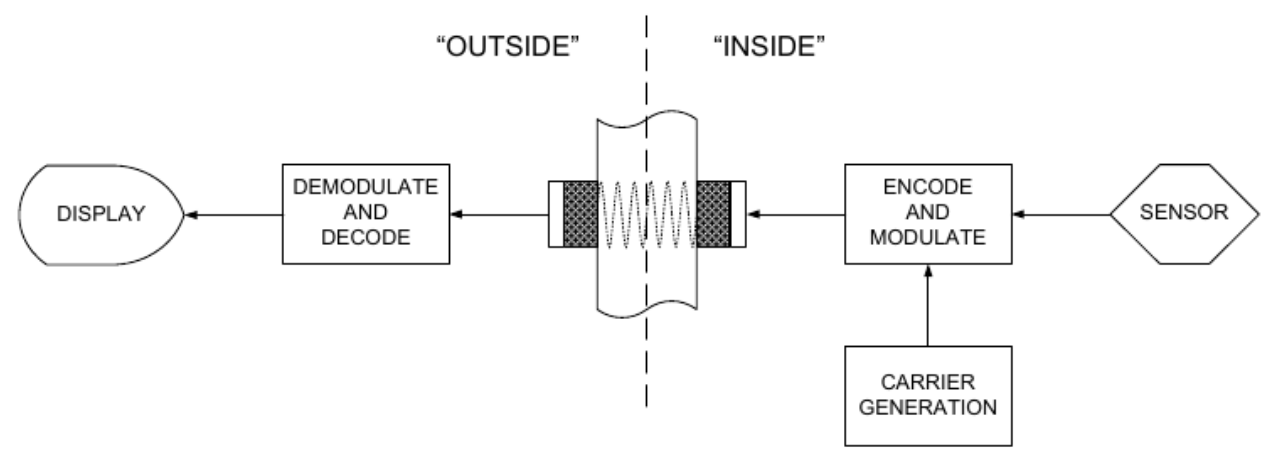

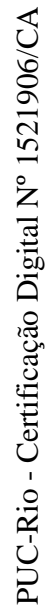
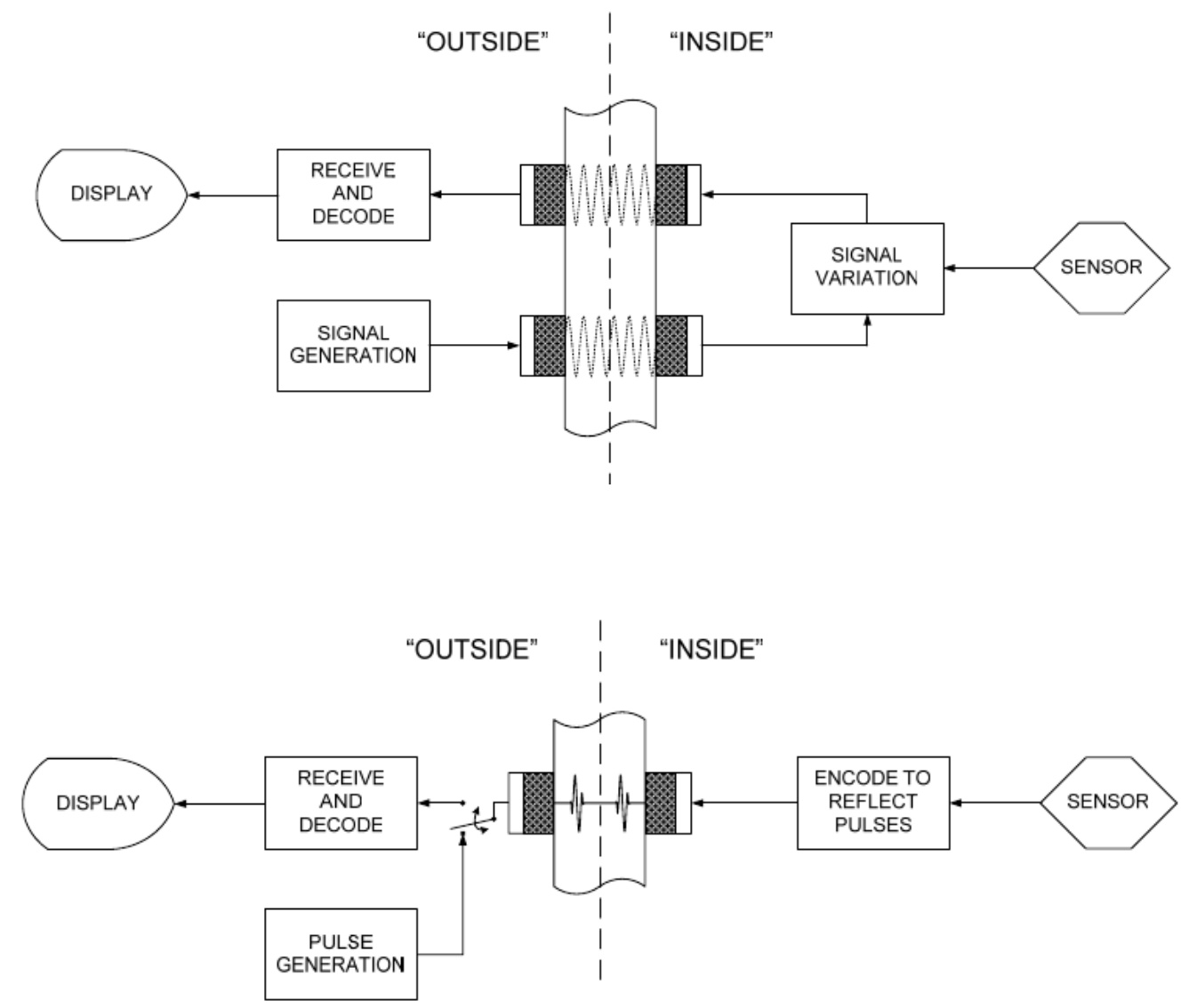

Figure 2.5 (a) Single-hop (b) Double-hop and (c) reffected-power configuration, redrawn from [43] 
Other group who was dedicated to study the through metal data transmission was at Drexel University. The target of the group was not for enclosed sensors but for repeating data across metal compartments of naval vessels. Some techniques described are the communication based on the echo-cancellation to suppress the channel reverberation [46], pulse amplitude modulation [47] and a scheme using the orthogonal frequency-division multiplexing (OFDM) [48-51-33]. In the investigated methods, they developed digital communication to improve data rate, from $50 \mathrm{kbps}$ to $30 \mathrm{Mbps}$. Other application that uses the acoustic waves was developed by Hosman et al. [52] based on multi-tone frequency-shiff keying (MFSK) to communicate with devices located inside a shipping container. The system was able to transmit low data at a rate of $360 \mathrm{bps}$.

Concurrently, research has focused on simultaneously wireless power and data transmission through metals layer. The Australian Defense Science Technology Organization developed and evaluated methods to deliver power and data transmission through metal plate for health monitoring sensors embedded within aircrafts. They presented an equivalent model based on Pspice that uses the analogy between the second order acoustic waves differential equations and the second order electrical transmission line equations [37,38]. The Pspice model implemented by Moss et al. in [37,38] was based on the thickness-mode transducer firstly presented by Leach [72] and subsequently extended by Püttmer et al. [73] to introduce a lossy transmission line considering transducers mechanical losses. Moss et al. also performed experiments which shown that $300 \mathrm{~mW}$ of power can be wireless transmitted through an aluminum plate with thickness in the range of $1.6 \mathrm{~mm}$ to $5 \mathrm{~mm}$ at an efficiency of $30 \%$ using a nominal transducer of $1 \mathrm{MHz}$ in thickness-mode vibration. Further publications also include power and data transmission simultaneously. In $[39,40]$ the described system is able to transmit $420 \mathrm{~mW}$ of power through an aluminum plate of $1.6 \mathrm{~mm}$ thick, representing a efficiency of $42 \%$, as well as a data rate of $115 \mathrm{kbps}$.

Aeronautic and aerospace applications helped to foster groups such as EADS Innovation Works, University of Paderborn, and Saarland University [53,54,55]. They have studied remote ultrasonic power and data transmission for sensors inside metal containers. One of the application uses a pair of thickness-mode transducer 
"sandwiched" attached to a $7 \mathrm{~mm}$ thick aluminum barrier, following the configuration of figure 2.6.

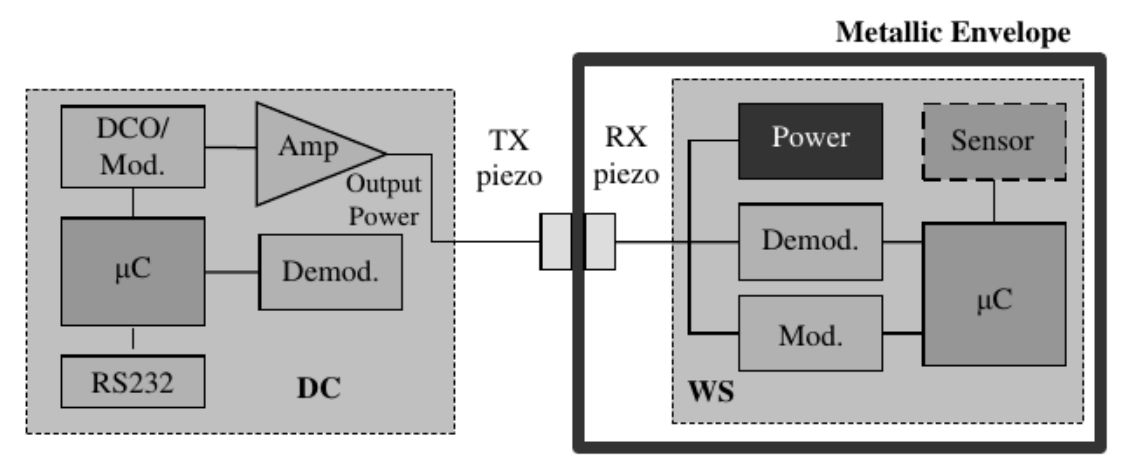

Figure 2.6 Power and data communication of a sensor inside metallic envelope, redrawn from [53]

The energy that is received in the wireless sensor (WS), which is isolated from the data concentrator (DC) by a metallic envelope supplies power to a digital processor and a digital sensor. The system has a halfduplex bidirectional communication, where in the WS-DC way the digital processor modulates the load coupled to the RX transducer. On the other hand, the DC-WC communication modulates the amplitude of the carrier signal. It has been demonstrated that the system can delivery $30 \mathrm{~mW}$ of power through the $7 \mathrm{~mm}$ thick aluminum layer and a data communication of $1 \mathrm{kbps}$ was achieved.

The research group at RPI, previously mentioned, has also extensively worked on the methods to simultaneously wireless transmit power and data through metal using acoustic waves. They addressed some important issues such as fullsystem prototypes evaluations [56,67], models of the acoustic-electric channel $[57,59,63,69]$, high-data-rate communication $[62,63,65]$, apparatus and methods to maximize the efficiency of power and data transfer $[60,63,64]$ and different types of configurations and methods [61,68,69] Shoudy et al. [56] developed a prototype based on the diagram of Fig. 2.4 in which the communication uses the "reffectedpower" with a pair of transducers operating at $1 \mathrm{MHz}$. The CW signal generated in the "outside" is transmitted to the "inside", where it is rectified and regulated to supply the sensors and peripheral circuitry. Additionaly, an electric switch varies the impedance of the inside transducer and consequently changes the amplitude of the $\mathrm{CW}$ signal. Experimental results have shown that the inside circuitry can harvest $250 \mathrm{~mW}$ of power, and reliable communication at rates of up to $55 \mathrm{kbps}$ with 57 
$\mathrm{mm}$ thick steel was achieved. In [67] the development of a prototype shows the acoustic power and data communication through a curved metal layer with $3.2 \mathrm{~mm}$ of thickness. The developed experiment has a low-power microcontroller located in the "inside" and operating passively. The author has also developed the boards of "inside" and "outside". The system shows the feasibility of transmitting data at $10 \mathrm{kbps}$ using amplitude modulation, with $0.02 \%$ of packet loss. Nevertheless, information on power consumption of the inside electronics was not provided and the sole component to be powered was a microcontroller.

The RPI group has been studying models to simulate the system based on Fig. 2.4. A finite element model of the system was used to characterize the impedance of the PZT transducer when attached on the metal layer, showing high correlation between the model and the experimental characterization [67]. In [68] many parameters concerning the power transfer of the ultrasonic channel were simulated, for instance, the transducer area, the metal wall thickness and compositions and the transducer-wall coupling effects and, strong agreement with experiments were obtained. Furthermore, Lawry et al [69] developed an analytical model based on $A B C D$ parameters for the sandwiched plate configuration of Fig. 2.4. The ABCD matrix represent the layers of the acoustic-electric channel, comprising the transducer and the intermediate layer, such as metals, couplants and fluids. The enhanced model shows great performance while reducing the complexity of finite element method. More recently, Wilt et al. [75] have developed a model that represent the layers of the acoustic-electric channel by a two-by-two travelling pressure wave transfer matrix related with the forward and reverse pressure waves on both faces of each layers. A comparison of the model with an experiment consisting of two co-aligned transducer and attached on stainless steel block, 74.8 $\mathrm{mm}$ thick, shows that the model is capable to reproduce the behavior of physical channels. It is also shown that the piezoelectric transducer model is compatible with the $\mathrm{ABCD}$ model, allowing the connection with electronic components.

The RPI group also explored different configurations to evaluate the behavior of acoustic power and data transer. Lawry et al. proposed [56-69] A novel configuration formed by two pairs of piezoelectric transducers, one of them operating at $1 \mathrm{MHz}$ with $66.7 \mathrm{~mm}$ of diameter and the other operating at $4 \mathrm{MHz}$ with $25.4 \mathrm{~mm}$ of diameter transmit power and data separately. The transducer with lower 
frequency transmits the power and the other, the data. A communication protocol based on OFDM turns the system into a high data-rate transmitter with a high spectral efficiency. The experiment reveals a communication data rate of 17.37 Mbps with $50 \mathrm{~W}$ of delivery power through a $63.5 \mathrm{~mm}$ thick steel. In [61], Lawry et al have built an experimental setup for high-temperature applications. The high temperature piezoelectric transducers were attached with a high temperature couplant and a clamp in order to operate inside a chamber with temperatures up to $260^{\circ} \mathrm{C}$. The method of data communication is based on "reffected-power". The results shown that the system is capable to transmit data at a rate of $50 \mathrm{kbps}$ from the inside to outside with $1 \mathrm{~W}$ of power transmission. A different configuration formed by a pair of coaxially aligned PZTs and a third PZT attached on the outside is simulated using an equivalent circuit based on Pspice® simulation [69]. In this publication an analysis of the power and data communication were performed with good agreement with experiment results. The RPI group also explored a multilayer channel that consists of a steel-water-steel [68] with $15.97 \mathrm{~mm}, 88.3 \mathrm{~mm}$ and 10.92 $\mathrm{mm}$ thick, respectively. The system was analyzed by S-parameters and tested experimentally showing that a data rate of $4 \mathrm{Mbps}$ and a power transfer efficiency of $30 \%$ is possible. This work is the only one that evaluates the power and data communication passing through metal and water multi-layers, showing an appeal to improve the studies that explores a multilayered configuration. Furthermore, the publication did not explore the feasibility of the system when exposed to high power or long periods of operating.

With a different approach, a group at Newcastle University utilized electromagnetic acoustic transducers (EMATs) to generate the acoustic waves inside the metal [5, 52, and 53]. They achieved $1 \mathrm{Mbps}$ of data rate through 25.4 $\mathrm{mm}$ thick metal with a transducer liffoff of $0.8 \mathrm{~mm}$ to the metal surface. However, the use of EMATs are very inefficient to transfer power due to its low electromechanical coupling. On the other hand, it has an extremely advantage of not requiring direct contact with the metal suppressing the use of couplants.

Comparing the methods to power and transmit data through a metal barrier it is possible to conclude that electromagnetic coupling are suitable for applications where the metal layer is thin and the data rate low. Ultrasonic waves otherwise have the ability to propagate inside metal with more efficiency and with high power and data transmission rates capabilities. However, historical review showed limited 
experiments that explore the development of prototypes with sensors and peripherals circuitry. Some challenges need to be solved for a proper engineering application. Studies about the long-term reliability and efficiency of the epoxies and coupling layers is one of the known drawbacks of the ultrasonic system based on PZT s transducers. Other possible problem is the inffuence of the temperature, pressure and other parameters in the system, rarely investigated. This survey also clarified that the choice of setup depends on the application as there is no optimum solution regarding both power and data communication. A specific application can be focused on achieving high data communication rates, or high power supply, or even concern a high temperature scenario. All of these issues require specific solution and all of them are in constant evolution. 


\section{3}

\section{Analytical and numerical design}

This section describes the overall system adopted in this work and two models used to simulate it; one based on Pspice electric circuit simulation and one analytical model, which has been implemented in MATLAB ${ }^{\circledR}$. Three major blocks are present in the system; the outside block, the inside block and the acoustic channel block, see Fig. 3.1. The outside block is composed by a signal generator, responsible to send a carrier signal, a PZT transducer, named outside transducer, and a circuit to demodulate the received signal. The inside block is composed by a circuit to rectify and store the received energy, a PZT transducer, named inside transducer, and a circuit to modulate the signal. The acoustic channel block is the material medium connecting both sides by means of ultrasonic wave propagating inside of it. The simplest conceivable channel consists of a metal barrier plate that separates both PZT transducer and the acoustic couplant that attach each PZT transducer on the metal plate, as shown in Fig. 3.1. Alternatively, the channel may be composed by several layer, instead of just a single metal block. For instance, there can be a metal layer and a liquid layer separating the transducers. These three blocks form the global system that is called power and data communication through acoustic channel, (PDAC).

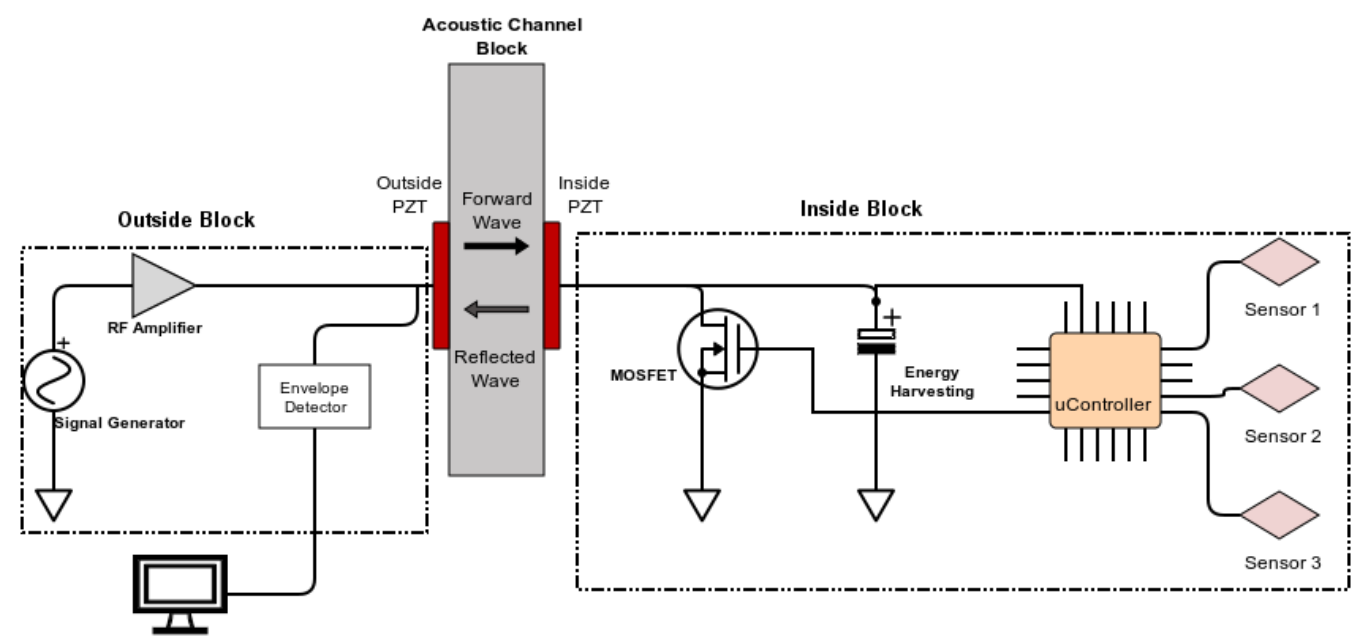

Figure 3.1 Overall diagram of PDAC generic system 
In the next sections the acoustic models implemented in PSPICE and MATLAB are explained. This two models are studied in order to understand the fequency and time domain behavior and characteristics of PDAC.

\section{1}

\section{Pspice modeling}

In order to simulate acoustic elements of the channel the approach proposed by Roa-Prada et al. [69] is adopted here. In this approach one considers analogies between the electric voltage and current and the sound pressure and velocity. In order to model the system, two different categories with different formulation are further described, the piezoelectric transducer and the intermediate layer.

\subsection{1}

\section{Piezoelectric transducer model}

The piezoelectric transducer can be modeled as a three port device with one electrical port and two mechanical ports. The electric signal is applied or sensed in the electrical port, whereas the two mechanical ports represent the front and back faces of transducer, which vibrate when mechanical waves are generated or sensed. As the analytical solution for this system is complex mainly due to the electromechanical analysis, an analogous model expressed in the electrical domain can be more practical and convenient. In this context, Leach [72] has built a model using transmission lines and controlled-source for piezoelectric transducer, Püttmer et al. [73] insert a lossy transmission line considering mechanical losses in the piezoelectric transducer and Deventer et al. [74] implemented a low loss approximation to model piezoelectric transducer. In this work the part of the model which specifically model the piezoelectric transducer is based on the thicknessmode transducer that Leach [72] has developed.

The diagram of the piezoelectric transducer illustrated in Fig. 3.2 shows two mechanical ports represented by force-velocity pair and one electrical port 
represented by voltage-current pair. Eq. (1)-(3) are the governing equations of this model. These are based on mechanical-electric relationship of a piezoelectric material.

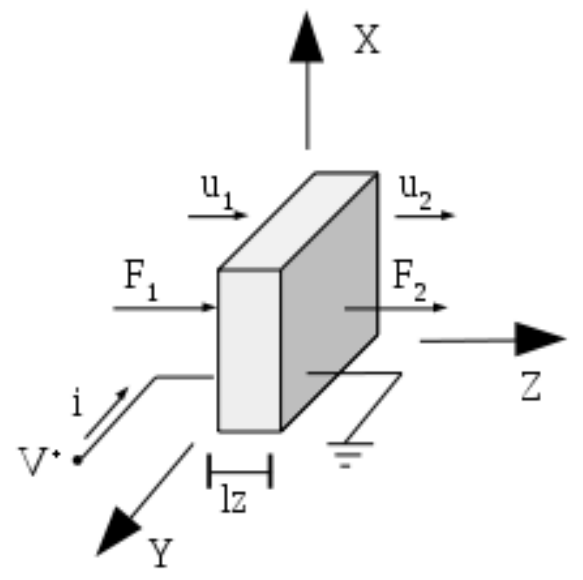

Figure 3.2 Diagram of the piezoelectric transducer

$$
\begin{gathered}
\frac{d f}{d z}=-\rho A s u \\
c \frac{d \zeta}{d z}=\frac{-1}{A} f+h D \\
E=-h \frac{d \zeta}{d z}+\frac{1}{\varepsilon} D
\end{gathered}
$$

$\rho$ is the density, A the transducer cross-sectional area, $\mathrm{c}$ is the relative elastic constant, whose common unit is $\left(\mathrm{N} / \mathrm{m}^{2}\right), \mathrm{h}$ is the piezoelectric constant whose common unit is $\left(\mathrm{Nm}^{4} / \mathrm{C}\right), \varepsilon$ is the permittivity whose common unit is $(\mathrm{F} / \mathrm{m})$, Eis the electric field intensity, $\mathrm{D}$ is the fux density, $\zeta$ is the particle displacement, $u$ is the particle velocity, $f$ is the free, $i$ is the current from the electrical signal and $z$ is the coordinate where the thickness of the transducer is distri buted.

Equations (1)-(3) are described in the frequency domain, in which an implicit Laplace Transform is considered. According to the Laplace Transform derivative and integration properties [76], a multiplication by " $\mathrm{s}$ " in the frequency domain 
represents a time differentiation in the time domain, whereas a division by " $\mathrm{s}$ " in the frequency domain means time integration in the time domain.

Being $i$ the current which flows in the external electrodes of transducer, $q$ the charge related by the equation $\mathrm{q}=\mathrm{I} / \mathrm{s}$ and the flux density $\mathrm{D}=\mathrm{I} /(\mathrm{sA})$, the equations (1) and (2) can be rewritten as:

$$
\begin{aligned}
& \frac{d}{d z}\left[f-\frac{h}{s} i\right]=-\rho \mathrm{A} s u \\
& \frac{d u}{d z}=-\frac{s}{\mathrm{Ac}}\left[f-\frac{h}{s} i\right]
\end{aligned}
$$

Drawing a parallel with telegraphist's equations [77] and manipulating the equations (1) and (2) one can obtain a relationship between transmission line and piezoelectric parameters. The telegraphist's equations are

$$
\begin{aligned}
& \frac{d V}{d z}=-L s I \\
& \frac{d I}{d z}=-C s V
\end{aligned}
$$

where the volt age $\mathrm{V}$ is analogous to $[\mathrm{f}-(\mathrm{h} / \mathrm{s}) \mathrm{i}]$ and the current $\mathrm{I}$ is analogous to the particle velocity $\mathrm{u}$. $\mathrm{L}$ and $\mathrm{C}$ are the distributed inductance and capacitance, respectively. The relationships between parameters of transmission line and the piezoelectric transducer equations are

$$
\begin{aligned}
& L=\rho \mathrm{A} \\
& C=\frac{1}{\mathrm{AC}}
\end{aligned}
$$

The phase velocity of the lossless transmission line is:

$$
u_{p}=1 / \sqrt{L C}
$$

Substituting the values of $\mathrm{L}$ and $\mathrm{C}$ in Eq. (9), the phase speed of the piezoelectric transducer is 


$$
u_{p}=\sqrt{(c / \rho)}
$$

Using the same principle for the characteristic impedance of a transmission line,

$$
Z_{0}=\sqrt{(L / C)}
$$

The characteristic impedance of the piezoelectric transducer can be obtained substituting the analogous values of L and C, from Eq. (8), and the expression of phase speed from Eq. (10), leading to

$$
Z_{0}=\rho \mathrm{A} u_{p}
$$

Making the capacitor per unit of length of Eq. (8.b) in function of the phase speed, from Eq. (10), instead of the relative elastic constant, imply that

$$
\mathrm{C}=1 /\left(\mathrm{A}_{\mathrm{z}} \rho \mathrm{u}_{\mathrm{p}}^{2}\right)
$$

The last analogy equation can be obtained integrating Eq. (3) from $z=0$ toz $=1 z$ and letting $\mathrm{D}=\mathrm{i} /(\mathrm{sA})$ and $\zeta=\mathrm{u} / \mathrm{s}$. The result is the vol tage across the transducer electrodes, which assumes the expression

$$
v=\frac{h}{s}[u 1-u 2]+\frac{1}{C_{0} s} i
$$

where $\mathrm{u} 1=\mathrm{u}(0), \mathrm{u} 2=\mathrm{u}(\mathrm{lz})$, and $\mathrm{C}_{0}$ is defined as

$$
\mathrm{C}_{0}=\varepsilon \mathrm{A} / \mathrm{lz}
$$

In order to build a circuit that can be designed in PSPICE, the transducer has to be modeled in two parts. The first one as a transmission line using the electrical and mechanical analogies and the relationship with the telegraphist's equation, and the second one by the electrical-mechanical coupling which is described by the Eq. (14).

As illustrated in Fig. 3.3(a)., T represents the transmission line, F1 and F2 the forces on the faces of transducer and $\mathrm{u} 1$ and $\mathrm{u} 2$ the particle velocities. In Fig. 3.3(b), there is a voltage $\mathrm{V}$ that is applied on the transducer and a capacitor $\mathrm{C} 0$, related to the area, thickness and dielectric of PZT transducer, according to Eq. (14).

There are two current-controlled voltage sources in Fig. 3.3. These sources represent the coupling between the mechanical and the electrical part. The 
mechanical part (Fig 3.3.a) has a voltage source controll ed by the injected current i. On the other hand, the electrical part (Fig. 3.3.b), presents the velocity difference (u1 - u2) as the "current"-controller.

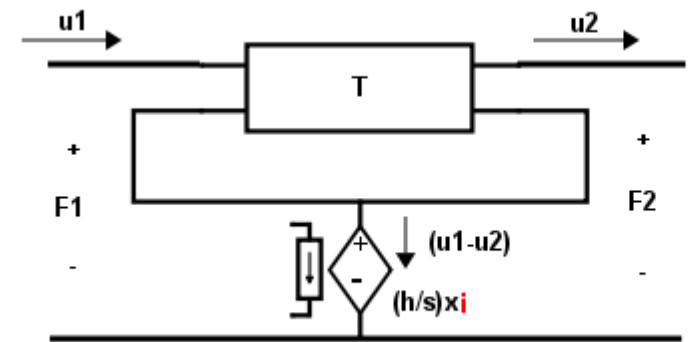

(a)

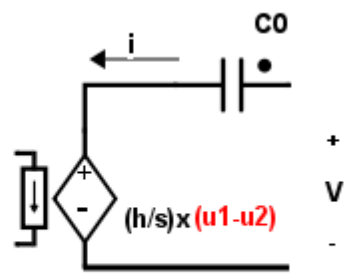

(b)

Figure 3.3 (a) Transmission line representing the mechanical part controlled by the current (i) and (b) the electrical part of the transducer controlled by the particle velocity

Having the design of the circuit, it is possible to implement it using an electrical simulation soffware. In this dissertation ORCAD 9.2 [88] was chosen since it has many PSPICE model and it is simple to manage. Fig 3.4 shows the analogous subcircuit for the thickness mode transducer created in ORCAD soffware which was obtained from the piezoelectric transducer from Fig. 3.3.

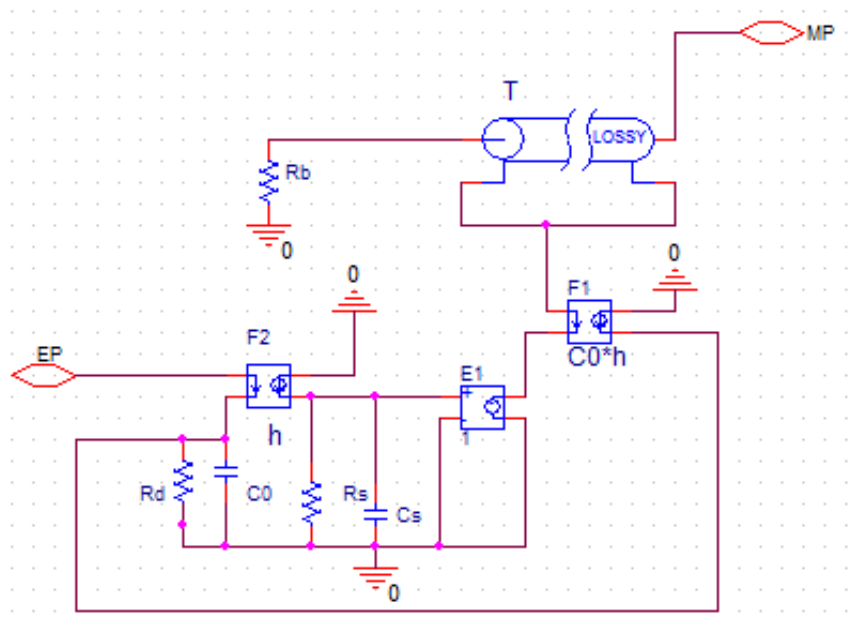

Figure 3.4 Transducer subcircuit schematic in ORCAD software

The electrical port (EP) in Fig. 3.4, represents the input/output voltage applied/sensed on the transducer. Connecting it with capacitor C0 and current controlled source F1, it has exactly the same function of the electrical part, illustrated in Fig. 3.3 (b). The reason to choose this current controlled source instead of voltage controlled source, is to avoid the use of complex variable s, since PSPICE 
does not have an element capable to treat it. This operation can be straightforward obtained by using the Norton-Thévenin equivalency [78]. The mechanical part, illustrated in Fig 3.3 (a) is added in the circuit of Fig. 3.4 by the parts: F2, Rs, $C_{S}$ and E1. In this context, it is possible to substitute the $1 / \mathrm{s}$ term in the transfer function of E1 by an integration operation, according to the Laplace Transform properties [76], which can be done by a capacitor, producing a electrical voltage

$$
V_{c}=\frac{1}{C_{s}} \int_{t} i d t
$$

For $\mathrm{C}_{\mathrm{s}}=1 \mathrm{~F}$, the dependent voltage source $\mathrm{E} 1$ is equal to $\mathrm{h}$ multiplied by the integral of the current injected in port EP. The resistor $\mathrm{R}_{\mathrm{S}}$ is included to prevent the terminal of $\mathrm{C}_{\mathrm{S}}$ to float, imposing a current path. For $\mathrm{R}_{\mathrm{S}}$ equals to $1 \mathrm{k} \Omega$, the $\mathrm{RC}$ time constant is several times greater than the transducer resonant frequency, not affecting then the time and frequency response. The front face of the transmission line, representing the second mechanical port (MP), is left opened to be connected to the next layer, which is the intermediate layer subcircuit. As the transmission line (T) from Fig. 3.4 represents the transducer, the length of the transmission line is equal to transducer thickness.

The back face of the transmission line is shorted with a resistor, $\mathrm{R}_{\mathrm{b}}$, that represents the mechanical impedance of the transducer back layer, in this case it is assumed to be air. Other resistor that appears in the circuit, $\mathrm{R}_{\mathrm{d}}$, is related to a complex dielectric loss. This other losses implemented in the model will be discussed in section 3.3. As one can see, a few number of elements are used to model the transducer. As acoustic impedance is associated with acoustic pressure, and the mechanical-electrical analogy relates force and voltage, and not pressure. The characteristic impedance of the mechanical layer has to be properly transformed in order to satisfy the relationship [3]. Equation (17) shows the relationship between force $(\mathrm{F})$ and pressure $(\mathrm{P})$.

$$
F=P A
$$

Analogously, the characteristic impedance can be rewritten multiplying the equation by the cross-section area as:

$$
Z=\rho u_{p} A
$$


Table 3.1 shows characteristics impedances from common materials used as mechanical layers [6].

Table 3.1 characteristic impedances of common materials

\begin{tabular}{|c|c|}
\hline Material & Z(MRayl) \\
\hline Air & $403 \times 10^{-3}$ \\
\hline Aluminum & 17.33 \\
\hline Steel & 45.7 \\
\hline Water $^{1}$ & 1.494 \\
\hline${\text { Completion } \text { fluid }^{1}}^{1}$ & $\sim 2.8$ \\
\hline
\end{tabular}

\section{1 .2}

\section{Intermediate layer model}

The intermediate layer can also be modeled by acoustic-electric analogies. Deventer [74] applied the approach of Püttmer [73] for liquids and solids to obtain an equivalent electrical circuit of acoustic waves propagation through materials. In this context, a comparison was made between electrical and mechanical domain al so using the telegraphist's differential Eq.(19) and the acoustic wave differential Eq. (20), respectively:

$$
\begin{aligned}
& \frac{d^{2} V(z)}{d z^{2}}-\gamma^{2} V(z)=0 \\
& \frac{d^{2} I(z)}{d z^{2}}-\gamma^{2} I(z)=0
\end{aligned}
$$

${ }^{1}$ Fluid use in wellbore after the drilling [100] 


$$
\begin{aligned}
& \frac{d^{2} P(z, t)}{d z^{2}}-k_{c}{ }^{2} P(z, t)=0 \\
& \frac{d^{2} u(z, t)}{d z^{2}}-k_{c}{ }^{2} u(z, t)=0
\end{aligned}
$$

where $\gamma$ is the propagation constant associated with the change of amplitude and phase of electromagnetic waves as it propagates in a material, and $\mathrm{k}_{\mathrm{c}}$ is acoustic the complex wave number, represented in Eq. (21) and (22), respectively.

$$
\begin{gathered}
\gamma=\sqrt{\left(R^{\prime}+j w L^{\prime}\right)\left(G^{\prime}+j w C^{\prime}\right)} \\
k_{c}=\frac{w}{u_{p}} \frac{1}{\sqrt{1+j w \tau}}
\end{gathered}
$$

where $\mathrm{R}^{\prime}, \mathrm{L}^{\prime}, \mathrm{G}^{\prime}$ and $\mathrm{C}^{\prime}$ are the elements of the transmission line per unit length, $\mathrm{w}$ is the angular frequency and $\tau$ is the relaxation time [3]. Using the low-loss condition where $w^{\prime}$ ' $>>R^{\prime}, w^{\prime}>>G^{\prime}$ and $1>>w \tau$, the Eq. (23) and (24) can be rewritten as:

$$
\begin{gathered}
\gamma=\alpha_{e}+i \beta=\frac{1}{2}\left(\frac{R^{\prime}}{L^{\prime}}+\frac{G^{\prime}}{C^{\prime}}\right) \sqrt{L^{\prime} C^{\prime}}+i w \sqrt{L^{\prime} C^{\prime}} \\
k_{c}=\alpha_{m}+i \kappa=\frac{w}{u_{p}} \frac{1}{\sqrt{2}}\left[\frac{\sqrt{1+(w \tau)^{2}}-1}{1+(w \tau)^{2}}\right]^{\frac{1}{2}}+i \frac{w}{u_{p}} \frac{1}{\sqrt{2}}\left[\frac{\sqrt{1+(w \tau)^{2}}+1}{1+(w \tau)^{2}}\right]^{\frac{1}{2}}
\end{gathered}
$$

With real part, $\alpha_{e}$, being an attenuation constant of the propagation constant and $\alpha_{\mathrm{m}}$ the attenuation of the complex wave number. The imaginary part, $\beta$ and $\kappa$, the phase constant and wave number, respectively.

Finally, the imaginaries parts are obtained applying the low loss approximation,

$$
\begin{gathered}
\beta=w \sqrt{L^{\prime} C^{\prime}} \\
\kappa=w / u_{p}
\end{gathered}
$$

Equaling $\beta$ and $\kappa$ gives the phase velocity of the intermediate layer in function of transmission line parameters L' and C'.

$$
u_{p}=\frac{1}{\sqrt{L^{\prime} C^{\prime}}}
$$

Using another analogy relationship, this one between the electrical and mechanical characteristic impedance we can assign:

$$
Z_{e l}=\sqrt{\frac{R^{\prime}+j w L^{\prime}}{G^{\prime}+j w C^{\prime}}}
$$


And for the lossy acoustic medium, $\mathrm{Z}_{\mathrm{ac}}[6]$ :

$$
Z_{a c}=\rho u_{p} \sqrt{1+j w \tau}
$$

where the subscripts el and ac stand for electric and acoustic, respectively. Considering a low-loss approximation, the electrical and mechanical characteristics impedances become:

$$
\begin{aligned}
& Z_{e l}=\sqrt{\frac{L^{\prime}}{C^{\prime}}} \\
& Z_{a c}=\rho u_{p}
\end{aligned}
$$

Using the same principle from transducer model in which the force, and not pressure, is represented by voltage. The equivalence between the two characteristic impedance is simply

$$
Z_{e l}=Z_{a c} \mathrm{~A}
$$

Using the equations (26), (27), (28) and the relationship equation (31), one can assign the following relationships:

$$
\begin{gathered}
L^{\prime}=\mathrm{A} \rho \\
C^{\prime}=\frac{1}{\mathrm{~A} \rho u_{p}{ }^{2}}
\end{gathered}
$$

With Eq. (8) to (33), one can model in PSPICE an equivalent circuit of the piezoelectric transducer, as well as, an intermediate layer. The transducer may be of any piezoelectric material, whereas the intermediate layer can assume any acoustic material, either a metal or fluid, or even a cascade combination of many different layers. The circuit analogies between electric and acoustic elements are summarized in Table 3.2 for the piezoelectric transducer and in Table 3.3 for the intermediate layer.

Table 3.2 Analogies between mechanical and electrical of PZT transducer

\begin{tabular}{|c|c|}
\hline Electrical & Mechanical \\
\hline $\mathrm{V}$ & {$[\mathrm{f}-(\mathrm{h} / \mathrm{s}) \mathrm{i}]$} \\
\hline $\mathrm{I}$ & $\mathrm{u}$ \\
\hline $\mathrm{L}^{\prime}$ & $\rho \mathrm{A}_{\mathrm{z}}$ \\
\hline $\mathrm{C}^{\prime}$ & $1 /\left(\mathrm{A}_{\mathrm{z}} \rho \mathrm{u}_{\mathrm{p}}{ }^{2}\right)$ \\
\hline
\end{tabular}


Table 3.3 Analogies between mechanical and electrical of intermediate layers

\begin{tabular}{|c|c|}
\hline Electrical & Mechanical \\
\hline $\mathrm{V}$ & $\mathrm{f}$ \\
\hline $\mathrm{I}$ & $\mathrm{u}$ \\
\hline $\mathrm{L}^{\prime}$ & $\rho \mathrm{A}_{\mathrm{z}}$ \\
\hline $\mathrm{C}^{\prime}$ & $1 /\left(\mathrm{A}_{z} \rho \mathrm{uu}_{\mathrm{p}}{ }^{2}\right)$ \\
\hline
\end{tabular}

The model of piezoelectric transducer is straightforward obtained if all parameters are known, however this task could be sometimes challenging. The intermediate layer, on the other hand, models all the layer that are not piezoelectric as a transmission line. Acoustic propagation in solids and fluids can be modeled using this approach, considering that the relevant wave propagation occurs in one direction by a single acoustic wave mode.

\subsection{3}

\section{Pspice loss considerations}

Up to here, the model presents the electrical parameters that have the equivalence to the acoustic equations, however for a more realistic model a loss considerations is introduced in this section. As demonstrated by Deventer [74] and Püttmer [73], a lossy transmission line is used to model transducers and intermediate layers introducing a resistive element in both transmission lines, which represents the loss factor of the transducer and intermediate layer. This resistance can be calculated using the real part of the complex propagation constant $\gamma$, which is the attenuation coefficient $\alpha$ of the transmission line. With a low loss approximation one assumes that $\mathrm{G}^{\prime}=0$ and $w L^{\prime}>>R^{\prime}$ and the complex propagation constant given in Eq. (23), can be divided in two different approach, one concerning the piezoelectric transducer and other the intermediate layer.

a) Piezoelectric transducer

Considering a low loss approximation, the complex propagation constant of the transmission line can be approximated by the low order terms of Taylor series 
expansion of (25). The real part, which is the attenuation coefficient, is then simplified to

$$
\alpha \approx \frac{R^{\prime}}{2 u_{p} L^{\prime}}
$$

Knowing that in a series RLC circuit, the quality factor,( Q, which represents the ratio of the energy stored to the energy dissipated, is described as:

$$
Q=\frac{w L^{\prime}}{R^{\prime}}=\frac{1}{\delta_{巾}}
$$

where $\delta_{\varphi}$ is the mechanical loss factor of the piezoelectric transducer. Most manufactures provide the mechanical loss factor $\delta_{\varphi}$ in their material datasheet as the inverse of the mechanical quality factor $\mathrm{Q}$.

Therefore, the attenuation coefficient can be rewritten as

$$
\alpha \approx \frac{w}{2 u_{p}} \cdot \frac{R^{\prime}}{w L^{\prime}}=\frac{w}{2 u_{p}} \delta_{\uparrow}
$$

Note that one can write the $\mathrm{R}$ ' parameter as:

$$
R^{\prime}=\frac{W L^{\prime}}{Q}
$$

Other loss that impacts the performance of the transducer is the dielectric loss. This loss can be explained since the capacitance $\mathrm{C}_{0}$ has a complex dielectric loss, which can be modeled using a resistor, $\mathrm{R}_{\mathrm{d}}$, in parallel with $\mathrm{C}_{0}$, with value:

$$
R_{d}=\frac{1}{\omega \delta_{e} C_{0}}
$$

It is worth highlighting that PSPICE simulators allow only constant values for $\mathrm{R}$, therefore an approximate value can be obtained using $\omega$ fixed and equals to the resonance frequency of the piezoelectric transducer.

\section{b) Intermediate layer}

The intermediate layer loss, is a combination of viscous losses and diffaction losses. Using the same approach from piezoelectric transducer loss, the viscous loss part of $\mathrm{R}^{\prime}$ can be determined making the same low loss approximation, however using the relationship with attenuation coefficient instead of loss factor. . The R' 
from the transmission line is obtained from Eq. (34), substituting $L^{\prime}$ and $C^{\prime}$ by its mechanical equivalence:

$$
R^{\prime}=2 \rho u_{p} A \alpha_{v}
$$

the coefficient $\alpha_{v}$ account for the attenuation due to viscous losses in $(\mathrm{Np} / \mathrm{m})$.

The diffaction part of $R^{\prime}$ represents a phenomenon that occurs inside a thick material layer, when the piezoelectric transducer area is smaller than the surface area of intermediate layer [79]. This loss can be understood as an amount of ultrasonic energy that spreads from the center axis that separates both transducers as illustrated in Fig. 3.5..

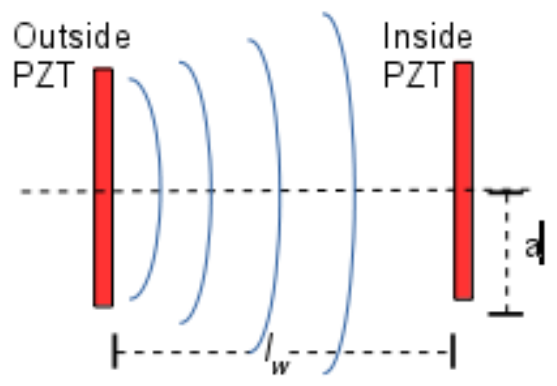

Figure 3.5 Spreading attenuation of acoustic wave

Bass [80] formulated an expression for the ratio of the average energy on the receiver transducer to the average energy at the transmitter transducer. The spreading attenuation is expressed as:

$$
\alpha_{s}(d B)=10 \log \left(\frac{p_{r}{ }^{2}}{p_{t}^{2}}\right)
$$

Where $p_{r}$ and $p_{t}$ stand for the intensity pressure received on the receiver transducer and the intensity pressure transmitted by the transmitter transducer. The ratio of their squares is approximately equal to

$$
\frac{p_{r}{ }^{2}}{p_{t}{ }^{2}} \simeq 1-2(\pi \xi)^{-\frac{1}{2}}\left(1-\frac{\xi^{2}}{2 \beta^{2} a^{2}}\right)+2(\pi \xi)^{-1}\left(1-\frac{\xi^{2}}{2 \beta^{2} a^{2}}\right)^{2}
$$

the dummy variable $\xi$ is defined as:

$$
\xi=\left(\frac{\beta}{2}\right)\left[\left(l^{2}{ }_{w}+4 a^{2}\right)^{\frac{1}{2}}-l_{w}\right]
$$


The attenuation $\alpha_{s}$ can be expressed in $\mathrm{dB} / \mathrm{m}$ or $\mathrm{Np} / \mathrm{m}$, by knowing that one Neper is equal to $8.6858 \mathrm{~dB}$. The effective attenuation is a sum of the viscous loss and the spreading loss. The quantity of spreading attenuation can be incorporated in the R' element of transmission line, being the total loss coefficient equal to:

$$
\alpha_{\text {total }}=\alpha_{v}+\alpha_{s}
$$

With this three types of losses we can implement the model of the transducer and intermediate layer in PSPICE with proper accuracy. The introduced losses are however low loss approximation models. In the next sections an explanation of the overall system used is described and some simulations of this system are performed.

\subsection{3}

\section{Full system model}

Gathering the piezoelectric transducer and the intermediate model, it is possible to build an equivalent circuit that covers all the mechanical and electrical parts. In Fig. 3.6 a full system is designed using ORCAD soffware, with transducer blocks representing the transducer model and the metal and adhesive transmission lines representing the intermediate layer.

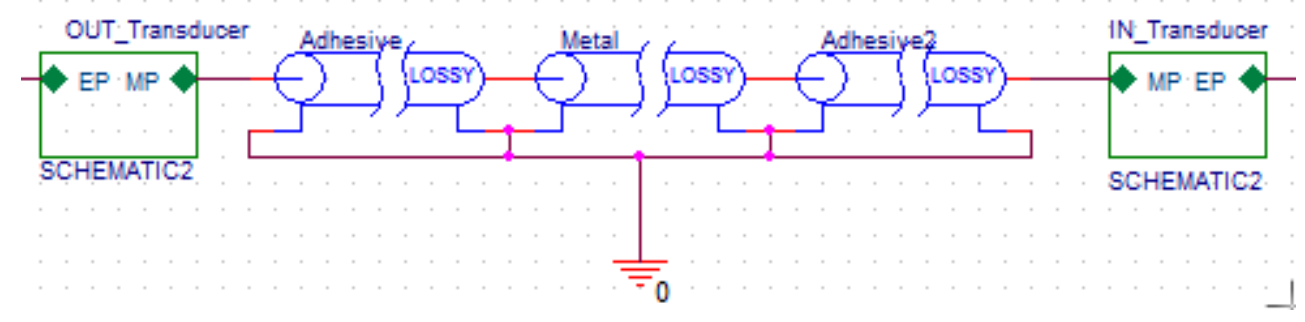

Figure 3.6 Full system circuit designed in ORCAD

Parameters used to build the transducer and intermediate layer are shown in Table 3.4. These values of piezoelectric transducer are obtained from PI Ceramic material [81] data sheet and complemented with piezoelectric ceramic equations of characterization [84]. The intermediate layers properties from adhesive 
(Huntsman ${ }^{\circledR}$ Araldite 2015) and aluminum are obtained using materials datasheet[82] and literature reference[83].

Table 3.4 Materials properties

\begin{tabular}{|c|c|}
\hline Transducer Properties & Value \\
\hline At $\left(\mathrm{mm}^{2}\right)$ & 400 \\
\hline$\varepsilon 33(\mathrm{t}) / \varepsilon 0$ & 1200 \\
\hline $\mathrm{k}_{33}$ & 0.66 \\
\hline $\mathrm{k}_{\mathrm{t}}$ & 0.46 \\
\hline$\rho_{\mathrm{t}}\left(\mathrm{Kg} / \mathrm{m}^{3}\right)$ & 7800 \\
\hline $\mathrm{Q}$ & 2000 \\
\hline $\mathrm{c} 33(\mathrm{~d})\left(\mathrm{N} / \mathrm{m}^{2}\right)$ & $16.6 \mathrm{E} 10$ \\
\hline $\tan _{\mathrm{d}}$ & $3 \times 10^{-3}$ \\
\hline
\end{tabular}

\begin{tabular}{|c|c|}
\hline Adhesive Properties & Value \\
\hline $\operatorname{At}\left(\mathrm{mm}^{2}\right)$ & 400 \\
\hline$\rho_{\mathrm{a}}\left(\mathrm{Kg} / \mathrm{m}^{3}\right)$ & 1400 \\
\hline $\mathrm{va}(\mathrm{m} / \mathrm{s})$ & 2100 \\
\hline$\alpha(\mathrm{dB} / \mathrm{m})$ & 1500 \\
\hline
\end{tabular}

\begin{tabular}{|c|c|}
\hline Metal Properties & Value \\
\hline $\operatorname{At}\left(\mathrm{mm}^{2}\right)$ & 400 \\
\hline$\rho_{\mathrm{m}}\left(\mathrm{Kg} / \mathrm{m}^{3}\right)$ & 2700 \\
\hline $\mathrm{vm}(\mathrm{m} / \mathrm{s})$ & 6420 \\
\hline$\alpha(\mathrm{dB} / \mathrm{m})$ & 2 \\
& \\
\hline
\end{tabular}




\subsection{4}

\section{Analysis of power transfer}

Analyze how the energy pass through the metal is one of the essential scopes of this dissertation. As one can see in Fig. 3.7, the implemented system is represented by two transducer blocks and three transmission lines. A frequency domain analysis using S-parameters [85] is then performed to study the characteristics of power transfer between transducers.

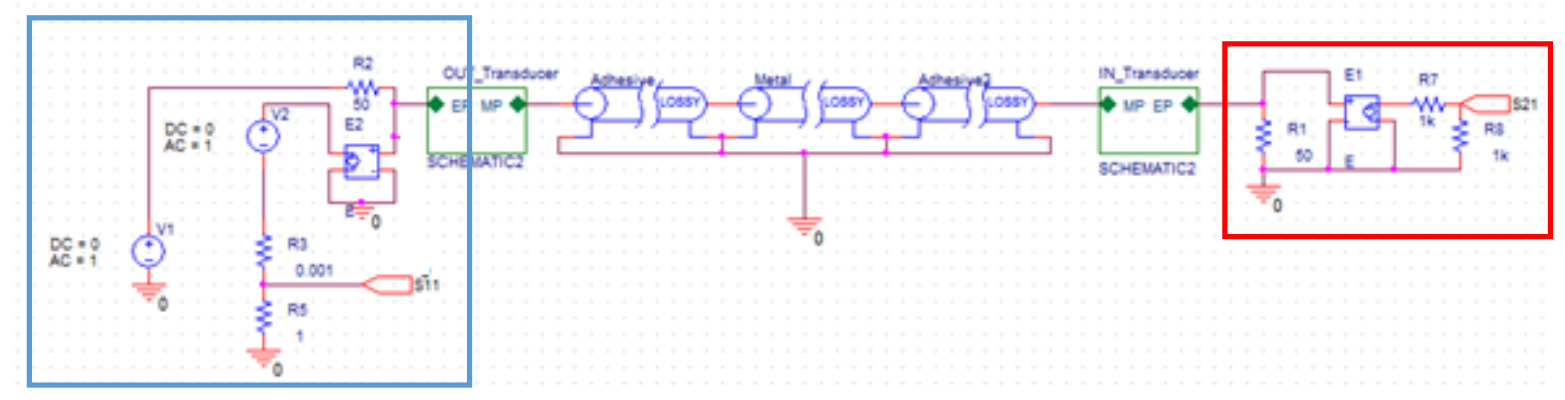

Figure 3.7 Acoustic channel with S11 and S21 blocks represented in blue and red, respectively

Scattering parameters, which are commonly referred to as S-parameters, is related to the traveling waves that are emitted or reffected in a network withn-ports. In other words, this means that with S-parameters we can measure the quantity of power that is incident/reffected in one port and reffected/incident from the same port or from another port. Figure 3.8 illustrates this system containing two ports.

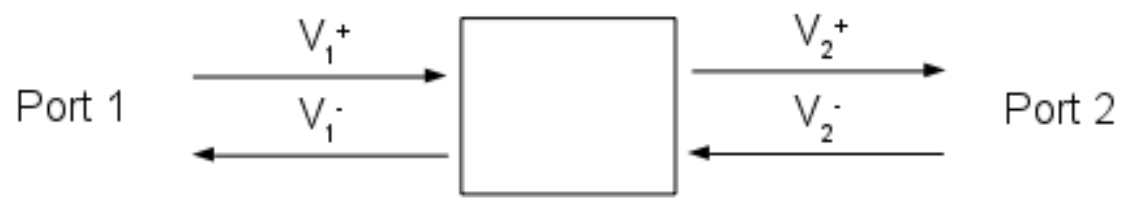

Figure 3.8 Two-port configuration of S-parameters

Therefore, we can define the individual S-parameter coefficients S11.S12, S21 and S22 as a function of the incident and reflected waves, according to Table 3.5.2. 
Table 3.5 2-port S-parameters

\begin{tabular}{|l|l|}
\hline $\mathrm{S} 11$ & $\mathrm{~V}_{1}^{-} / \mathrm{V}_{1}^{+}$ \\
\hline $\mathrm{S} 12$ & $\mathrm{~V}_{1}^{-} / \mathrm{V}_{2}^{+}$ \\
\hline $\mathrm{S} 21$ & $\mathrm{~V}_{2}{ }^{-} / \mathrm{V}_{1}^{+}$ \\
\hline $\mathrm{S} 22$ & $\mathrm{~V}_{2}^{-} / \mathrm{V}_{2}^{+}$ \\
\hline
\end{tabular}

To transform these voltage coefficients into power coefficients, there is a commonly used expression that relates the gain in $\mathrm{V} / \mathrm{V}$ to $\mathrm{dB}$ :

$$
g=20 \log _{10}\left|S_{x x}\right|
$$

To perform the analysis in ORCAD, a linear AC sweep analysis with frequency range from 1 to $4 \mathrm{MHz}$ containing 1601 points is generated [86]. With S21 and S11, one can extract some characteristics of power transfer and reffections of acoustic waves, respectively. In order to obtain these values in SPICE one can connect to the input and output terminals ofa two-port components specific circuits specially designed for this purpose, as proposed in [99]. These circuits are shown in Fig. 3.7, the left one highlighted in blue obtains the S11 and the right highlight ed in red the $\mathrm{S} 21$.

\subsection{5}

\section{Analysis of Data Communication}

Other fundamental study, is a time domain analysis. This type of analysis is used in order to investigate how data communication develops. As we switch the terminal load of the output PZT transducer between two values, a change in the input impedance seen by the generator is obtained, leading to an increase or decrease of the reffected waves. In the generator side this can be interpreted as a variation of the signal's amplitude or, as an amplitude modulation (AM).

The PZT transducer has a mechanical characteristic impedance that depends of stiffiness $\left(\mathrm{C}_{33}\right)$ and the phase velocity $\left(\mathrm{u}_{\mathrm{p}}\right)$, expressed in Eq. (30). The stiffness, otherwise known as elastic constant, can assume two distinct values represented by 
the parameter $\left(\mathrm{C}_{33}^{\mathrm{D}}\right)$ and $\left(\mathrm{C}_{33}^{\mathrm{E}}\right)$. The difference between them is exactly what can be used to perform amplitude modulation, as the parameter $\left(\mathrm{C}_{33}^{\mathrm{D}}\right)$ refers to a constant elastic when the electrodes are open-circuited and the parameter $\left(\mathrm{C}^{\mathrm{E}}{ }_{33}\right)$ refers to a constant elastic when the electrodes are short-circuited [87]. Accordingly to this, two resistance element can be connected in parallel to the electrodes of the PZT transducer to act as an open-circuited or a short-circuited.

A transient analysis in Pspice shows a curve in time of the amplitude modulation on the outside transducer. To realize this simulation in Pspice, a circuit called modulation block is built. The modulation block represented in Fig. 3.9 switches the terminal load of the output transducer into two distinct values, in this case between $\mathrm{R} 1=50 \Omega$ and $\mathrm{R} 2=0.22 \Omega$. These values are chosen to simulate a load when the inside PZT are providing power to the inside block and when the inside PZT is short-circuited with a MOSFET transistor with $0.22 \Omega$ of Rds(on).

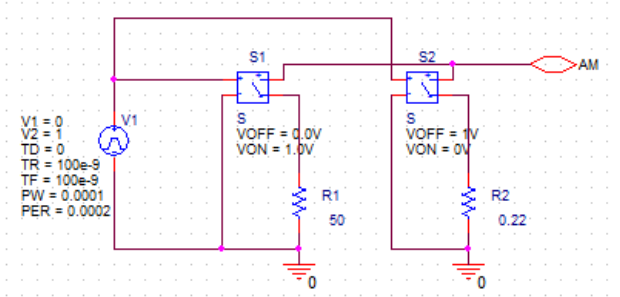

Figure 3.9 Modulation block

\section{2}

\section{Analytical modeling}

An analytical analysis of the same system described in the previous section can be implemented with technical computer soffware. Lawry [63] has designed an analytical model of a co-axially aligned piezoelectric transducer using the transfer matrix method with mixed-domain two-port ABCD matrices.

The same parameters for the both transducers, adhesive layer, metal layer and transducer backing layer used in Pspice are used in the analytical model, which was implemented using MATLAB $\AA$. This model is more realistic because it has both frequency dependent parameters and complex coefficient, which cannot be used in PSPICE. 


\subsection{1}

Two-port $A B C D$ Matrix

One form of representing the piezoelectric transducer and the intermediate layer is to use cascaded two-port ABCD matrices. The acoustic channel can be modeled by a series multiplication ofthe different types of ABCD matrix, in which each matrix represent a layer of the channel. Lawry [63] proposed this type of transfer matrix to model the electro-mechanical, purely mechanical and purely electrical layers using the analogy between electrical voltage(V) and mechanical force $(\mathrm{F})$ and between mechanical particle velocity(u) and electrical current (I). Therefore, there are four kinds of ABCD matrix of interest for modeling the whole acoustic channel, given by

$$
\begin{aligned}
& \left(\begin{array}{l}
F_{1} \\
u_{1}
\end{array}\right)=\left(\begin{array}{ll}
A_{m m} & B_{m m} \\
C_{m m} & D_{m m}
\end{array}\right)\left(\begin{array}{l}
F_{2} \\
u_{2}
\end{array}\right) \\
& \left(\begin{array}{l}
V_{1} \\
I_{1}
\end{array}\right)=\left(\begin{array}{ll}
A_{e m} & B_{e m} \\
C_{e m} & D_{e m}
\end{array}\right)\left(\begin{array}{l}
F_{2} \\
u_{2}
\end{array}\right) \\
& \left(\begin{array}{l}
F_{1} \\
u_{1}
\end{array}\right)=\left(\begin{array}{ll}
A_{m e} & B_{m e} \\
C_{m e} & D_{m e}
\end{array}\right)\left(\begin{array}{l}
V_{2} \\
I_{2}
\end{array}\right) \\
& \left(\begin{array}{l}
V_{1} \\
I_{1}
\end{array}\right)=\left(\begin{array}{ll}
A_{e e} & B_{e e} \\
C_{e e} & D_{e e}
\end{array}\right)\left(\begin{array}{l}
V_{2} \\
I_{2}
\end{array}\right)
\end{aligned}
$$

The subscripts (m) and (e) specify which type of ABCD matrix it is representing, in which $\mathrm{m}$ stands for mechanical and e for electrical. Thus the possible matrices represent a layer in which its ports are of following kinds: mechanical-mechanical ports, electrical-mechanical ports, mechanical-electrical ports or electrical-electrical ports. Therefore, it is possible to represent all sub systems of the channel by a two port $\mathrm{ABCD}$ matrix. 


\section{2 .2}

\section{Piezoelectric transducer model}

Royer [92] introduced the concept of impedance matrix, considering a onedimensional model of a bulk piezoelectric. The analytical model uses the same approach based on the wave equation as the one adopted in PSPICE for treating the transducer. Therefore, it has the same number of ports to represent the transducer as the previous presented model, i.e., two mechanical and one electrical. Fig. 3.10 illustrates the cross section of piezoelectric plate transducers. In which an electric voltage is injected in electric port generating mechanical waves that propagate inside the transducer. The model only predicts one-dimensional propagation, in this case, in $z$ direction. On each boundary of the transducer there is a mechanical port represented by the particle velocity and the force. Connecting the transducers mechanical port to an adjacent mechanical port can produce forward and backward waves, illustrated in Fig. 10, ifthe materials have distinct characteristic impedances.

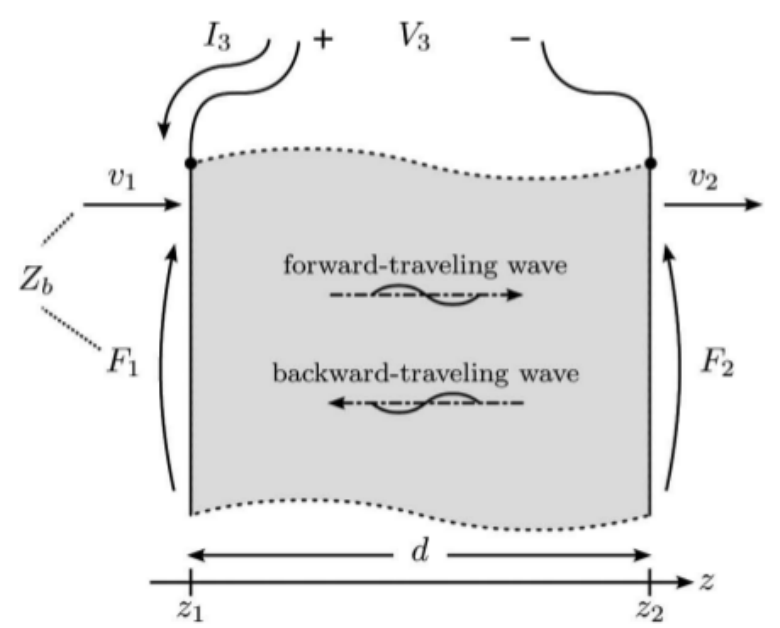

Figure 3.10 Piezoelectric mechanical and electrical ports, redrawn from [63]

The mechanical ports are governed by the following equation:

$$
F=-A_{z} T(z)=Z\left(a e^{-i k_{c} z}-b e^{i k_{c} z}\right)-i \frac{h}{w} I
$$

where $\mathrm{Z}$ corresponds to the characteristic impedance multi pl ied by the cross section area.

Expressing this equation in terms of velocities at both fices, implies: 


$$
\begin{aligned}
& F_{1}=\mathrm{Z}\left(\frac{u_{1}}{i \tan k d}+\frac{u_{2}}{i \sin k d}\right)+\frac{h I}{i \omega} \\
& F_{2}=\mathrm{Z}\left(\frac{u_{1}}{i \sin k d}+\frac{u_{2}}{i \tan k d}\right)+\frac{h I}{i \omega}
\end{aligned}
$$

This two forces are the forces at the two mechanical ports of the piezoelectric transducer. The other port is the electrical port. In this port the voltage applied between the faces of transducer can be calculated as the integral of the electric field E (Eq. 3) and is expressed below:

$$
V=\int_{z 1}^{z 2} E d z
$$

Where $(\mathrm{z} 2-\mathrm{zl})$ is the thickness of the transducer.

The impedance matrix for the pi ezoel ectric port assumes the expression:

$$
\left(\begin{array}{c}
F_{1} \\
F_{2} \\
V
\end{array}\right)=-i\left(\begin{array}{ccc}
\mathrm{Z} / \tan k d & \mathrm{Z} / \sin k d & h_{33} / \omega \\
\mathrm{Z} / \sin k d & \mathrm{Z} / \tan k d & h_{33} / \omega \\
h_{33} / \omega & h_{33} / \omega & 1 / \omega C_{0}
\end{array}\right)\left(\begin{array}{c}
u_{1} \\
u_{2} \\
I
\end{array}\right)
$$

To accomplish the analytical analysis using ABCD matrix, it is necessary to transform the impedance matrix of three ports into one of two ports in order to be used as an electrical-to-mechanical 2x2 ABCD matrix. Since one of the mechanical ports has a termination load, $\mathrm{Z}_{\mathrm{b}}$, them the load constrains a relationship between the voltage and current of that port. Consequently, one degree of freedom is lost. The $2 \times 2$ matrix of Eq. (54) is obtained from algebraic manipulation of Eq. (53)

$$
\left(\begin{array}{l}
V_{1} \\
I_{1}
\end{array}\right)=\left(\begin{array}{ll}
A_{e m} & B_{e m} \\
C_{e m} & D_{e m}
\end{array}\right)\left(\begin{array}{l}
F_{2} \\
u_{2}
\end{array}\right)
$$

Where: 


$$
\begin{aligned}
A & =-\frac{1}{h_{33} C_{0}} \frac{Z+Z_{b} \tanh (\Upsilon d)}{Z[\operatorname{sech}(\Upsilon d)-1]-Z_{b} \tanh (\Upsilon d)} \\
& -j \frac{h_{33}}{w Z} \frac{Z \tanh (\Upsilon d)}{Z[\operatorname{sech}(\Upsilon d)-1]-Z_{b} \tanh (\Upsilon d)} \\
B & =-\frac{Z}{h_{33} C_{0}} \frac{Z \tanh (\Upsilon d)+Z_{b}}{Z[\operatorname{sech}(\Upsilon d)-1]-Z_{b} \tanh (\Upsilon d)} \\
& +j \frac{h_{33}}{w Z} \frac{2}{Z[\operatorname{sech}(\Upsilon d)-1]-Z_{b} \tanh (\Upsilon d)} \\
C & =-j \frac{w}{h_{33}} \frac{Z+Z_{b} \tanh (\Upsilon d)}{Z[\operatorname{sech}(\Upsilon d)-1]-Z_{b} \tanh (\Upsilon d)} \\
D & =-j \frac{w Z}{h_{33}} \frac{Z Z_{b} \tanh (\Upsilon d)}{Z[\operatorname{sech}(\Upsilon d)-1]-Z_{b} \tanh (\Upsilon d)}
\end{aligned}
$$

where $\Upsilon$ and $\mathrm{C}_{0}$ where previously defined in Eq. (21) and (15), respectively, and w, $\mathrm{h}_{33}$ and dwhere previously defined in section 3.1.1

The constant $Z$, that is the modified characteristic acoustic impedance, which is given by

$$
Z=A \rho u_{p}\left(1-j \frac{\boldsymbol{\alpha}}{\beta}\right)
$$

Where $u_{p}, \rho$, and A were previously defined in section 3.1.1. One can notice that in Eq. (59) the constant (A) refers to a cross section area that is not related to the area (A) of Eq. (55). Another constant that appears in the matrix parameters is $Z_{b}$, illustrated in Fig. 3.10, which represent the acoustic impedance in the back face of the piezoelectric model.

One can extend this approach by considering that the acoustic channel has two piezoelectric, one transmitting and other receiving. The model described above shows the details of the transmitter model. For the receiving piezoelectric model, Lawry [63] demonstrated that the model is defined by the ABCD mechanicalelectrical matrix below:

$$
\left(\begin{array}{l}
F_{1} \\
u_{1}
\end{array}\right)=\left(\begin{array}{ll}
A^{\prime} & B^{\prime} \\
C^{\prime} & D^{\prime}
\end{array}\right)\left(\begin{array}{l}
V_{2} \\
I_{2}
\end{array}\right)
$$


The elements of the receiver matrix has the following relationship with the ones of the transmitting piezoelectric matrix:

$$
\left(\begin{array}{ll}
A^{\prime} & B^{\prime} \\
C^{\prime} & D^{\prime}
\end{array}\right)=\left(\begin{array}{ll}
D & B \\
C & A
\end{array}\right)
$$

\subsection{3}

\section{Intermediate layer model}

As the intermediate layer has no electrical port the ABCD matrix ofthis layer has the mechanical-mechanical configuration. To model the intermediate layer, Lawry [63] used the one-dimensional wave equation for particle displacement $\delta$ along the $\mathrm{z}$ direction:

$$
\frac{d^{2} \delta}{d z^{2}}=\frac{1}{u_{p}} \frac{d^{2} \delta}{d t^{2}}
$$

A solution for this equation is a spatial and temporal harmonic oscillation for the particle displacement:

$$
\delta(z)=\left(a e^{-r z}+b e^{r z}\right) e^{j w t}
$$

By deriving equation (63) one can apply the result equation at $z_{1}$ and $z_{2}$, as illustrated in Fig. 3.10, that yield the following expressions for the particle velocities $^{2}$. Both equations suppress the time oscillation term :

$$
\begin{aligned}
& u_{1}=u\left(z_{1}\right)=j w\left(a e^{-\gamma z_{1}}+b e^{\gamma z_{1}}\right) \\
& u_{2}=u\left(z_{2}\right)=j w\left(a e^{-\gamma z_{2}}+b e^{y z_{2}}\right)
\end{aligned}
$$

Manipulating the Eq. (64) and (65), considering that $d$ is equal to $\mathrm{z}_{2}-\mathrm{z}_{1}$, and solving for $a$ and $b$, yields:

${ }^{2}$ Figure 3.10 represent the velocities with (v) variable, however to keep the notation of this thesis, the velocities are represented by $(u)$ variable. 


$$
\begin{aligned}
& a=\frac{u_{1} e^{\Upsilon z_{2}}-u_{2} e^{\Upsilon z_{1}}}{j 2 w \sinh (\Upsilon d)} \\
& b=\frac{u_{2} e^{-\Upsilon z_{1}}-u_{1} e^{-\Upsilon z_{2}}}{j 2 w \sinh (\Upsilon d)}
\end{aligned}
$$

The relationship between force acting in both ports of the layer and the particle velocity starts from the Hooke's law [93]

$$
\sigma=E \varepsilon
$$

where $\sigma$ is the stress tensor and $\varepsilon$ the strain tensor, and $E$ is the stiffiness tensor.

Considering a unidirectional displacement along $\mathrm{z}$ direction the strain tensor is only nonzero at the normal $\mathrm{z}$ direction, this component is thus equal to:

$$
\varepsilon=\frac{d u}{d z}
$$

The same principle applies for considering the stress sensor presenting only one component, which is the proportional to the force according to Eq (17). Applying (69) and (17) into (68) leads to

$$
F=E A \frac{d u}{d z}
$$

Expanding Eq. (70) one can express the force at any position $z$ along the layer, which assumes the form (suppressing time-variation) of

$$
F(z)=-A E \Upsilon\left(a e^{-\Upsilon z}-b e^{\Upsilon z}\right)
$$

Eq. (71) can be written in function of the specific acoustic impedance (Z) as

$$
F(z)=-j w Z\left(a e^{-\Upsilon z}-b e^{\Upsilon z}\right)
$$

Applying equations (66) and (67) in (72) and further rearranging the obtained expressions to satisfy the ABCD configuration, one finally obtains the mechanicalmechanical ABCD matrix as:

$$
\left(\begin{array}{l}
F_{1} \\
u_{1}
\end{array}\right)=\left(\begin{array}{cc}
\cosh (\Upsilon d) & Z \sinh (\Upsilon d) \\
Z^{-1} \sinh (\Upsilon d) & \cosh (\Upsilon d)
\end{array}\right)\left(\begin{array}{l}
F_{2} \\
u_{2}
\end{array}\right)
$$

Where the subscript " 1 " and " 2 " refer to port " 1 " and " 2 " of the layer respectively. 


\subsection{4}

\section{Analytical loss conside rations}

Distinctly from the PSPICE model the analytical model does not use analogies to represent the mechanical part with electrical entities. The piezoelectric losses and the intermediate losses can be more properly formulated in the model. As was done previously, the loss consideration is divided in two parts, piezoelectric and intermediate layer.

\section{a) Piezoelectric transducer}

To model the losses involved in the piezoelectric transducers, it is not common to represent the losses as a single term $\alpha$, real part of the propagation constant $\Upsilon$. Instead, it is adopted the more accurate approach with considers three types of losses. Each of which is associated with three different piezoelectric constants, $\beta_{33}, c_{33}, h_{33}$ [94]. This approach treat loss by introducing lossy terms into these parameters but further setting to zero the original loss term $(\alpha=0)$. These lossy parameters are:

$$
\begin{aligned}
& \widetilde{\beta_{33}^{S}} \approx \beta_{33}^{s}(1+j \tan \delta) \\
& \widetilde{C_{33}} \approx c_{33}^{D}(1+j \tan \phi) \\
& \widetilde{h_{33}} \approx h_{33}(1+j \tan \theta)
\end{aligned}
$$

where the tilde over the constant stand for a lossy constant, $\tan \delta$ is the dielectric loss tangent of the piezoelectric material, $\tan \varphi$ is the elastic loss and $\tan \theta$ the piezoelectric loss. It is also possible to represent the losses with different constants set, namely $\varepsilon_{33}, \mathrm{~S}_{33}$ and $\mathrm{d}_{33}$.

$$
\widetilde{T} \approx \epsilon_{33}^{T}\left(1-j \tan \delta^{\prime}\right)
$$




$$
\begin{gathered}
\widetilde{S_{33}^{\widetilde{E}}} \approx S_{33}^{E}\left(1-j \tan \phi^{\prime}\right) \\
\widetilde{d_{33}} \approx d_{33}\left(1-j \tan \theta^{\prime}\right)
\end{gathered}
$$

where $\varepsilon_{33}{ }^{\mathrm{T}}$ is the material's permittivity under a constant stress, $\mathrm{S}_{33}{ }^{\mathrm{E}}$ is thematerial's elastic compliance under a constant electric field, and $d_{33}$ is the material's piezoelectric charge constant. The two set of lossy tangents are related by the fllowing relationship

$$
\left(\begin{array}{c}
\tan \delta \\
\tan \phi \\
\tan \theta
\end{array}|=| \begin{array}{ccc}
\frac{1}{1-k_{t}^{2}} & \frac{k_{t}^{2}}{1-k_{t}^{2}} & -2 \frac{k_{t}^{2}}{1-k_{t}^{2}} \\
\frac{1}{1-k_{t}^{2}} & \frac{k_{t}^{2}}{1-k_{t}^{2}} & -2 \frac{k_{t}^{2}}{1-k_{t}^{2}} \\
\frac{1}{1-k_{t}^{2}} & \frac{1}{1-k_{t}^{2}} & -\frac{1+k_{t}^{2}}{1-k_{t}^{2}}
\end{array}\left|\begin{array}{l}
\tan \delta^{\prime} \\
\tan \phi^{\prime} \\
\tan \theta^{\prime}
\end{array}\right|\right.
$$

Where $\mathrm{k}_{\mathrm{t}}$ represents the electromechanical coupling factor, thickness mode, of the piezoelectric transducer without loss, which is defined as

$$
k_{t}=\frac{d_{33}}{\sqrt{\left(s_{33}^{E} \epsilon_{33}^{T}\right)}}=\frac{h_{33}}{\sqrt{\left(c_{33}^{E} \beta_{33}^{T}\right)}}
$$

Usually, the $\tan \delta^{\prime}$ and $\tan \theta^{\prime}$ losses did not appear in material properties. However, the dielectric loss tangent $(\tan \delta)$ is common in material datasheets of piezoelectric transducers. The elastic loss tangent ( $\tan \phi$ ') is the inverse of the mechanical usual quality factor $(\mathrm{Q})$.

$$
\tan \phi^{\prime}=Q^{-1}
$$

In this work the following set of parameters $\left(\beta_{33}\right),\left(c_{33}\right)$ and $\left(h_{33}\right)$ were adopted. Therefore, it is convenient to express $\tan \phi$ in function of $\tan \phi$ ', resulting in

$$
\tan \phi=\frac{1-k^{2}}{k^{2}} \tan Q^{-1}-\frac{1}{k^{2}} \tan \delta+2 \tan \theta
$$

The implementation of this losses impacts directly on the A, B, C and D entries of the ABCD matrix, associated in Eq. (10), (15), (21) and (59). To demonstrate it firstly we replace equation (75) in the expression of the speed of 
sound of the piezoelectric transducer and apply the first order Taylor series expansion

$$
\widetilde{U}_{p}=\sqrt{\frac{\widetilde{c}_{33}^{D}}{\rho}} \approx \sqrt{\frac{C_{33}^{D}}{\rho}}\left(1+j \frac{1}{2} \tan \phi\right) \approx U_{p}\left(1+j \frac{1}{2} \tan \phi\right)
$$

Considering the change in the speed of sound, then $\beta$ which is defined in (21) receives a complex term and is written as

$$
\widetilde{\beta}=\frac{\omega}{\widetilde{U}_{p}} \approx \frac{\omega}{U_{p}}\left(1-j \frac{1}{2} \tan \phi\right) \approx \beta\left(1-j \frac{1}{2} \tan \phi\right)
$$

The propagation constant was modeled with the attenuation coefficient equals to zero. However, implying the separated losses in the model transforms $Y$ as fllows

$$
\widetilde{\Upsilon}=j \widetilde{\beta} \approx\left(1-j \frac{1}{2} \tan \phi\right) \approx \frac{1}{2} \tan \phi+j \beta
$$

The effect of the losses in the constant $Z$, defined in Eq. (59), is obtained by using the new expression of $U_{p}$. Thus, the constant $\mathrm{Z}$ can be rewritten as

$$
\widetilde{Z} \approx A \rho \widetilde{U}_{p}(1) \approx A \rho U_{p}\left(1+j \frac{1}{2} \tan \phi\right)
$$

Finally, to determine the impact of the losses in the constant $C_{0}$, defined in Eq. (10), one has to express the permittivity $\varepsilon_{33}$ in function of $\beta_{33}$ as

$$
\widetilde{C}_{0} \approx \frac{\tilde{\epsilon}_{33} A}{d} \approx \frac{1}{\beta_{33}} \frac{A}{d} \approx C_{0}(1-j \tan \delta)
$$

Including these losses in the model affect on four constants presented in the ABCD parameters, namely $h_{33}, \Upsilon, Z$ and $C_{0}$ which are then transformed into new constants that assume complex values. The piezoelectric loss, $\tan \theta$, is normally not provided in the material datasheet. This means that when implementing this specific loss some kind of empirical seek for the better value should be done in order to obtain the proper accuracy of the model, as suggested Lawry[63].

b) Intermediate layer losses

Loss in the intermediate layer is considered by extending the attenuation term of $\Upsilon$. Since it is defined as the sum of viscoelastic attenuation constant $\alpha$ with the 
solution wave number, multiplied by the imaginary unity, $\mathrm{j} \beta$, the diffraction loss $\alpha_{\mathrm{s}}$ is incorporated in $\Upsilon$ by adding to the formers, becoming

$$
\Upsilon=\alpha+\alpha_{s}+j \beta
$$

The diffaction loss attenuation is given by

$$
\alpha_{s}=\frac{-\ln \left(l_{s}\right)}{d}
$$

where $1_{\mathrm{s}}$ is the diffaction loss factor. Following Lawry [63] $1_{\mathrm{s}}$ can be obtained by:

$$
l_{s} \approx \frac{1-\left(\frac{2 \beta^{2} r^{2}-\xi^{2}}{2 \beta^{2} r^{2}}\left[J_{0}(\xi)+i J_{1}(\xi)\right]-\frac{\xi^{2}}{\beta^{2} r^{2}}\left[i \frac{J_{1}(\xi)}{\xi}\right]\right) e^{-i \xi}}{1-\frac{J_{1}(2 \beta r)}{\beta r}}
$$

where $J_{0}$ and $J_{1}$ are Bessel functions of the first kind and $\xi$ is the dummy variable defined as:

$$
\xi=\frac{\beta}{2}\left(\sqrt{\left(d^{2}+4 r^{2}\right)}-d\right)
$$

$r$ is the radius of the transducer.

Similar approach has been used in Pspice model to calculate the diffaction loss, however, there a much simpler equation has been adopted related to the ratio of energy received with energy transmitted on the transducers recall Eq. 41.

The diffraction loss of the analytical model is frequency dependent, which is not possible to be implemented in Pspice due to a limitation in the model.

\subsection{5}

\section{Analysis of power transfer}

As explained in section 3.1.5, for the Pspice mode, evaluations of Sparameters is required to analyze the power transfer of the PDAC system. The main element of the analytical model is the global system ABCD matrix. This is obtained by the multiplication of the individual sub-system ABCD matrices of each layer. The global matrix, which is indeed an electrical-electrical matrix as it begins and ends with the electrical port of both, emitter and receiver, transducers, can be used in order to obtain the S-parameters matrix. Freckey [95] showed that the parameters of $\mathrm{ABCD}$ matrix can be converted to S-parameters as fllows, 


$$
\begin{aligned}
& S_{11}=\frac{A Z_{i}+B-C Z_{o} Z_{i}-D \dot{Z}_{o}}{A Z_{i}+B+C Z_{o} Z_{i}+D Z_{o}} \\
& S_{12}=\frac{2(A D-B C) \sqrt{R_{o} R_{i}}}{A Z_{i}+B+C Z_{o} Z_{i}+D Z_{o}} \\
& S_{21}=\frac{2 \sqrt{R_{i} R_{o}}}{A Z_{i}+B+C Z_{o} Z_{i}+D Z_{o}} \\
& S_{22}=\frac{-A Z_{i}+B-C \dot{Z}_{i} Z_{o}+D Z_{o}}{A Z_{i}+B+C Z_{o} Z_{i}+D Z_{o}}
\end{aligned}
$$

Where $Z_{i}$ and $Z_{o}$ represent the complex electrical impedances of inside and

outside ports, respectively and $\mathrm{R}_{\mathrm{i}}$ and $\mathrm{R}_{\mathrm{o}}$ represent the real part of $\mathrm{Z}_{\mathrm{i}}$ and $\mathrm{Z}_{\mathrm{o}}$, respectively.

The $\mathrm{S}$ parameters have the advantage to be defined in terms of incident and reffected power, as commented in section 3.1.5. Using (93)-(96) it is then possible to evaluate the power transfer of the whole channel modeled with the analytical model.

\subsection{6}

\section{Analysis of data communication}

The data communication analysis has the same purpose of section 3.1.6. For the ABCD matrix approach, the input voltage can be obtained by connecting the global ABCD matrix in series with a $50 \Omega$, which represents the output load from the voltage source, as illustrated in Fig. 3.11. The electrical-electrical 2-port ABCD configuration allows the cascade connection of electric element matrices into the ABCD channel model. The impedance $\mathrm{Z} 0$ represents the load which is connected to the output port, this last can be then switched between distinct values in order to observe the variation of voltage in the input port of the global $\mathrm{ABCD}$ matrix. 


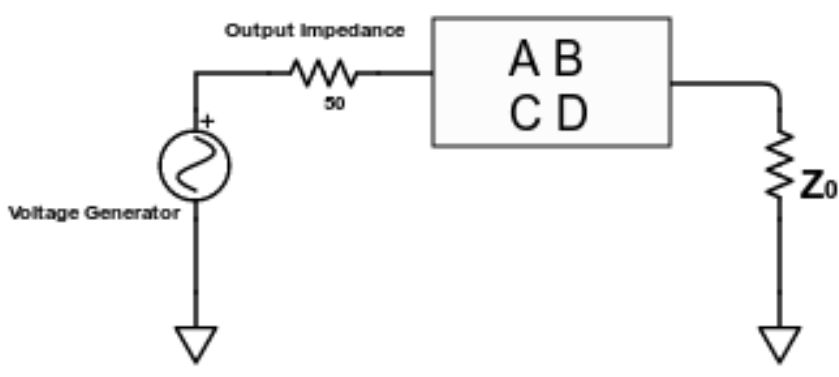

Figure 3.11 Equivalent impedance diagram

Considering that the subscripts 1 stand for the voltage at the source, the subscript $i$ to the voltage at the input port of $A B C D$ matrix, and the subscript 2 to the output port of it, the the electrical-electrical equivalent matrix becomes

$$
\left(\begin{array}{c}
V_{1} \\
I_{1}
\end{array}\right)=\left(\begin{array}{cc}
1 & 50 \\
0 & 1
\end{array}\right)\left(\begin{array}{ll}
A & B \\
C & D
\end{array}\right)\left(\begin{array}{l}
V_{2} \\
I_{2}
\end{array}\right)
$$

where the first matrix in the second term represent the electric matrix of the series connection of a $50 \Omega$ resistor the input port of the ABCD matrix, $V_{1}$ and $I_{1}$ are the voltage and current amplitudes of the voltage source, respectively and $\mathrm{V}_{2}$ and $I_{2}$ are the voltage and current amplitudes at the impedance $Z_{0}$. These are related by

$$
\frac{V_{2}}{I_{2}}=Z_{0}
$$

The input and output ports of the ABCD matrix are related by.

$$
\left(\begin{array}{c}
V_{i} \\
I_{i}
\end{array}\right)=\left(\begin{array}{cc}
1 & -50 \\
0 & 1
\end{array}\right)\left(\begin{array}{l}
V_{1} \\
I_{1}
\end{array}\right)=\left(\begin{array}{cc}
A & B \\
C & D
\end{array}\right)\left(\begin{array}{c}
V_{2} \\
I_{2}
\end{array}\right)
$$

One can observe that the input voltage $V_{i}$ and current $I_{i}$ are related to V1 and I2 by the first equality in Eq. (99). Substituting $\mathrm{V}_{1}$ by $1 \mathrm{~V}$ and applying the relationship of Eq. (98) leads to an expression that depends on the channel ABCD matrix entries and the impedance $\mathrm{Z}_{0}$.

$$
V_{i}=1-\frac{50 Z_{0}[C+D]}{50 C Z_{0}+50 D+A Z_{0}+B}
$$


The input voltage, $V_{i}$ changes when the impedance $Z_{0}$ varies between two values. It is worth mentioning that the analytical model solution is based on frequency domain, and despite being possible to convert to time domain by performing the inverse Fourier Transform, the result would be related with the steady-state of the input voltage when varying the $\mathrm{Z}_{0}$ in two values. In other words, it is not possible to evaluate the transient behavior. Nevertheless, the variation in the frequency domain is carried out.

\section{3 Models verification and comparison}

This section evaluates both models in order to analyze their behavior when varying some parameters and compare them as means of quantifying the capacity of the system. The material properties of piezoelectric transducer, metal layer and adhesive layer from table 3.4, in section 3.1.4 are used in the analytical and numerical models. Thereby, power transfer is evaluated through the S21 parameter fllowing sections 3.1.5 and 3.2.5 for Pspice and analytical model, respectively. Data communication capability is simulated in Pspice and analytically, evaluating the input voltage in frequency and time domain following the sections 3.1.6 and 3.2 .6 , respectively.

The first simulation concerns an acoustic channel composed by Aluminum and adhesive layers. Different thickness of the Aluminum layer were analyzed to observe how the power pass through the acoustic channel, the adhesive thickness is fixed at 100um. Fig. 3.12 shows the S21 values obtained with the Pspice and analytical models, in which continuous line stand so analytical models and dotted lines for Pspice model. 


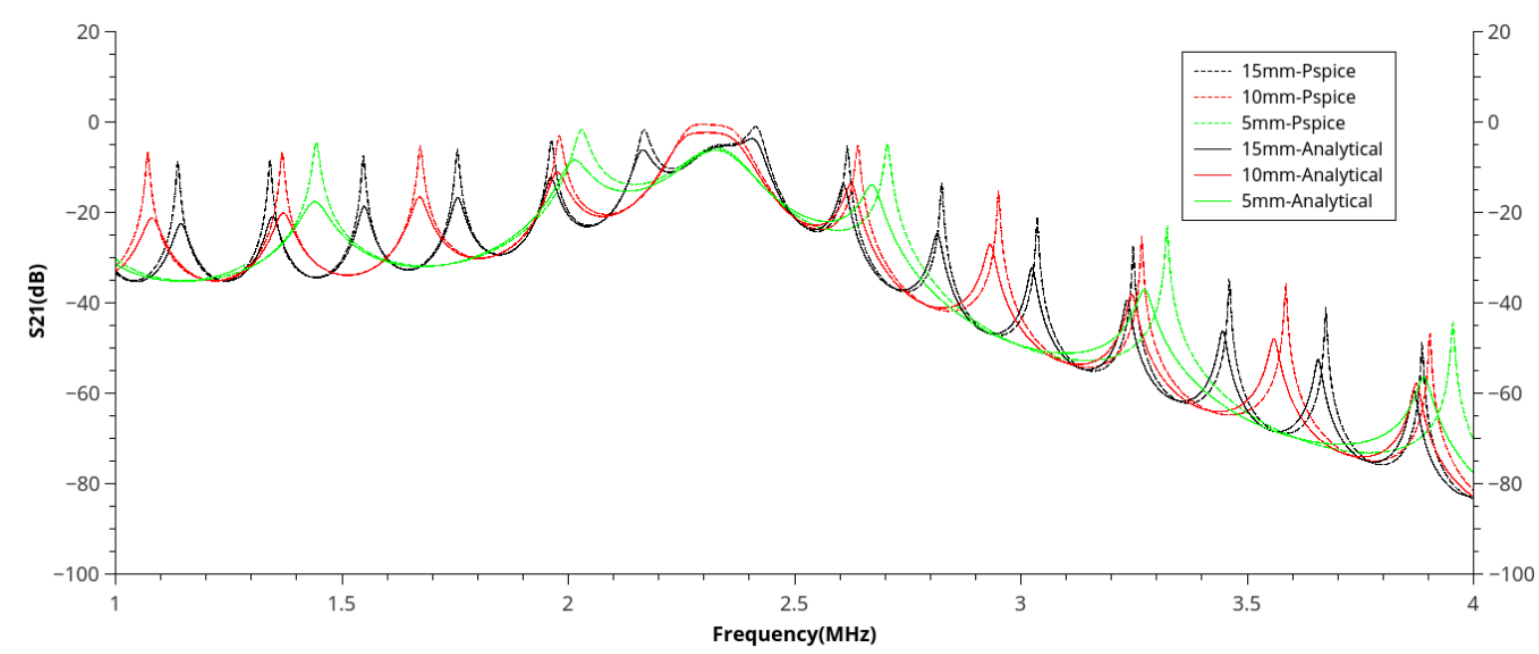

Figure 3.12 Power transfer analysis of both models varying the metal thickness

Fig 3.12 shows a characteristic pattern of an acoustic channel, simulated by both models. The curves show multiple points of resonance and anti-resonance that are related with a multipath propagation. Multipath propagation makes the signal that reach the inside transducer dependent on the phase speed and thickness each layer. One can also notice that increasing the thickness decreases the spacing between the peaks. Since the goal is to transfer the highest quantity of power, the most relevant parameter to evaluate is the frequency which has the highest peak. As demonstrated in the Fig. 3.12 at about $2.27 \mathrm{MHz}$, which is the transducer fundamental resonance, a $5 \mathrm{~mm}$ metal thick has an insertion loss, defined as the loss of power when the wave travels a device, higher than of $10 \mathrm{~mm}$ and $15 \mathrm{~mm}$ thick. It is naturally expected that the longer the metal later is the higher the insertion loss would be since losses addressed in section 3.1.3 and 3.2.4 are dependent on the layer thickness. This is however not observed. The reason for that is that due to the distinct peaks distribution along the frequency spectrum. In the case of $5 \mathrm{~mm}$ thickness an anti-resonance valley coincides with the transducer fundamental resonance frequency, considerably reducing the S21 at this frequency. Similar behavior can happen for different material, due to the difference on the phase speed. This behavior suggests that attention should be drawn since the peak of power transfer may not present a monotonic relationship with the thickness of the layer. The model are thus very useful to design a channel.

The highest value of power efficiency occurs for a $10 \mathrm{~mm}$ thick channel, it has $0.56 \mathrm{~dB}$ of insertion loss at $2.27 \mathrm{MHz}$ in Pspice and $2.33 \mathrm{~dB}$ at $2.30 \mathrm{MHz}$ in the 
analytical model. The maximum power transfer is slightly out of the PZT fundamental resonance frequency mainly because of the attachment of the transducer on the metal, and the resonances distribution in frequency.

A second simulation with a fixed aluminum thickness of $13 \mathrm{~mm}$ and distinct values of adhesive thickness is made, in order to evaluate the impact on power transfer distribution when varying the adhesive thickness. Fig. 3.13 shows Pspice and analytical results for this simulation.

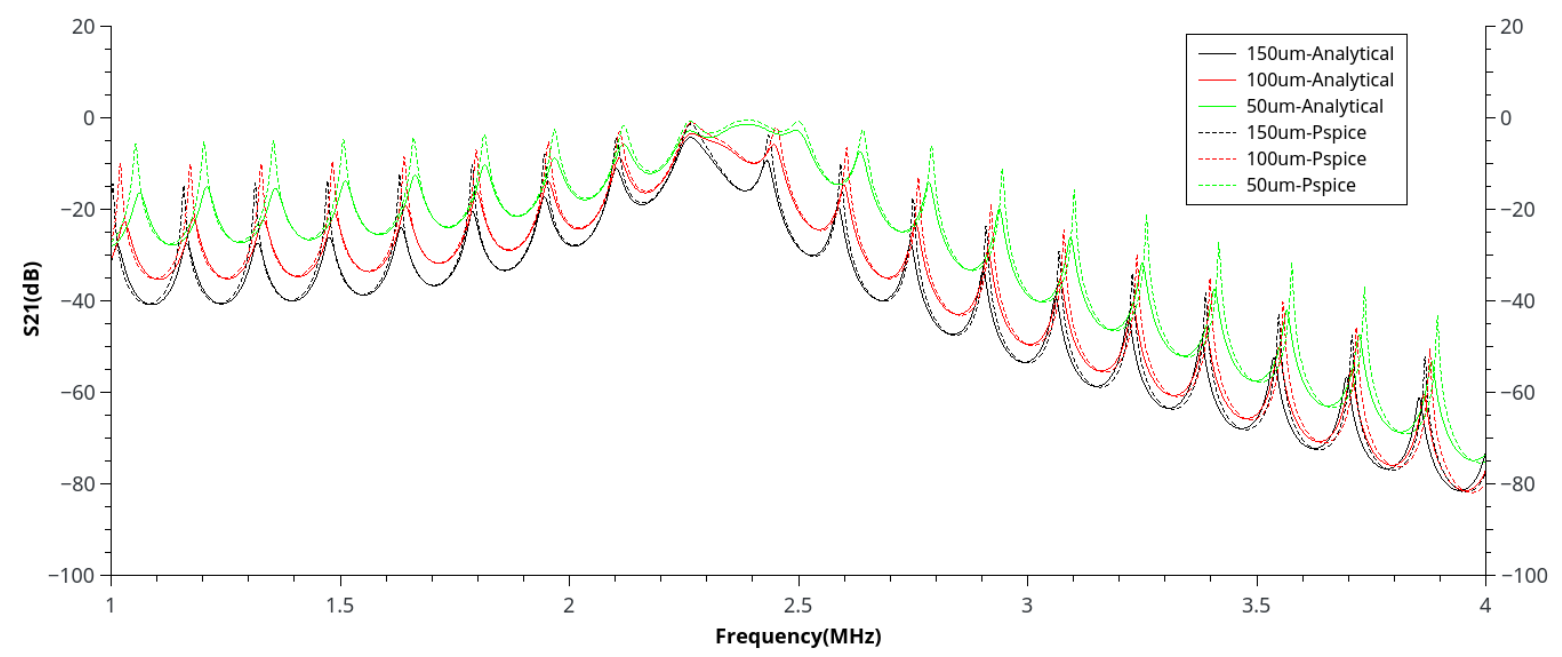

Figure 3.13 Power transfer analysis varying the adhesive thickness

The frequency distribution reveals a dependence of the power with the adhesive as the thickness increases. Distinct from the previously analysis, the S21 shape along the spectrum of frequency is more well-behaved, showing increasing attenuation for increasing adhesive thickness from 50um to $150 \mathrm{um}$ both for Pspice and analytical models. Therefore one can suggest that the adhesive thickness has to be as lower as possible to acquire a good power transfer.

A third simulation is performed to evaluate the communication between the PZT's transducers. The simulation is performed by setting a $13 \mathrm{~mm}$ of metal thickness and 100um of adhesive thickness. Selecting the best point to operate means to have the biggest difference in voltage on the outside transducer when switching the electrodes of the inside transducer between two states or changing the electric resistance in its terminals. In Pspice and analytical, a frequency domain analysis is made, where the voltage on the outside transducer can be monitored switching the inside electrodes between two different resistors $50 \Omega$ and $0.22 \Omega$ Fig. 3.14 show the amplitude on the outside transducer for the load values on the 
inside transducer. Fig. 3.15 shows the difference of amplitude when the electrodes of inside transducer is connected to two different values of resistor.

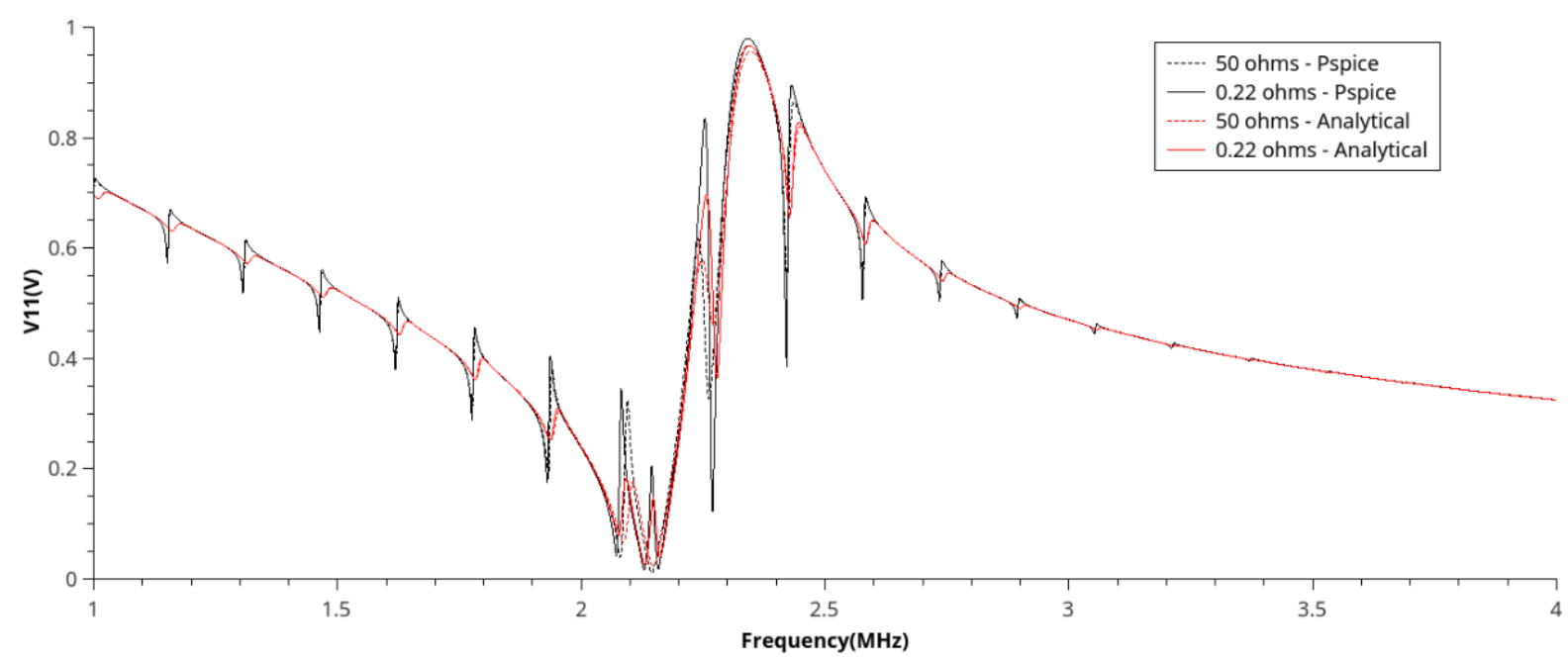

Figure 3.14 Input voltage V11 Pspice and analytical

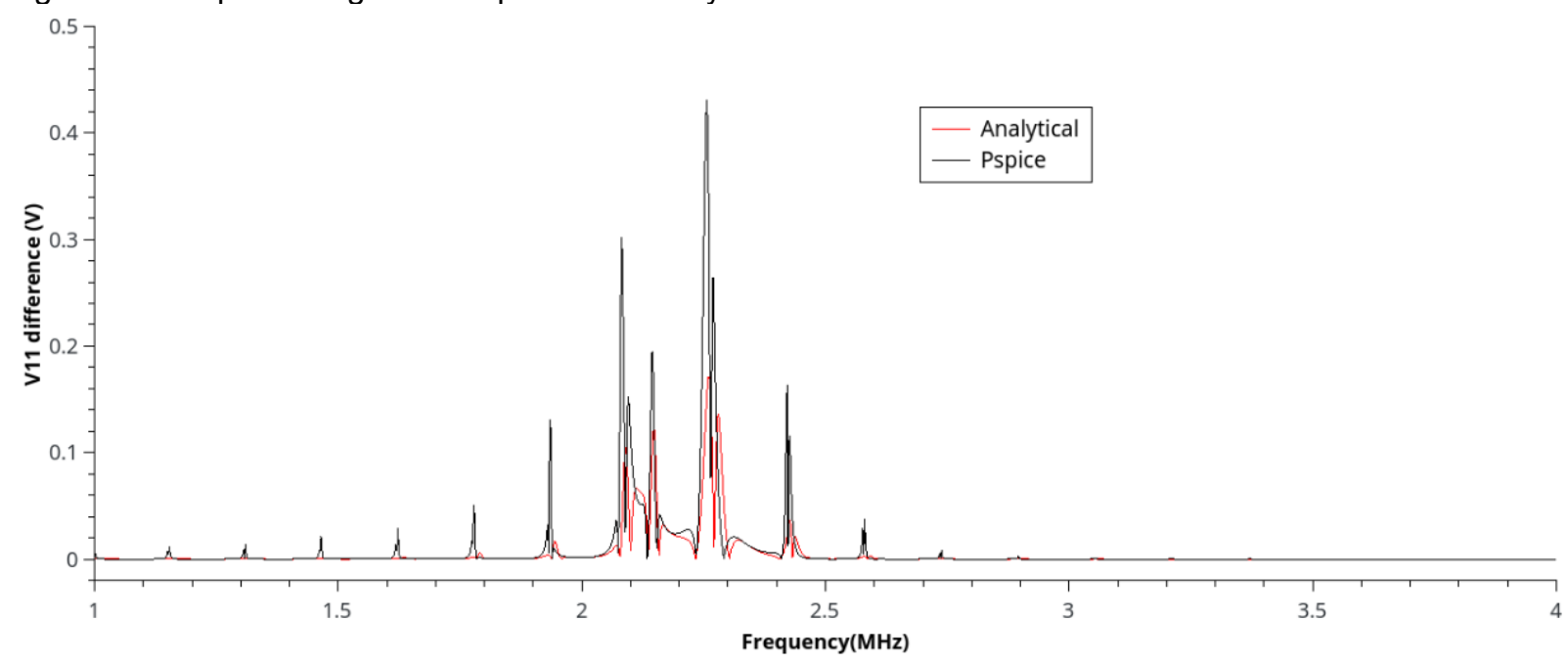

Figure $3.15 \mathrm{~V} 11$ difference, $50 \Omega$ and $0.22 \Omega$ in Pspice and analytical

As can be seen in Fig. 3.15 the acoustic channel is highly selective also in amplitude voltages. The optimal frequencies of operation is found at $2.256 \mathrm{MHz}$, for the Pspice simulation, in which the V11 difference is about $0.4 \mathrm{~V}$. However at the nearby frequency of $2.235 \mathrm{MHz}$ the acoustic channel gives a variation of approximately $0 \mathrm{~V}$. 
The frequency of $2.256 \mathrm{MHz}$ used in the Pspice transient analysis is showed in Fig. 3.16. This operating frequency is the optimal value of this system and was taken from the highest peak of Fig. 3.15. By switching S1 and S2 one can si mul ate a binary digital communication in Pspice. The transient analysis was not implemented analytically as previously discussed in section 3.2.6. Figure 3.16 shows the signal in the time domain, the interval fom 0 to $100 \mu$ s represents the period in which the switch S1 is closed and S2 is open, which means that a resistance of $50 \Omega$ is connected at the inside, and the interval fom $100 \mu \mathrm{s}$ to $200 \mu \mathrm{s}$ represents the period in which the switch S1 is open and S2 is closed, means that a resistance of $0.22 \Omega$ is connected at the inside.

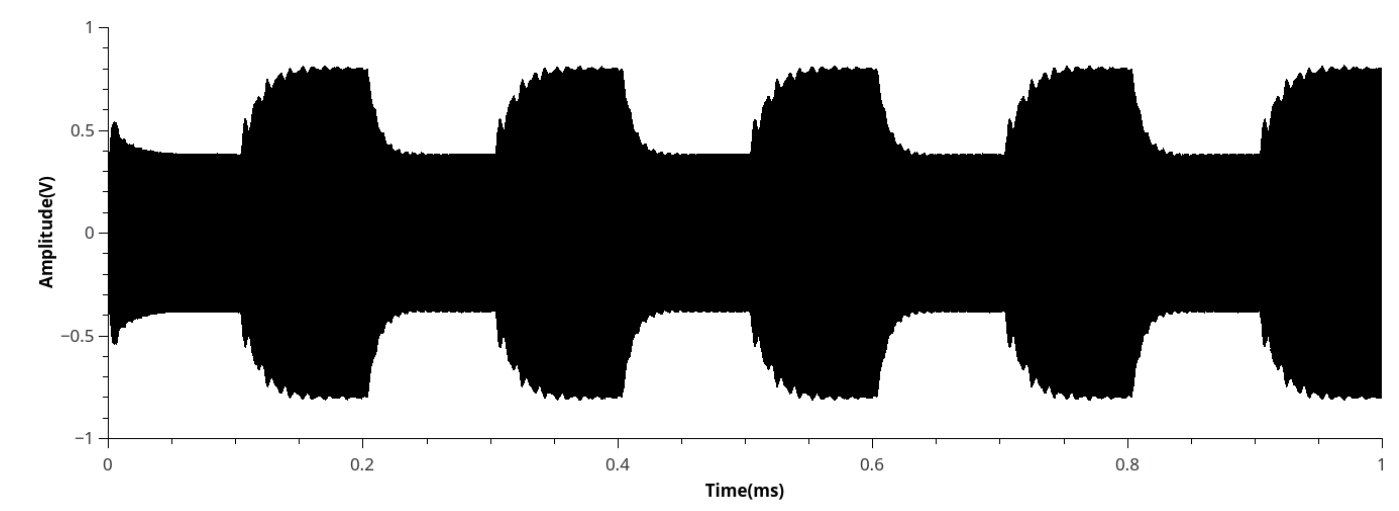

Figure 3.16 Output PZT voltage at $2.256 \mathrm{MHz}$

In order to illustrate the high selectivity of the channel, the same switching scheme is performed at $2.235 \mathrm{MHz}$, which according to Fig. 3.15 shows lower variation. The result is shown in Fig. 3.17. As it can be seem it is almost unfeasible to demodulate this signal

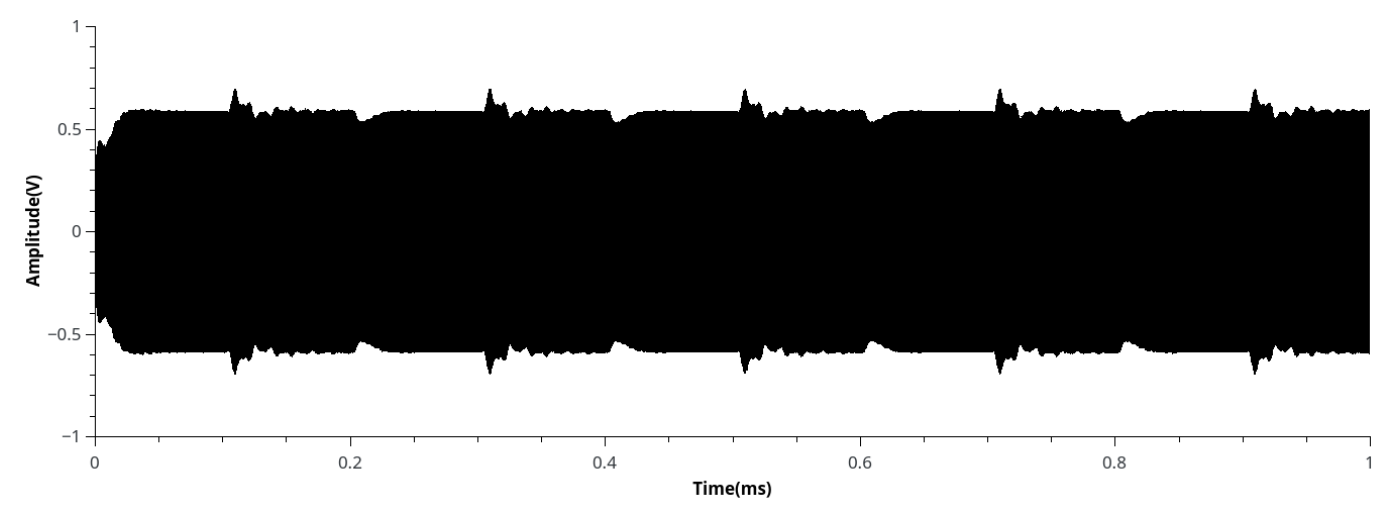

Figure 3.17 Output PZT voltage at $2.235 \mathrm{MHz}$

One can conclude that for both Pspice and the analytical models of PDAC system, 
the shape of S21 parameter, with highly selective peaks and vall eys are nearly coincident. This suggests that both models, although of different approaches, can simulate acoustic channel and piezoelectric behavior of the PDAC. Nevertheless, the values of the peaks and valleys have slightly difference when comparing Pspice and analytical model, this is mainl y because of the different loss implementation in each simulation. The Pspice model has four types of loss, two in piezoelectric transducer and two inintermediate layer but none of them are frequency dependent. The analytical model, on the other hand, has five types of loss, three in piezoelectric transducer and two in intermediate layer, where the diffraction attenuation is fequency dependent.

The transient results reveal the high difference of amplitude voltage in the output PZT when the system is operating with two distinct frequencies. Using a frequency of $2.256 \mathrm{MHz}$ the amplitude varies $0.4 \mathrm{~V}$ but at $2.235 \mathrm{MHz}$ the amplitude has a minor variation, showing that the system is highly selectivity.

In the next chapter evaluation and comparison between both models and between experimental tests are performed, in which models use the same scenario of the experimental setup, comprising metal and adhesive layers. In Pspice a more complete circuit is connected on the acoustic channel, developed in this chapter, in order to simulate the power and data transfer. The full circuit is capable of simulating a digital modulation and demodulation, and powering all the inside block electronics. 


\section{4}

\section{PDAC implementation}

In chapter 3, an equivalent circuit for the acoustic channel with mechanicalto-electrical analogy was developed. Designing the circuit in PSPICE allows one to evaluate the power and data communication transfer and to perform transient analysis. The overall circuit diagram (see Fig 3.1) illustrates the main elements coupled to the acoustic channel block; namely, signal generator, RF amplifier, envelope detector, voltage comparator, Moset transistor switch, energy harvesting circuitry, microcontroller and sensors. These elements, either in the outside or inside block, are necessary to provide power to the whole system and to modulate and demodulate the sensors data. Most of these elements can be approximately simulated in PSPICE by means of similar functions.

The type of modulation adopted in thi s work was Amplitude-shif keying (ASK). In the next sections an introduction to ASK modul ation is presented as well as the developed el ectronics. The full system circuit and all the necessary elements to modul ate and demodulate the sensor data are described. The concepts of the mainly elements of both, outside and inside blocks are also presented.

\section{1}

ASK

ASK belongs to the group of digital modulation. It is composed by a carrier signal, a modulating signal and a modulated signal. The diagram of Fig. 4.1 illustrates these three signals. Distinctly fom analog modulation in which the carrier signal is modulated by a continuous signal, in the digital modulation a discrete signal is used for modulating. Therefore, the carrier can be, for example, modulated straightly from digital sensors data. The modulating signal is multiplied by the carrier signal generating the modulated signal, Generally, in practice the carrier frequency is a sinusoid wave with a frequency higher than the data baud rate to avert the noise interference [101]. 


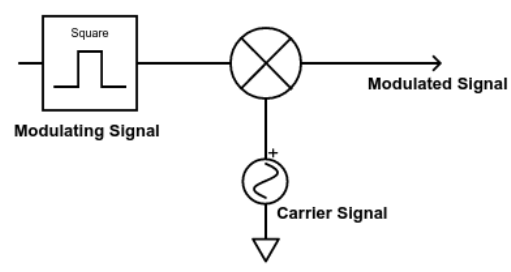

Figure 4.1 ASK modulation diagram

The modulating signal, or the sensor data information, is reconstructed using an envelope detector circuit followed by a voltage comparator when the modulated signals goes through the acoustic channel. One advantage in the use of a carrier instead of simply transmitting the data in base band is that it can be generated in the outside block, where there is no power restriction. The ASK demodulation has the ability to be simple but efficient for low data rates, with few components the modulating signal can be reconstruct in the other side of the channel.

\section{2 \\ Outside block circuit}

The outside block has four main elements, namely signal generator, RF amplifier, envelope detector and voltage comparator. The signal generator is responsible for creating the carrier signal which is introduced into the acoustic channel. The carrier frequency is to be previously selected from the AC analysis, as presented in chapter 3 .

Communication between the sensors, located in the inside block, and the outside block relies on an amplitude modulation scheme, more precisely a digital amplitude modulation (ASK), presented in section 4.1. Envelope detector and voltage comparator are then necessary to implement the ASK demodulation circuitry.

The circuit in Fig. 4.2 il lustrates the envelope detector implementation, which consists of a diode and a resistor-capacitor, $\mathrm{RC}$ low pass filter. The circuit operation is as follows. Firstly, consider the positive halfcycle of the modulated signal. At this stage the diode is forward-biased and the capacitor is charging at the same rate of the semi-cycle, if this is an ideal diode the stored voltage in the capacitor is the peak value of the modulated signal. When the modulated signal decreases to a 
negative level, the diode becomes reversed-biased and the capacitor discharges through the resistor until the next positive cycle. Assuming this behavior, the RC filter must be designed to have a time constant lower than the inverse of the modulated signal bandwidth and greater than the inverse of the carrier signal frequency [101], i.e.,

$$
\frac{1}{f_{c}} \ll R C \ll \frac{1}{W}
$$

where $f_{c}$ and $W$ are the carrier frequency and the bandwidth, respectively. According to Eq. (101), resistor (R) and capacitor (C) of the RC filter, can be chosen to properly demodulate the sensor signal.

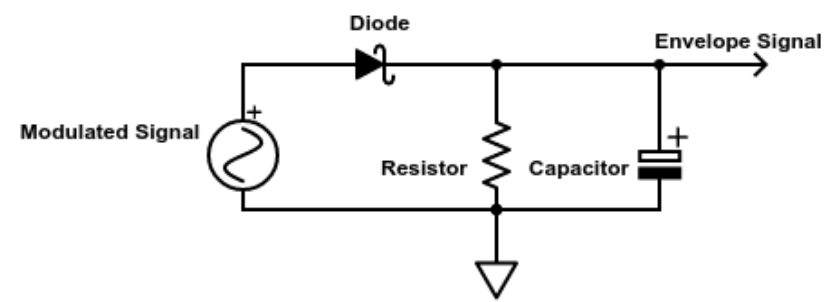

Figure 4.2 Envelope detector schematic

The Fig. 4.3 shows an example of an ASK modulated signal with a carrier frequency operating at $3 \mathrm{Mhz}$ and the envelope signal as a simple digital signal with 150kHz. The black line stands for the envelope signals and the red line to the modulated signal.

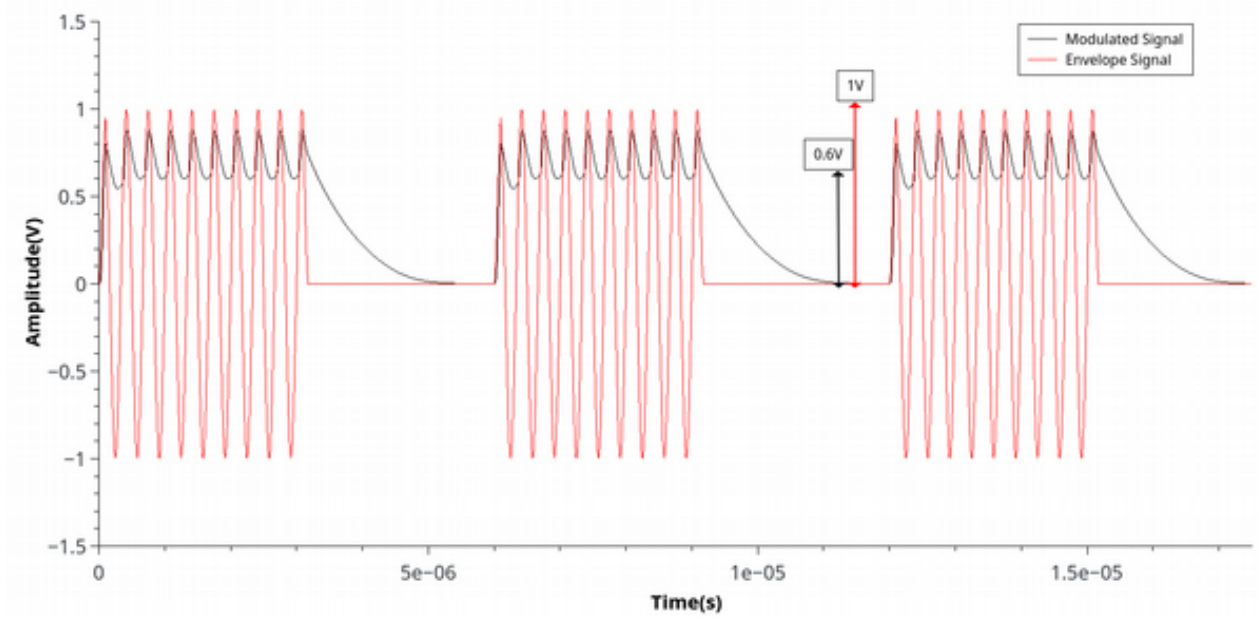

Figure 4.3 Envelope Signal and Modulated signal example 
One can observe that only the positive halfcycle of the modulated signal is demodulated and a lower peak voltage, Vpeak, is reached as the envelope detector has a non-ideal diode. The peak value, which originally is $1 \mathrm{~V}$, affer the diode drops to $0.8 \mathrm{~V}$, approximately. It is also noticeable the charge and discharge of the capacitor on the resistor. This rate is determined by the values of the RC filter. Decreasing the constant time directly impacts the time to reach the high logical level, whereas increasing it affects the low logical level. The chosen RC values for this example gave a high level of 0.6V, black ffag in Fig. 4.3 and a low level of $0 \mathrm{~V}$ with a high to low transient time of 2 us.

As it can be seen, the envelope detector provides the amplitude variation which is related to some information, for instance from a sensors whose output data is used to modulate the carrier at the input port.

Since the acoustic communication of the system is digital, as the sensor data is digitized, the envelope signal is connected to a voltage comparator that reconstructs the digital information of the sensors. A simple voltage comparator can be made using an operational amplifier [102], illustrated in Fig. 4.4. This component works with two inputs one being a refrence voltage and the other the signal to be compared, in this case the envelope signal. When the envelope signal, is greater than the reference voltage, the output of voltage comparator is high $(\mathrm{V}+)$, when the envelope signal voltage is lower than the refrence voltage, the comparator output signal is low (V-).

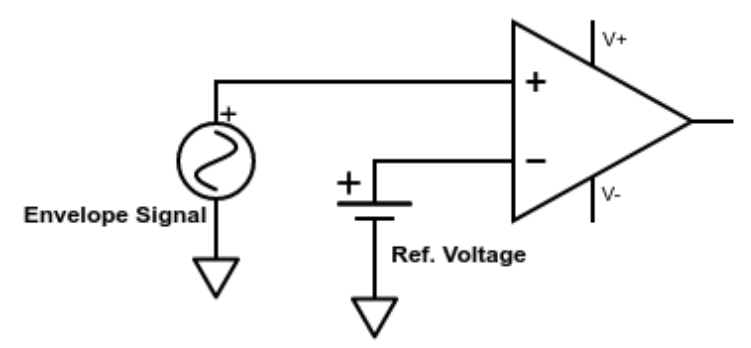

Figure 4.4 Voltage comparator diagram

Passing the envelope signal through the voltage comparator results in a digital signal in the output port. Ideally, the output signal is $5 \mathrm{~V}$ when the original datum is in logical level high and approximately $0 \mathrm{~V}$ when it is in low. These levels depend 
on the supply voltage of the operational amplifier. This functionality turns the voltage comparator into a digital converter.

Fig. 4.5 ill ust rates the envelope signal, the threshold level, the comparator output signal and the modulating signal. In this example the reference voltage is $0.5 \mathrm{~V}$, which is the voltage that the envel ope si gnal changes from logical level.

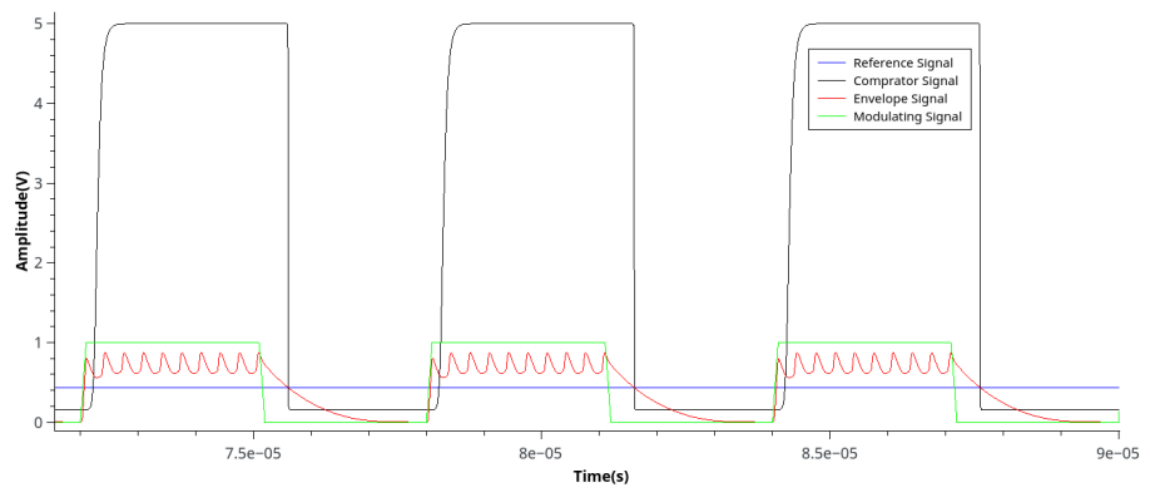

Figure 4.5 input and output signals of the voltage comparator

The comparator signal has 500ns of difference from the modulating signal when the logical level changes from high to low, this fact is caused by the voltage reference chosen and the time response of the voltage comparator. This difference directly impacts the maximum data rate for a given carrier signal.

Therefore, the outside block is not only responsible to supply power to the outside PZT transducer and, consequently, to the inside block, but also to recover the modulated data from the sensors located in the inside block.

\section{3}

\section{Inside block circuit}

The inside block is designed to work passively, i.e. it operates without any conventional power source. The power that supplies the sensors and microcontroller comes from the electrical signal at the inside PZT transducer terminals. This electrical signal comes from the acoustical (mechanical) waves that impinge the transducer face and are then converted to voltage difference between its terminals.

To transform the AC signal received in the terminals of the PZT transducer into a DC source capable of supplying energy to all other elements in the inside block, a voltage doubler circuit is adopted [103]. The topology used is showed in Fig. 4.6. 


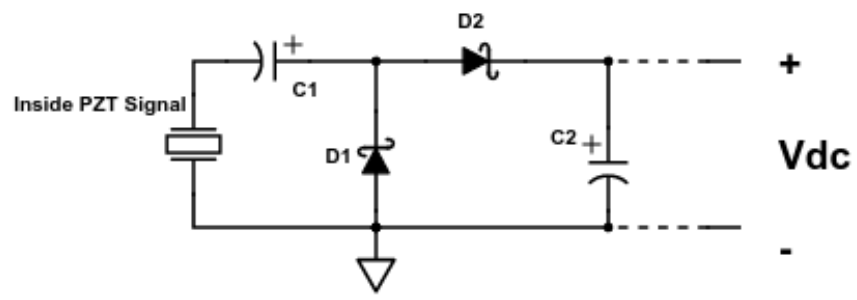

Figure 4.6 Voltage doubler topology

With two schottky diode-capacitor pairs the fundamental operation of the circuit is to multiply and rectify the input AC voltage to give a DC output. In other words, the peak voltage of the RF carrier is multiplied by a factor of two using the topology of Fig. 4.6. Taking into account the voltage drop of both diodes, the output Vdc is,

$$
V d c=2 * V_{\text {peak }}-2 * V f_{\text {diode }}
$$

where $\mathrm{V}_{\text {peak }}$ is the $\mathrm{AC}$ signal on the inside PZT and $\mathrm{Vf}_{\text {diode }}$ is the diode forward drop voltage.

The output Vdc has a voltage that can vary depending on the PDAC environment and for this reason a voltage regulator is introduced to hold the output voltage constant, regardless the value of the obtained Vdc. The components in the inside block (sensors, microcontrollers and other IC's) can have different ranges of input supply voltage and therefore multiples voltage regulator with different output voltage can be used, such as $3.3 \mathrm{~V}$ and $5 \mathrm{~V}$.

The inside block transmits the sensors' data to the outside block by switching the inside PZT terminals between two states, open and short-circuited. To perform this procedure a MOSFET transistor with a low resistance of conduction must be used. That is, when it is switched on, a minimum series resistance between the terminals of PZT is achieved, approaching an ideal short circuit.

In Fig. 4.7, the RF carrier signal obtained at the inside PZT is connected in parallel with the MOSFET. The digital sensor data tums the MOSFET "on" and "of' as the high and low voltage levels reaches the gate port of MOSFET, respectively. 


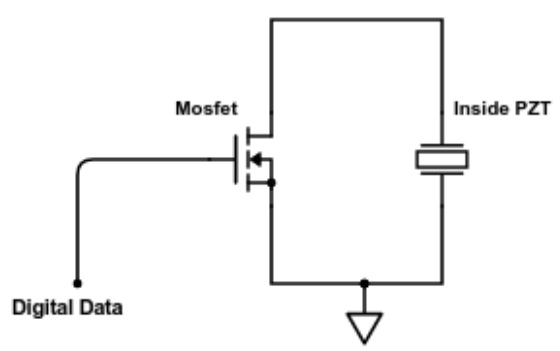

Figure 4.7 Mosfet switching RF carrier

\section{4}

\section{Full system simulations}

It is possible to simulate the PDAC with the peripherals circuits of outside and inside blocks. Using a PSPICE simul ator the circuit is designed as shown in Fig. 4.8. The PDAC system has exactly the same characteristics presented in the chapter 3 and the materials which compose the acoustic channel have the properties of table 3.3 with $10 \mathrm{~mm}$ of aluminum thickness and $100 \mathrm{um}$ of adhesi ve thickness.

In the circuit simulation, the output block, black rectangle in Fig. 4.8, is formed by a simple AC source operating at $2.25 \mathrm{MHz}$, which produces the highest variation in amplitude for the Pspice simulation, see section 3.3. The AC source has $30 \mathrm{Vp}$ and a $50 \Omega$ out put impedance si mul ating the output of a RF ampl ifer.

Additionally to the presented envelope detector in section 4.2, here others components were included to ensure the proper functionality of the ASK demodulation, fr instance, a low pass fil ter and a DC blocker.

In the inside block, red rectangle in Fig. 4.8 the volt age doubler has two schot tky diodes and two capacitors where $\mathrm{C} 1$ and $\mathrm{C} 2$ are chosen to optimize the power transfer. The $5 \mathrm{~V}$ vol tage regulator used is the LT 1117-5 fom Linear Technology ${ }^{\circledR}$ [104]. The MOSFET transistor has an extremely low resistance, $0.22 \Omega$ when conducting, given the sufficient path to ground.

The digital out put part of circuit, light green rectangle in Fig. 4.8 simulates the microcontroller and sensors. Its power consumption is approximatel y $150 \mathrm{~mW}$ when driving the MOSFET and the data rate is $10 \mathrm{kHz}$ in cont inuous mode.

\section{5}

\section{Full system simulation results}

In this section, the results obtained from PSpice simulation of both, modulation and demodulation circuits and the acoustic channel are presented. 


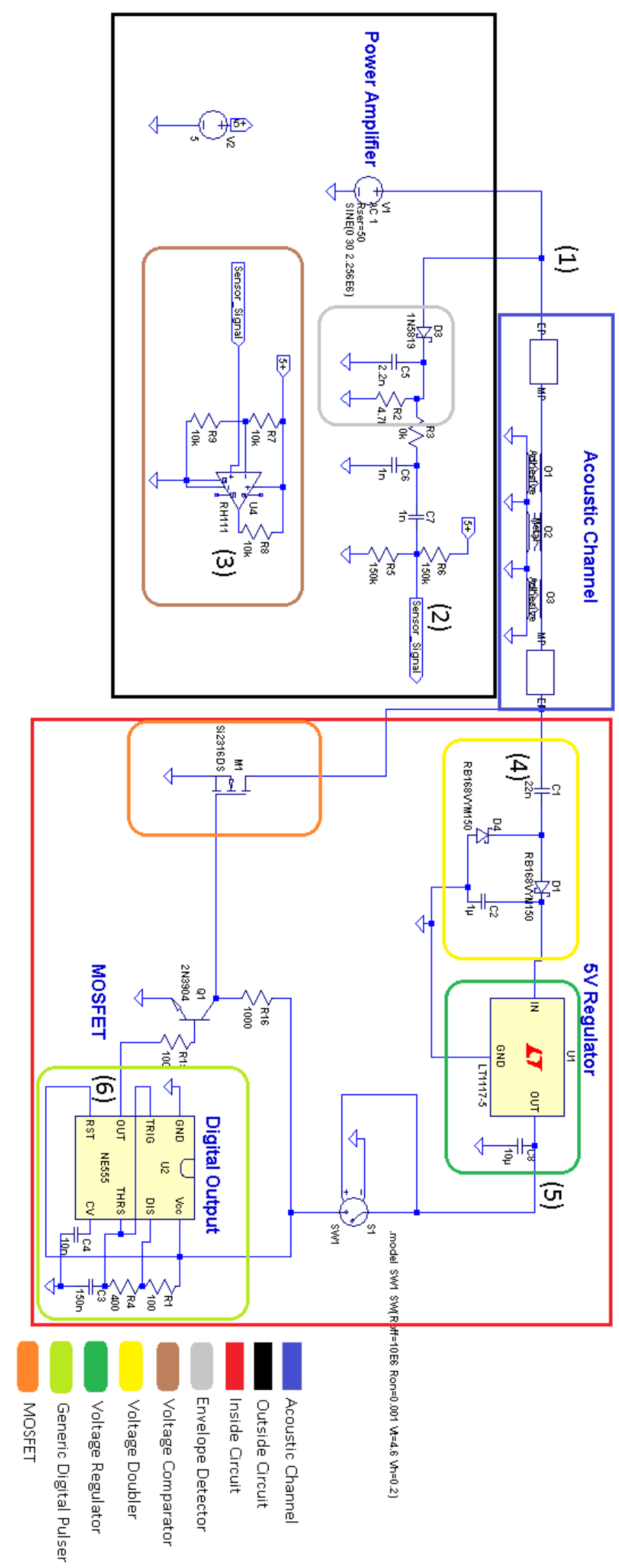

Figure 4.8 PDAC full system with electronic peripherals circuits 
Some critical signals from the circuit are illustrated below to validate the use of the electronic components associated with the outside and inside blocks.

\subsection{1}

\section{Outside block simulation}

The outside block is the black rectangle in Fig. 4.8. One of the most important signals of the outside block is the modulated signal connected between the $50 \Omega$ impedance of the signal generator output and the electrical port of the output PZT; the electric node of this is signal marked with number (1) in Fig. 4.8. This signal is illustrated in Fig. 4.9.

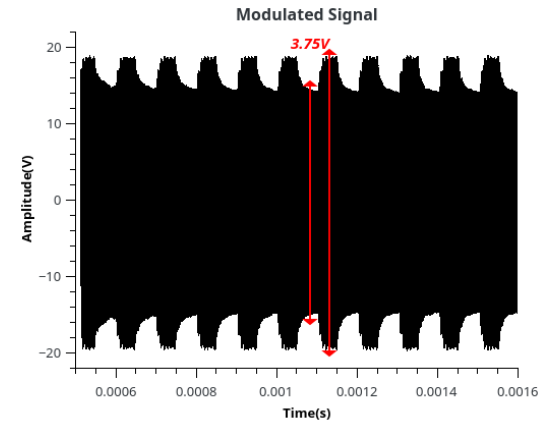

Figure 4.9 Modulated signal of full system

The modulation produced by the digit al output gives a $3.75 \mathrm{~V}$ between high and low voltage levels operating at $2.256 \mathrm{MHz}$. The modulated signal passes through the envelope detect or circuit reconstructing the sensor data information, number (2) in Fig. 4.8. The demodulated si gnal output, bi ased to $2.5 \mathrm{~V}$, can be seen in the Fig. 4.10 .

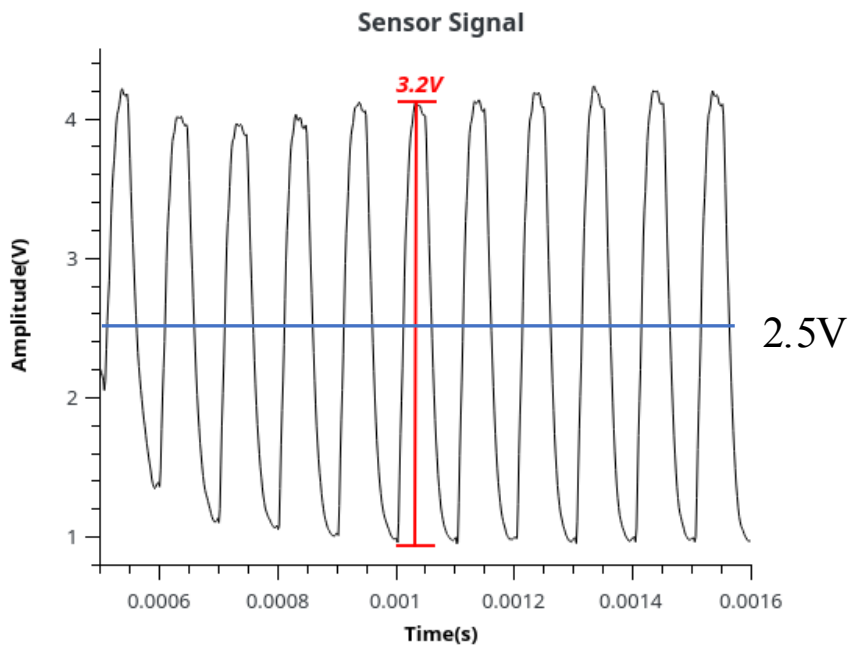

Figure 4.10 Sensor signal of full system 
Comparing Fig. 4.9 and 4.10 one can see that the peak-to-peak voltage of the recovery signal is $0.55 \mathrm{~V}(=3.75 \mathrm{~V}-3.20 \mathrm{~V})$ lower than the difference from the high to low values of the modulated signal. This lower voltage is due to the diode voltage drop and the low pass filter attenuation, necessary elements for the operation of the envelope detector. One can also notice that the first levels are not stabilized, this is due to circuit and acoustic time responses.

Following the circuit, there is the voltage comparator signal, number (3) in Fig. 4.8. As presented in section 4.2, it transforms the sensor signal into a digital signal with two voltage levels, namely $0.2 \mathrm{~V}$ and $5 \mathrm{~V}$. The signals is commuted between these levels at exactly the same data rate of the sensor. This signal is shown in Fig. 4.11,

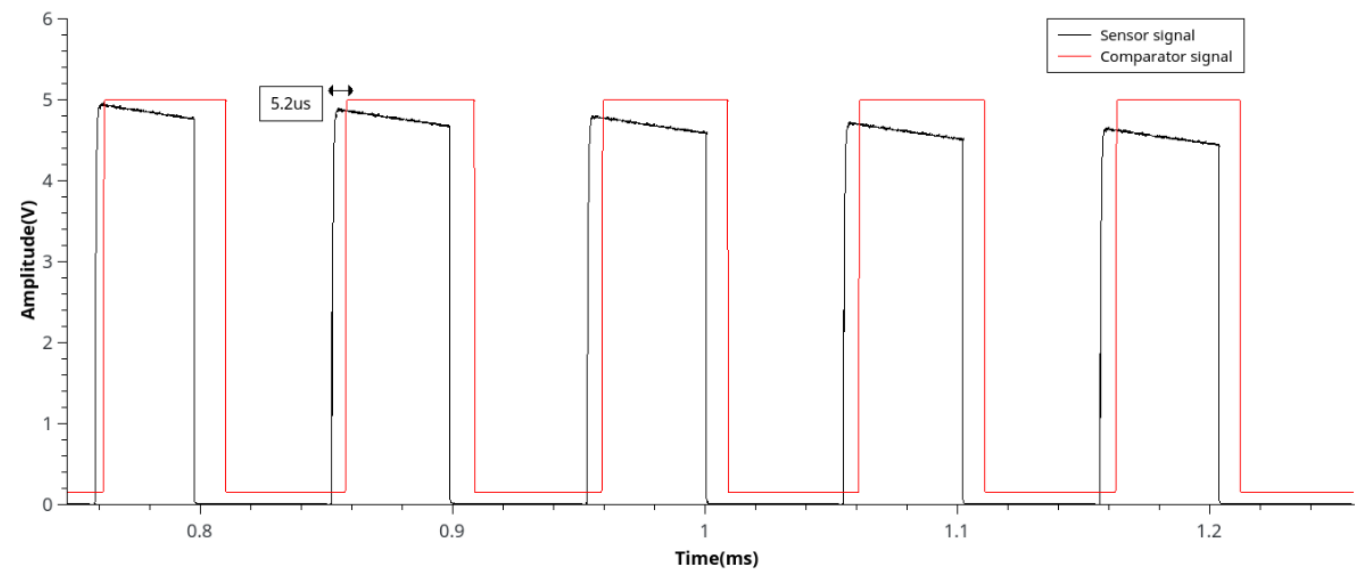

Figure 4.11 Comparision between sensor information and comparator signal

The reference voltage is fixed in $2.5 \mathrm{~V}$ due to the voltage divider composed by the R7 and R9 elements of Fig. 4.7. This level is chosen because it lies at about the middle of the maximum and minimum level of the sensor signal The comparator signal turns the sensor signal of Fig. 4.8 into square pulses with the same data rate, reconstructing the digital information of the sensors.

\subsection{2}

\section{Inside block simulation}

In the inside block some useful signals can be collected to show the functionality of the PDAC and the inside electronic peripherals. In Fig. 4.8, a digital pulser simulates the consumption and data transmission of the sensors and 
microcontroller of PDAC. (this is illustrated by the rectangle in lime color). The digital pulser is only turned on, by the switch SW in Fig. 4.8, when 4. 6V levels is reached in the output port of the voltage regulator, this provides the proper time to the system to stabilize itself before starting the communication. In practice this element is not present in the real circuit, but for the purpose of Pspice simulation it helps the stabil ization and convergence of the simulation.

The Fig. 4.11 shows the sensor information that is transmitted from the inside block to the outside block, number (6) in Fig. 4. 8, in red line. The output of the digital pulser is connected to a BJT transistor that inverts the digital signal and drives the MOSFET, which in turn shorts or opens circuit the inside PZT to ground, respectively. Aiming to compare the signal received in the outside block, the comparator signal is also ill ust rated in Fig.4.11 in black line.

One can notice that when comparing the sensor infrmation with the output of the comparator, the signals are shiffed in time. This delay, approximately of 5.2us, is associated with the wave propagation ti me inside the aluminum, speed of $6420 \mathrm{~m} / \mathrm{s}$ and thickness of $10 \mathrm{~mm}$, accounting $1.6 \mathrm{us}$, the delay of the envelope and low pass filter and the delay of the vol tage comparator. As the waves pass through a physical medium there is a time for them to propagate through the layers (according to the sound speed in each material), causing a shiff in time.

The voltage at the inside PZT electrodes (number (4) in Figure 7) is shown in, Figure 4.12,

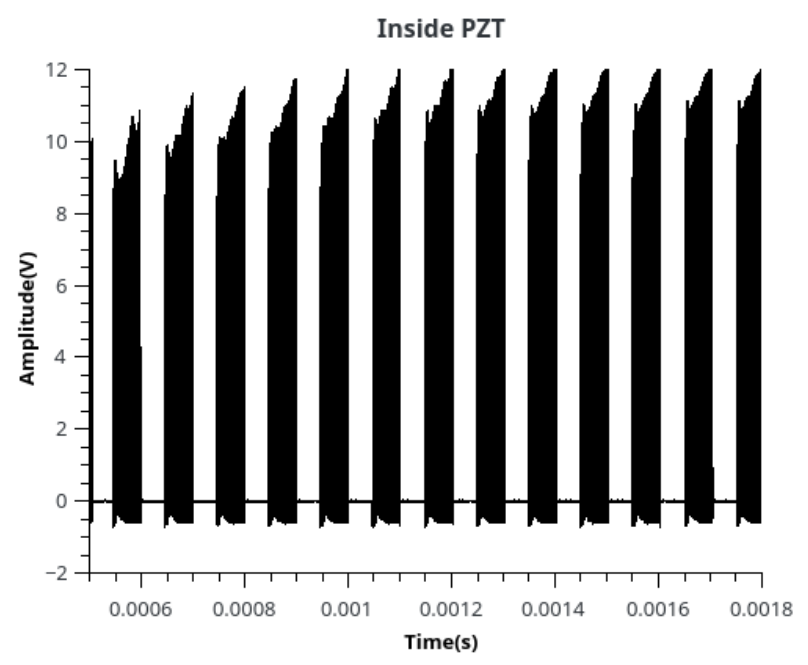

Figure 4.12 Inside PZT signal

The amplitude of the inside PZT gets null when the digital pulser of Fig. 4.9 is in low level, activating the MOSFET. The process is reverted when the digital 
pulser is in high level, deactivating the MOSFET. The inside PZT signal in this stage has a $\mathrm{Vp}$ of approximately $12 \mathrm{~V}$. One can also notice the signal stabilizing after some periods since the system has been switched on. Other characteristics is the suppression of the negative part of the signal when the MOSFET switch is deactivated. This is caused due to the MOSFET intrinsic diode between drain and source when in off state.

As the inside PZT has a volt age drop every time the sensor data is in low state, it is not possible to supply power to the system without the use of a recti fier circuit and a voltage regulator.

Fig. 4.13 shows the behavi or of the output voltage, number (5) in Fig. 9. While the steady state is not achieved the output voltage increases until, some periods have passed and then a $5 \mathrm{~V}$ voltage source, rectified and filtered is established. The absci ssa scale of fgure 4.13 is exactly the same of figure 4.12.

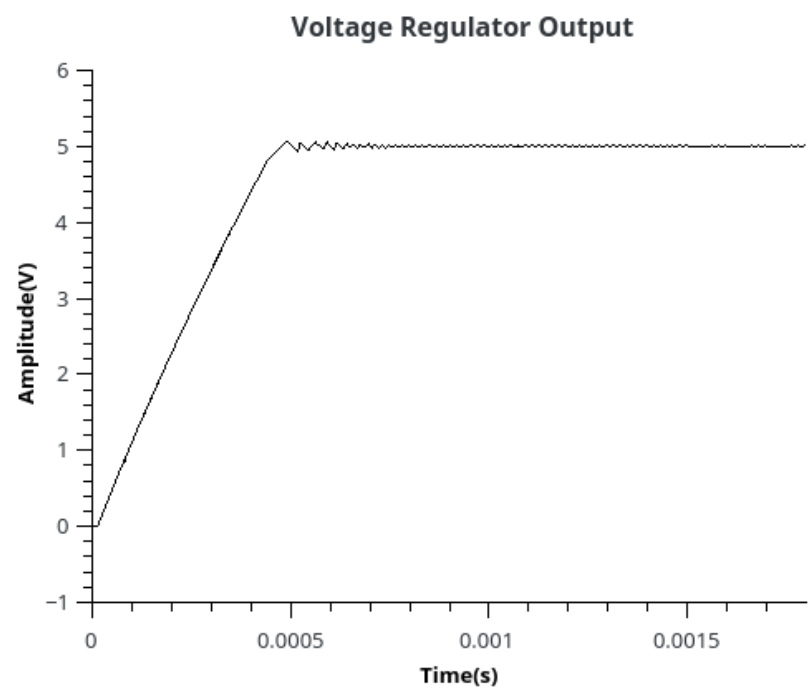

Figure 4.13 5V voltage regulator output

\section{6 \\ Experimental setup}

Up to this point, the dissertation has presented two models, one numerical and the other analytical. The former uses the analogy between electrical and mechanical to create an equivalent electrical circuit that can be simulated using circuit simulation soffware, for instance Pspice. The latter is based on 2-port ABCD matrix with the intermediate layer being modeled by the wave propagation equation and the piezoelectric layer modeled by the constitutive piezoelectric equations. 
Experimentally, the power transfer analysis is assessed using a Vector Network Analyzer (VNA). This equipment is capable of measuring the transmitted signals between two ports and the reffected signal of an individual port. The VNA used in the experimental part is E5061B from Keysight ${ }^{\circledR}$ which operates from $5 \mathrm{~Hz}$ to $3 \mathrm{GHz}$. The parameters that quantify the transmited (S21) or reffected (S11) power used by the VNA were previously discussed in the section 3.1.5. The Sparameters give the necessary information to evaluate the power transfer of experimental system.

The data communication analysis also uses the VNA, however an impedance analysis is performed to extract the voltage difference in the output PZT when switching the inside PZT. Thus, it is possible to evaluate the operational frequency that has the major difference in amplitude and consequently a better data communication point. Affer that, an analysis using the oscilloscope and a signal generator allows the evaluation of a transient characteristics of the PDAC, in this configuration, i.e. a fixed frequency is set and the inside PZT switched by a Moset transistor.

This section is divided in two experiments. The first consists of a flat plate sandwiched by two PZT transducer. The other by a layer of water and a curved surface made of metal (which actual ly is a pipe wall) in the middle of two PZT transducers.

\subsection{1 Flat plate}

This section describes the experimental approach designed to validate the analytical and numerical analysis presented in chapter 3. The first experiment consists of a flat plate made of Aluminum with $13 \mathrm{~mm}$ and a pair of PZT4 transducers from PICeramic $\mathbb{R}[105]$ operating at $2 \mathrm{MHz}$. The PZT transducers and the Aluminum plate were bonded together with an Araldite epoxy adhesive from Hunstaman ${ }^{\circledR}[106]$ in such way that the transducers are co-aligned. Transducer bonding position was determined by previously measuring, with a ruler, and marking the desired position for the center of each transducer on the plate's surfaces. 
In this experimental setup the thickness of the adhesive layer, after the cure process, was measured in 150um, using a mi crometer. The PZT4 transducer has a nominal resonance fequency of $2 \mathrm{MHz}$ given by the manufacturer and a thickness of $1.00 \mathrm{~mm}$, measured using a micrometer. The Aluminium thickness is $13.05 \mathrm{~mm}$, also measured using a micrometer. The properti es of the PZT can be found in the PI Cerami c material datasheet however [113], a charact erization is made using the VNA, a micrometer and a scale.

The method of characterization follows the following steps:

- Measurement of the resonance and anti-resonance frequencies,

- Measurement of the capacitance in $1 \mathrm{kHz}^{3}$,

- Measurement of the impedance value at the resonance peak,

- Measurement of the thickness and area of PZT transducers.

Affer this procedure a list of parameters is calculated as mechanical quality (Qm), elastic stiffness $\left(\mathrm{C}_{33}^{\mathrm{D}}\right)$, thickness electromechanical coupling constant $\left(\mathrm{k}_{\mathrm{t}}\right)$, wave velocity $(v)$, permittivity $\left(\varepsilon_{33}\right)$ and piezoelectric pressure constant $\left(h_{33}\right)$. The density $(\rho)$ was measured using the micrometer and a scale. The equations to calculate these parameters are located in [107].

The Aluminum plate and the adhesive were characterized using a pulse-echo method to acquire the speed of sound and the viscous attenuation. The data of the pulses were measured using an oscilloscope (Tektronix ${ }^{\circledR}$ DPO4054) and the pulse was generated and pre-amplified with The Pulse-Receiver (Olympus ${ }^{\circledR}$ 5072PR). The table 4.1 summarizes the parameters experimentally acquired. All the parameters were measured unless otherwise stated.

3 Since the PZT is mostly capacitive in low frequency, the capacitance determination is measured in $1 \mathrm{kHz}$. 
Table 4.1 Material properties

\begin{tabular}{|c|c|}
\hline \multicolumn{2}{|c|}{ Piezoelectric } \\
\hline Property (units) & $\begin{array}{c}\text { PZT4 } \\
\text { (PiCeramics) }\end{array}$ \\
\hline$\rho\left(\mathrm{kg} / \mathrm{m}^{3}\right)$ & 7641 \\
\hline$v(\mathrm{~m} / \mathrm{s})$ & 4525 \\
\hline Qm & 1500 \\
\hline$\varepsilon_{33} \mathrm{~s} / \varepsilon_{0}$ & 716 \\
\hline$\kappa_{33}$ & 0.52 \\
\hline $\mathrm{C}_{33}\left(\mathrm{~N} / \mathrm{m}^{2}\right)$ & $16.04 \times 10^{10}$ \\
\hline $\mathrm{A}\left(\mathrm{m}^{2}\right)$ & $386 \times 10^{4}$ \\
\hline \multicolumn{2}{|c|}{ Adhesive } \\
\hline Property (units) & $\begin{array}{c}\text { Epoxy } \\
\text { (Hunstmann) }\end{array}$ \\
\hline$\rho\left(\mathrm{kg} / \mathrm{m}^{3}\right)$ & 1400 \\
\hline$v(\mathrm{~m} / \mathrm{s})$ & 2344 \\
\hline$\alpha_{v}(\mathrm{~Np} / \mathrm{m})^{4}$ & 172 \\
\hline $\mathrm{A}\left(\mathrm{m}^{2}\right)$ & $386 \times 10^{4}$ \\
\hline \multicolumn{2}{|c|}{ Metal } \\
\hline Property (units) & Aluminum \\
\hline$\rho\left(\mathrm{kg} / \mathrm{m}^{3}\right)$ & 2791 \\
\hline$v(\mathrm{~m} / \mathrm{s})$ & 6291 \\
\hline$\alpha_{\mathrm{v}}(\mathrm{Np} / \mathrm{m})^{5}$ & 0.46 \\
\hline $\mathrm{A}\left(\mathrm{m}^{2}\right)$ & $386 \times 10^{4}$ \\
\hline
\end{tabular}

Fig. 4.14 shows the impedance curve from 1 to $4 \mathrm{MHz}$ of the PZT 4 transducer for the two models, Pspice and analytical and experimentally acquired on VNA.

4 The viscous attenuation of the adhesive was acquired from Prada et. al.

5 The viscous attenuation of the Aluminum was acquired from [20] 


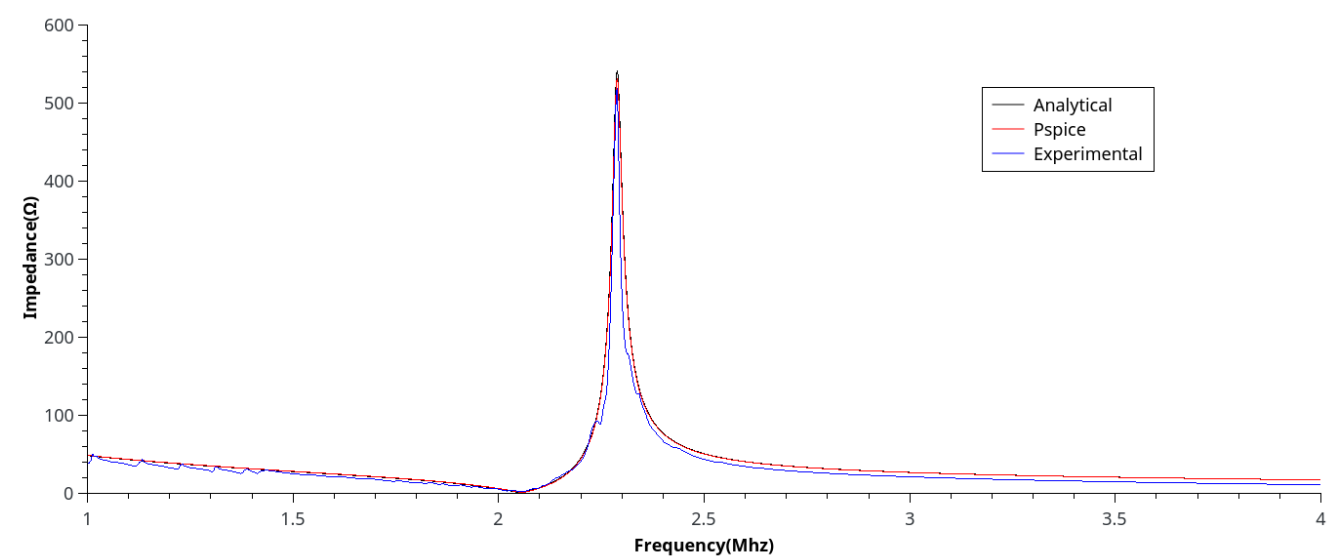

Figure 4.14 Impedance curve for Pspice, Analytical and experimental

\subsubsection{Flat plate power transfer analysis}

A diagram illustrating the power transfer characterization of the flat plate and the experimental characterization using VNA is showed in Fig. 4.15.
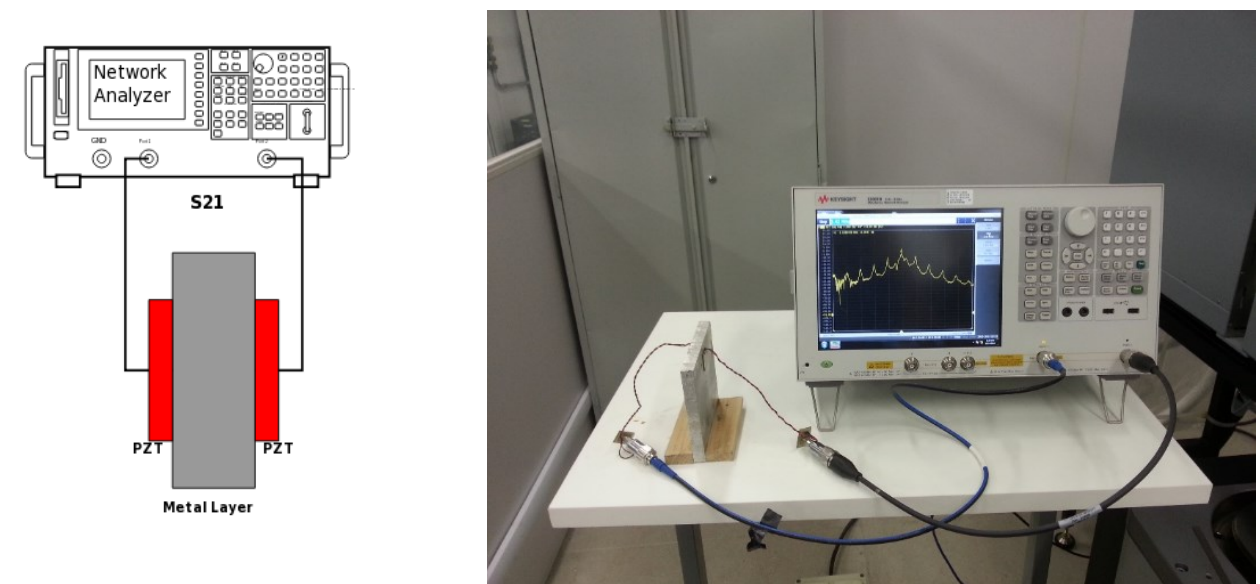

Figure 4.15 Schematic (a) and experimental (b) characterization of the flat plate using VNA

Fig. 4.16 compares the analytical, the Pspice simulated and the experimental power transfer, data is acquired from $1 \mathrm{MHz}$ to $4 \mathrm{MHz}$ with 1601 points. The Pspice maximum point is reached at $2.186 \mathrm{MHz}$ with a insertion loss of $3.23 \mathrm{~dB}$. The maximum frequency of the analytical model is extremely close at $2.188 \mathrm{MHz}$, however with $6.54 \mathrm{~dB}$ of insertion loss, a considerable attenuation comparing to PSPice. Finally, the experimental analysis is also at $2.188 \mathrm{MHz}$ and its insertionloss is $5 \mathrm{~dB}$. Table 4.2 summarizes this values. 


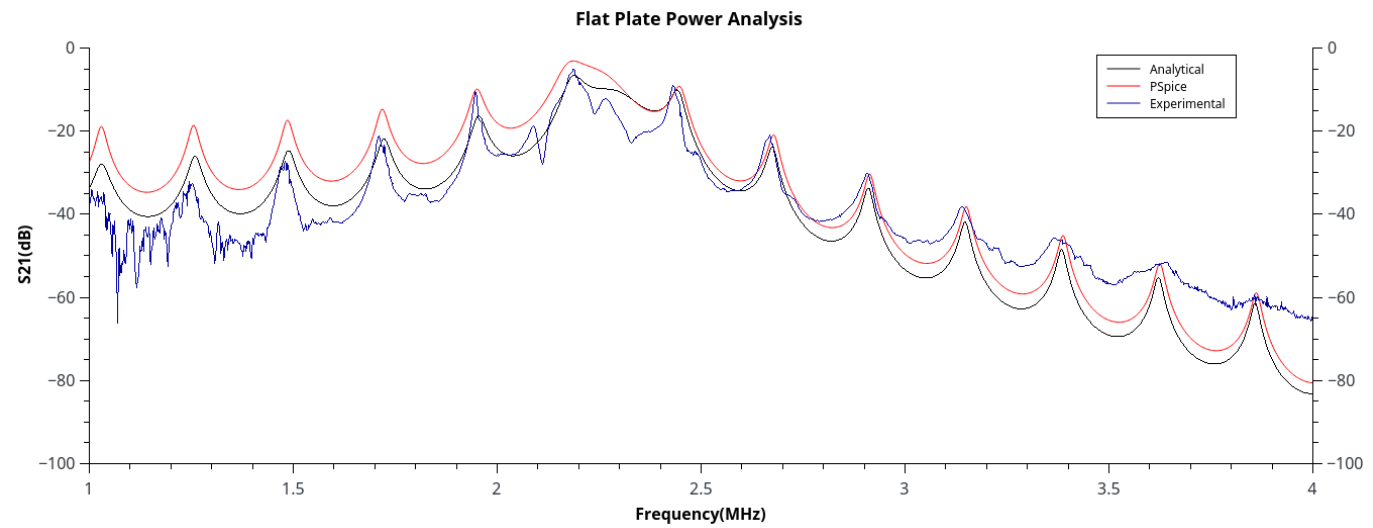

Figure 4.16 Flat plate power analysis

Table 4.2 Power Transfer of Pspice, analytical and experimental

\begin{tabular}{|c||cc||}
\hline \multicolumn{3}{|c|}{ Power Transfer } \\
\hline Method & $\begin{array}{c}\text { Frequency of } \\
\text { maximum } \\
(\mathrm{MHz})\end{array}$ & $\begin{array}{c}\text { Maximun } \\
\text { S21(dB) }\end{array}$ \\
\hline Pspice & 2.186 & -3.23 \\
Analytical & 2.188 & -6.54 \\
Experimental & 2.188 & -5
\end{tabular}

The main difference between Pspice and analytical model is related to the losses implemented in each model, as discussed in the chapter 3. When comparing both models with the experiment, it is observed that the peaks of resonance distributed in the frequency range match. It is also noted that the analytical model has more consistency with the experiment. This suggests that the more strict losses modeled in the analytical implementation are relevant for a more precise characterization of the physical phenomenon.

\subsubsection{2}

\section{Flat plate data communication analysis}

As done to determine the S21 parameter, which is a function of frequency, the communication is firstly analyzed in the frequency domain, as presented in section 3.2.4. The diagram of figure 4.17 shows the setup made to acquire the reffected impedance (Z11) in VNA using the impedance analyzer tool. In this configuration only the port 1 of the VNA is used. The signal generator (ARB) varies the load terminator of inside PZT into $50 \Omega$ and an equivalent impedance of 0.22 
$\Omega$. Then the measured spectrum of Z11 is transformed to V11, which is the main parameter to evaluate data communication. This signal is labeled by number (1) in Fig. 4.8.

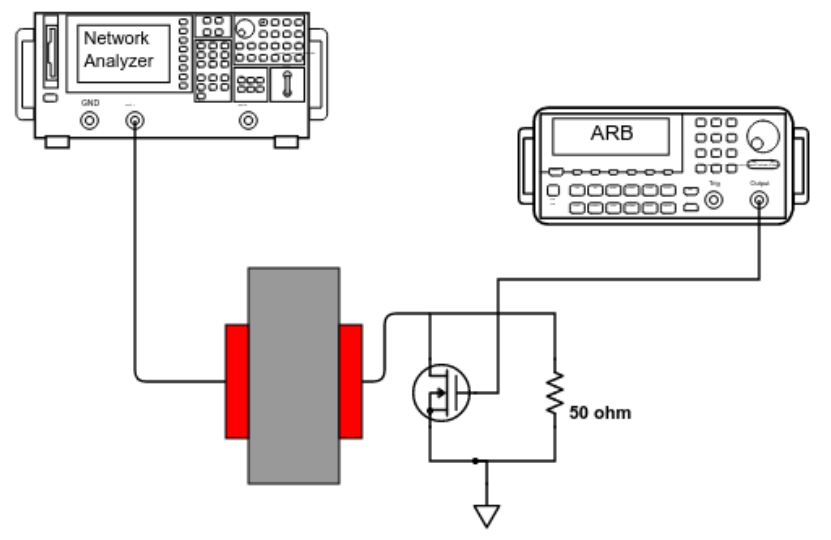

Figure 4.17 Setup for data communication analysisin frequency domain

The relationship between the impedance and the voltage uses the principle of voltage divider. Figure 4.18 illustrates a schematic of the equivalent circuit. Eq. 103 represent the conversion between Z11 and V11 when the output impedance of port 1 is set to $50 \Omega$ and the amplitude of the voltage generator is $1 \mathrm{Vpp}$.

$$
V_{11}=\frac{Z_{11}}{50+Z_{11}}
$$

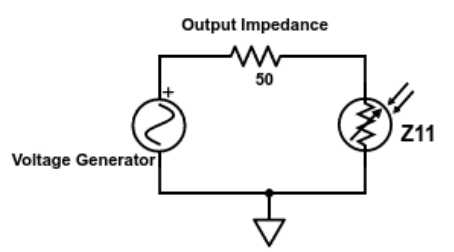

Figure 4.18 Voltage divider theory

The Pspice directly gives the input voltage as it is a circuit simulator, however, the analytical model has to be manipulated to transform the ABCD transfer function into a voltage relationship and acquire the (V11), as explained in section 3.2.6.

Fig. 4.19 shows the input voltage (V11) for the two termination load levels, for Pspice, analytical model and experiment. Fig. 4.20 illustrates the difference in V11 between the two load levels. In both curves a zoom is made in the range that contains the maximum difference in voltage amplitude. 

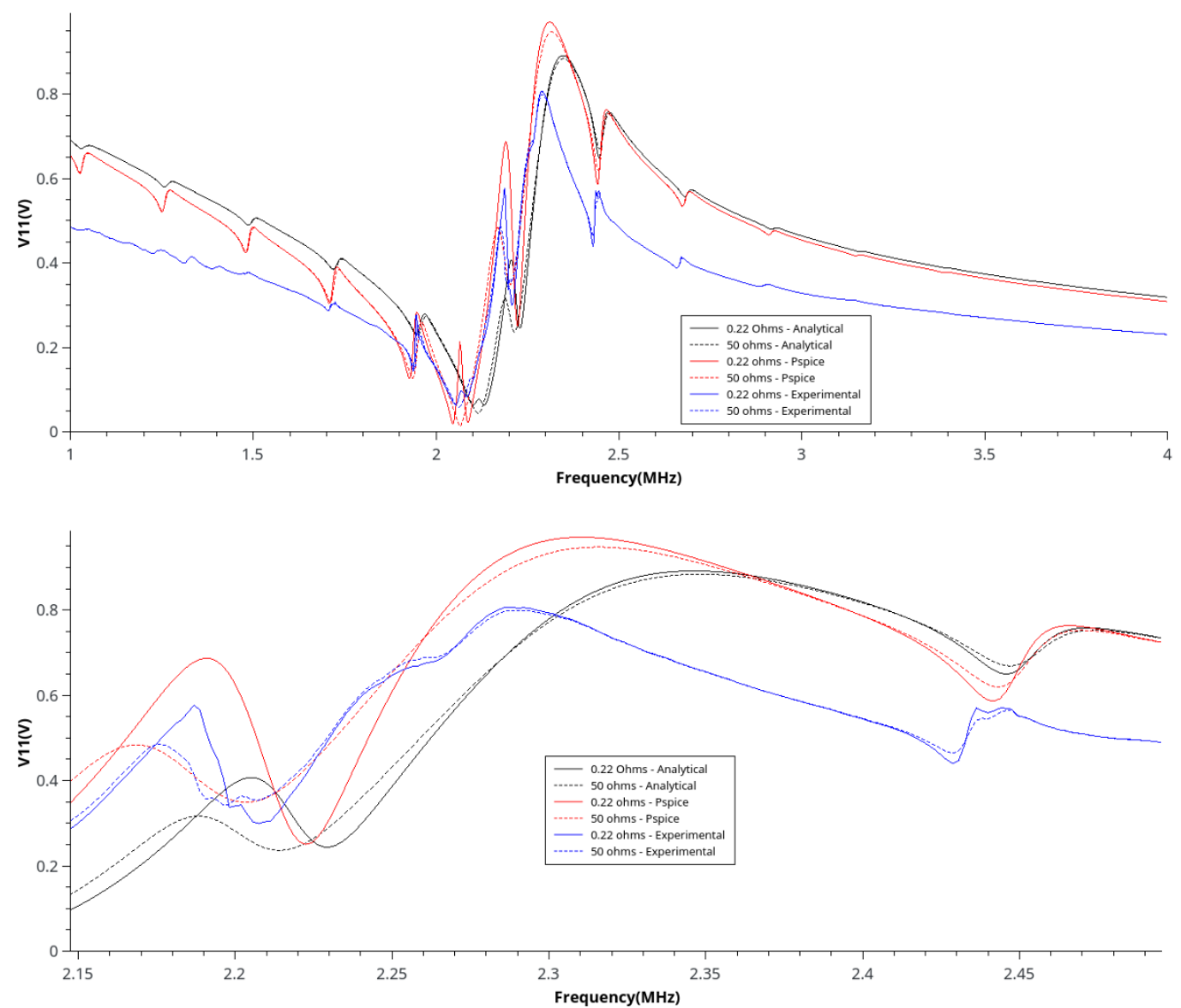

Figure 4.19 (a) Input voltage (V11) varying the termination load and zoom (b) between 2.1 and $2.5 \mathrm{MHz}$

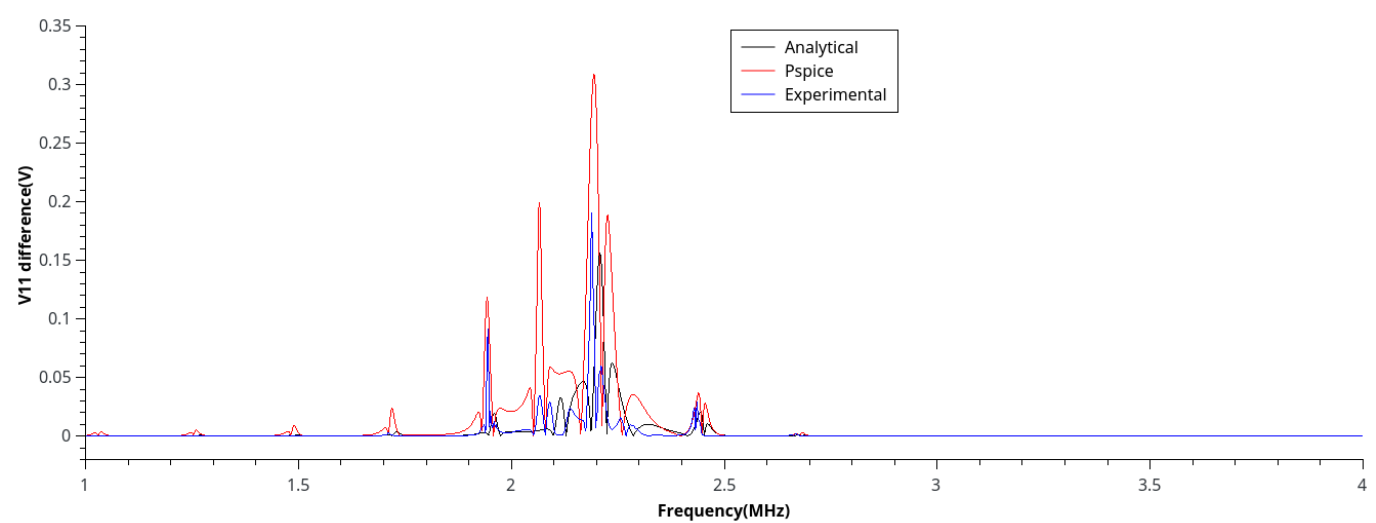

Figure 4.20 Difference in amplitude voltage

In Pspice, the frequency that has the highest difference in V11 is located at 2.194MHz, which is $4 \mathrm{kHz}$ shiffed from the point of maximum power transfer. In this frequency the voltage variation is $0.31 \mathrm{~V}$. For the analytical model the maximum difference in V11 is $0.15 \mathrm{~V}$ at $2.206 \mathrm{MHz}$ The best fequency to communicate the data is slightly distant from the highest point to transfer power in this model, having a shiff of $18 \mathrm{kHz}$. The experimental curve has the same pattern in Pspice and in the 
analytical model. However, the analytical model is more precise in the differences of V11, when compared to the experiment. This is probably due to the associated losses which are frequency dependent, see section 3.2.4. The experiment presented a peak of V11 difference of $0.19 \mathrm{~V}$ at $2.188 \mathrm{MHz}$, which is totally coincident with the point of maximum power transfer. Table 4.3 summarize the maximum voltage difference.

Table 4.3 Highest difference in amplitude

\begin{tabular}{||c||cc||}
\hline \multicolumn{3}{|c|}{ Maximum voltage difference } \\
\hline Method & Frequency (MHz) & V11 difference (V) \\
\hline Pspice & 2.194 & 0.31 \\
Analytical & 2.206 & 0.15 \\
Experimental & 2.188 & 0.19
\end{tabular}

In the sequel, the flat plate is exposed to a time domain analysis that is based on the diagram of Fig. 4.21. In this figure, the output signal with a constant frequency is connected to the outside PZT. On the other side the inside PZT is switched to ground with a MOSFET.

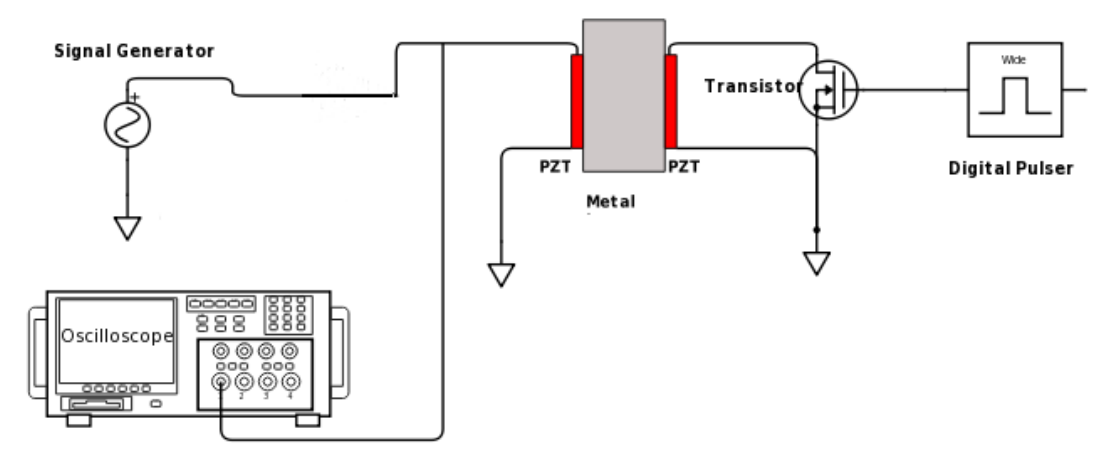

Figure 4.21 Setup to measure the input voltage (V11)

The resulting signal seen in the oscilloscope is shown in figure 4.22. The signal generator amplitude was set to $1 \mathrm{~V}$ at $2.188 \mathrm{MHz}$. The variation of amplitude considering the maximum and minimum amplitude of each period of the digital pulser is approximately $0.16 \mathrm{~V}$. This figure also shows the signal obtained by Pspice operating with $1 \mathrm{~V}$ at $2.194 \mathrm{MHz}$. About $0.3 \mathrm{~V}$ of difference in amplitude between high and low levels was obtained. Both values are coherent with the ones of table 4.3 and in both case the high level occur when the MOSFET is conducting and the low level when the terminator is connected to a $50 \Omega$ termination load. This analysis 
was not performed with the analytical model because this is based only on frequency domain with harmonic responses, as commented in section 3.2.6.

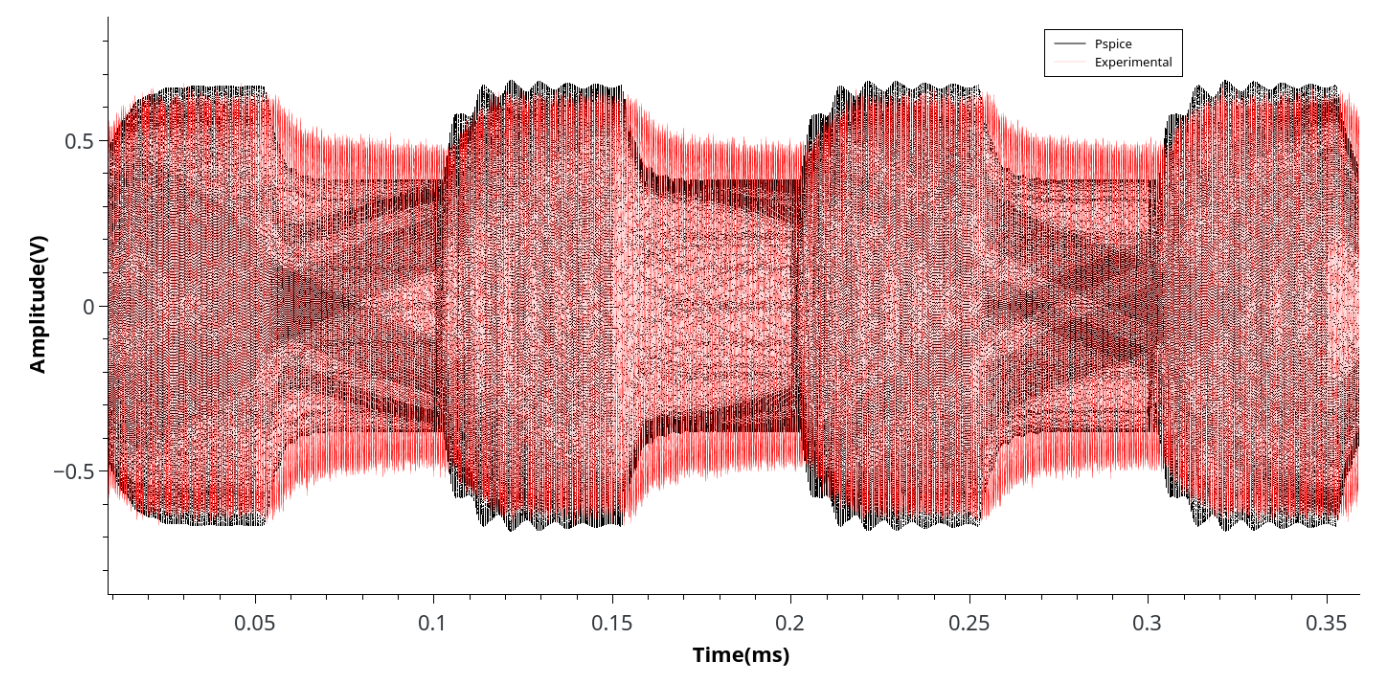

Figure 4.22 Input voltage signal flat plate experimental test

The result of $0.16 \mathrm{~V}$ in amplitude difference makes the demodulation quite challenging using the elements presented here, mainly because of the threshold voltage noise of the comparator. However amplifying the signal 10 times, for example, and considering a linear system, the amplitude difference could increase also 10 times, keeping the threshold voltage noise constant and turning the demodulation much easier

Analyzing the behavior of the signal it is possible to conclude that the multipath propagation of PDAC turns the acoustic channel into a resonant system when operating at a specific frequency. Consequently, the multiple propagating waves create a standing wave inside the material. Every time the input PZT is switched, a new standing wave has to be established. In practice, this phenomenon can be observed in Fig. 4.22. Until the steady state is reached the signal has a time response when varying between the two state levels. 


\subsection{2}

\section{Curved surface - Tube}

A second experiment was carried out, it consists oftwo concentric tubes. One tube is made of carbon steel and has $325 \mathrm{~mm}$ of external diameter and $6.5 \mathrm{~mm}$ of thickness, the second is made of aluminum and has $220 \mathrm{~mm}$ of external diameter. The space between the tubes was filled with water. The water layer has $67.8 \mathrm{~mm}$. The table 4.2 summarizes the properties of the additional materials used in this second experiment. Properties that does not have the footnote were characterized using the pulse-echo method, scale and micrometer.

Table 4.4 Material properties - Tube

\begin{tabular}{|c|c|}
\hline \multicolumn{2}{|c|}{ Piezoelectric } \\
\hline Property (units) & $\begin{array}{c}\text { PZT4 } \\
\text { (Ultraceram) }\end{array}$ \\
\hline$\rho\left(\mathrm{kg} / \mathrm{m}^{3}\right)^{\circ}$ & 7900 \\
\hline$v(\mathrm{~m} / \mathrm{s})$ & 4671 \\
\hline $\mathrm{Qm}$ & 234 \\
\hline$\varepsilon_{33} \mathrm{~s}^{\circ} \varepsilon_{0}$ & 889 \\
\hline$\kappa_{33}$ & 0.57 \\
\hline $\mathrm{C}_{33}\left(\mathrm{~N}^{2} \mathrm{~m}^{2}\right)$ & $17.2 \times 10^{10}$ \\
\hline $\mathrm{A}\left(\mathrm{m}^{2}\right)$ & $491 \times 10^{4}$ \\
\hline \hline \multicolumn{2}{|c|}{ Metal } \\
\hline Property $(\mathrm{units})$ & Carbon Steel \\
\hline$\rho\left(\mathrm{kg} / \mathrm{m}^{3}\right)$ & 7715 \\
\hline$v(\mathrm{~m} / \mathrm{s})$ & 5874 \\
\hline$\alpha_{\mathrm{v}}(\mathrm{Np} / \mathrm{m})$ & 0.8 \\
\hline $\mathrm{A}\left(\mathrm{m}^{2}\right)$ & $491 \times 10^{4}$ \\
\hline \hline \multicolumn{2}{|c|}{ Fluid } \\
\hline Property $(\mathrm{units})$ & Water $\left(20^{\circ} \mathrm{C}\right)$ \\
\hline$\rho\left(\mathrm{kg} / \mathrm{m}^{3}\right)^{\prime}$ & 1000 \\
\hline$v(\mathrm{~m} / \mathrm{s})$ & 1484 \\
\hline$\alpha_{\mathrm{v}}(\mathrm{Np} / \mathrm{m})^{\circ}$ & 0 \\
\hline $\mathrm{A}\left(\mathrm{m}^{2}\right)$ & $491 \times 10^{4}$ \\
\hline \multicolumn{2}{|c|}{} \\
\hline
\end{tabular}

6 Data taken from manufacture contact

7 http://www. ondacorp.com/images/Liquids.pdf

8 The viscous loss is $0.001 \mathrm{~Np} / \mathrm{m} @ 400 \mathrm{Khz}$ in [108] 
The water layer is simulated as an intermediate layer in Pspice and analytical models. The viscous loss of water is extremely low and for this reason it can be considerate $0 \mathrm{~Np} / \mathrm{m}$ [108] however, considering the thickness of intermediate layer the spreading loss must be calculated. A three-dimensional sketch of this experimental configuration can be seen in Fig. 4.23. For this experiment two PZT4 transducers with nominal frequency of $1 \mathrm{MHz}, 2 \mathrm{~mm}$ of thickness, purchased from UltraCeram ${ }^{\circledR}[109]$ is used. The external PZT is bonded on the external surface of the tube with Hunstmann ${ }^{\circledR} 2015$ epoxy. Before this, a recess is made on the curved surface of the external tube leaving a plane surface area that facilitates the bonding.

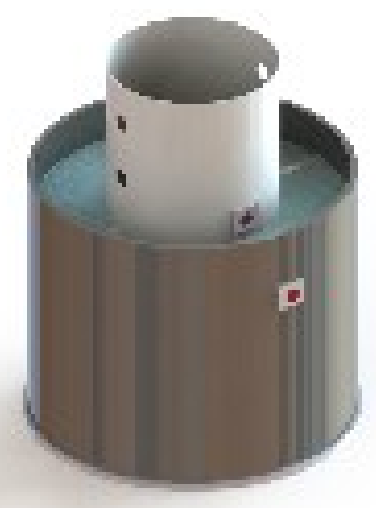

Figure 4.23 Three-dimensional sketch of curved surface experiment

On the other hand, the internal PZT was attached with the aid of a structure made on a 3D printer, model Replicator Z18 by MakerBot ${ }^{\circledR}$ and illustrated in Fig. 4.24. This structure allows the alignment between the internal PZT and the external PZT and isolates the transducer back face from the water, (front face illustrated in Fig. 4.24) this ensures that the transducer back layer is filled with air. The presence of air on the transducer back layer is important so the waves on the back face of the transducer does not interact with the materials in the back. An air layer introduces a very poor coupling between the ceramics and the material due to the high impedance mismatch between ceramic and air. Another purpose of the back layer filled with air is to minimize the bandwidth of the transducer keeping the Q fact or as high as is possible [110]. 


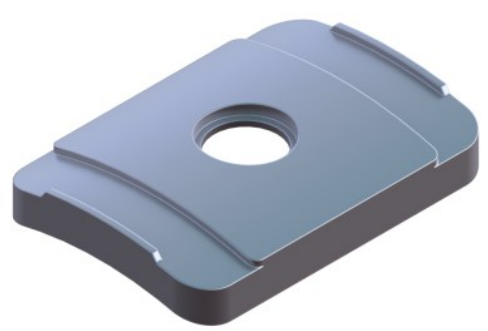

Figure 4.24 3D CAD and Structure made on 3D printer to isolate the back face of transducer from water

\subsubsection{1}

\section{Curved Surface power transfer analysis}

As done for the ffat plate experiment a comparison of the power transfer is made between the two models and the tube experiment using VNA, which is calibrated and set to realize measurements in the range of $800 \mathrm{kHz}$ tol. $4 \mathrm{MHz}$ with 1601 points. The Fig 4.25 shows the three curves.

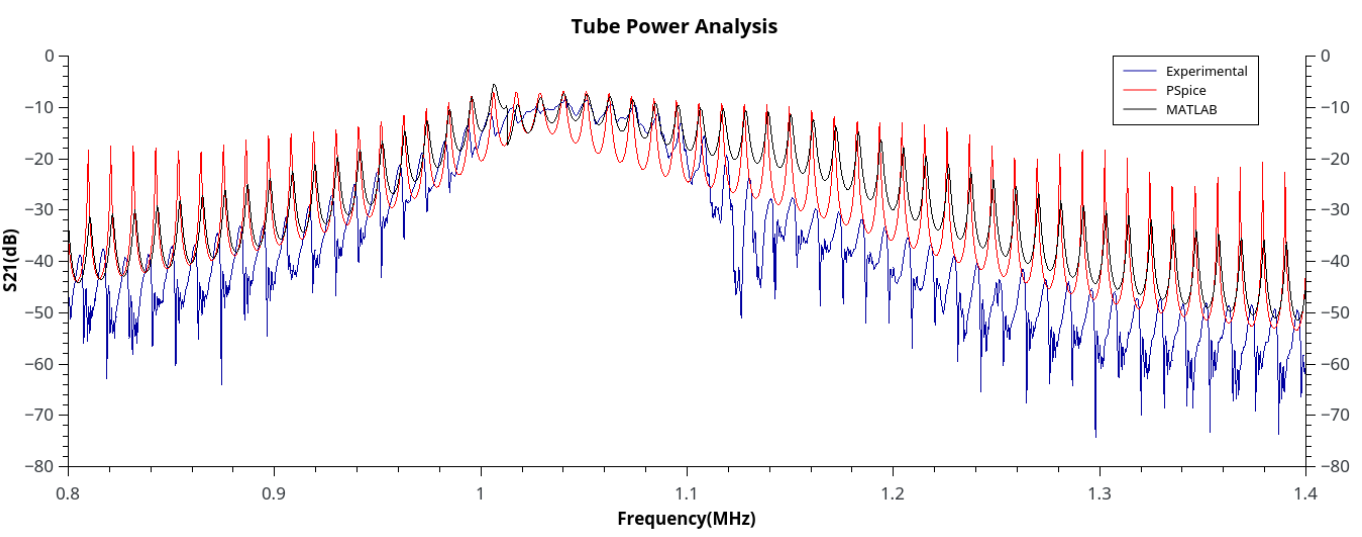

Figure 4.25 Power transfer analysis for curved surface experiment

One can notice the coincident peaks of resonance between the Pspice and analytical model but slightly difference, approximately $4 \mathrm{kHz}$, when comparing with the experimental. This can be caused by distinct characterized values of the many necessary parameters, see table 4.4 , and the one-dimensional model implemented. Anyhow, the peak of transmission has $6.86 \mathrm{~dB}$ at $1.040 \mathrm{MHz}$ of insertion loss for Pspice model, 7.41dB for analytical model at $1.041 \mathrm{MHz}$ and 8.45 at $1.030 \mathrm{MHz}$ and $1.043 \mathrm{MHz}$ for experimental. Table 4.5 summarizes the insertion loss in each case. 
Table 4.5 Power transfer peak of curved surface experimental setup

\begin{tabular}{|c|c|c|}
\hline \multicolumn{3}{|c|}{ Power Transfer peak } \\
\hline Method & Frequency(MHz) & $\mathrm{S} 21(\mathrm{~dB})$ \\
\hline Pspice & 1.040 & -6.86 \\
\hline Analytical & 1.041 & -7.41 \\
\hline Experimental & $1.030 / 1.043$ & -8.45 \\
\hline
\end{tabular}

Similar to the ffat plate case, the Pspice model has the resonance peaks with low insertion loss for all the spectrum and the analytical more coincident with the experimental, mainly for the range between $800 \mathrm{kHz}$ to $1.04 \mathrm{MHz}$.

\subsubsection{2}

\section{Curved Surface data communication analysis}

Following the power transfer analysis, the data communication analysis is made in Pspice, for the analytical model as well as for the experiment setup, setting the range of analysis to $800 \mathrm{kHz}$ to $1.4 \mathrm{MHz}$ with 1601 points, Figure 4.26 shows the curve of the input voltage (V11) for Pspice, analytical and experiment varying the load termination between $50 \Omega$ and $0.22 \Omega$.

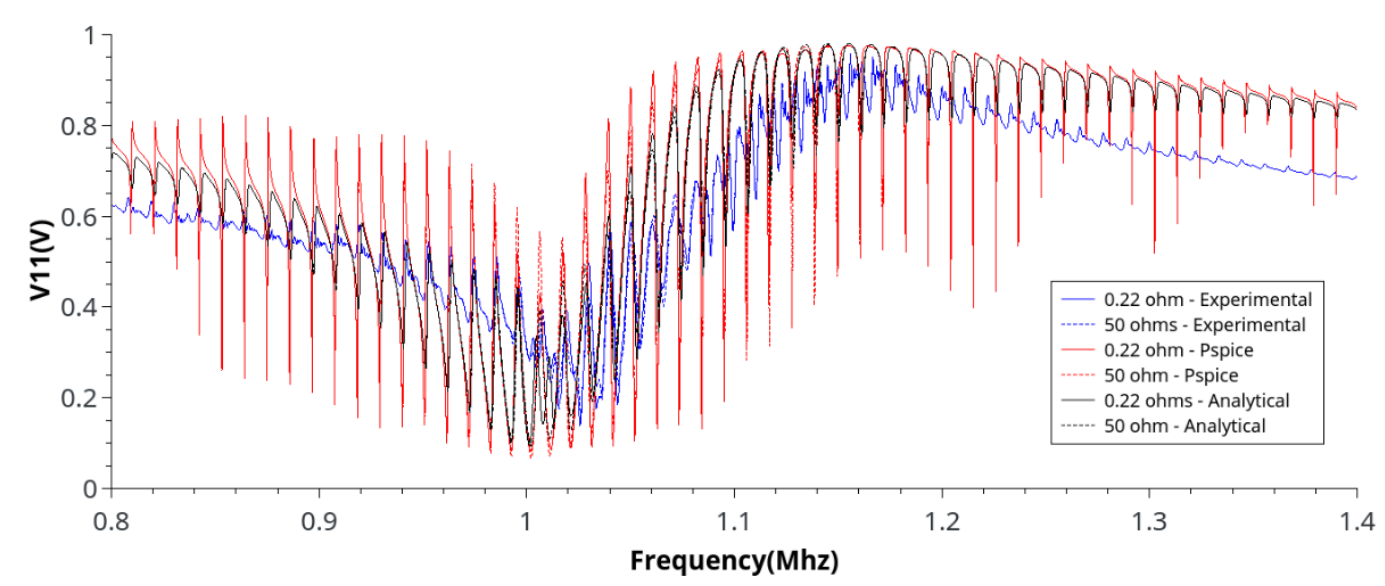

Figure 4.26 Input voltage (V11) - curved surface

The difference between these three curves of amplitude is best seen in the Fig. 4.27. The figure shows the range $995 \mathrm{kHz}$ to $1.05 \mathrm{MHz}$. Although, the analysis of power transfer shows that the Pspice and analytical model are very similar, the analysis of the input voltage shows differently. One can notice in Fig. 4.27 that the amplitude of the analytical model is closer to the experimental data. 


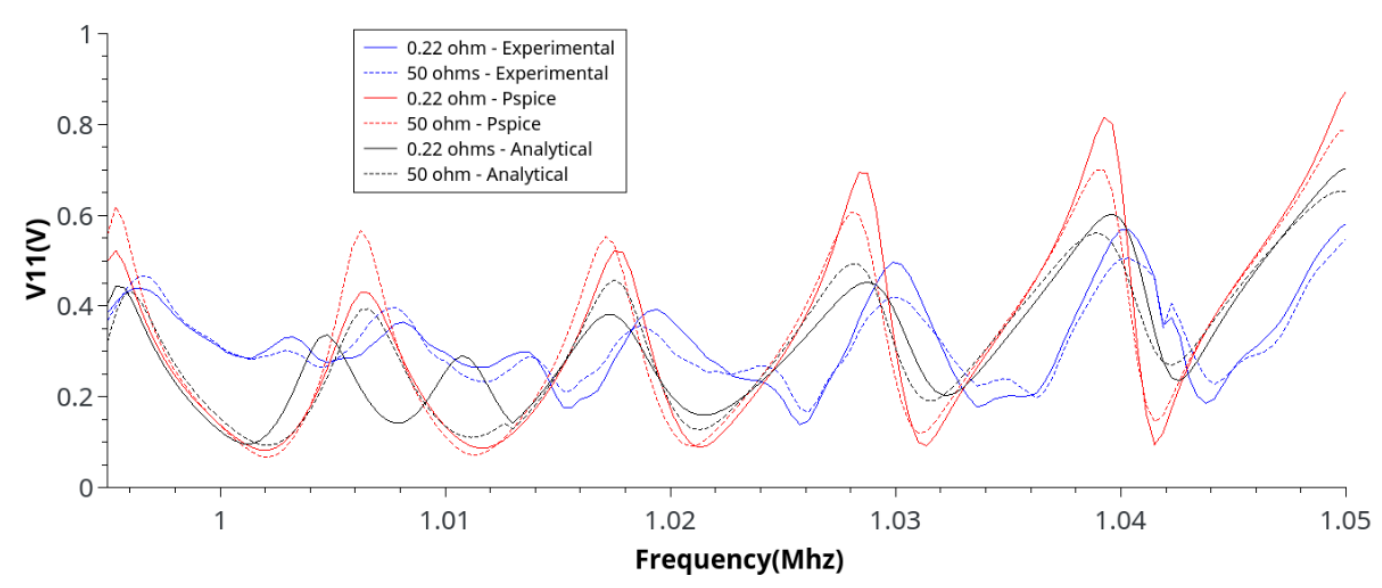

Figure 4.27 Input voltage between $0.95 \mathrm{MHz}$ and $1.05 \mathrm{MHz}$ - curved surface

It is possible to notice from Fig. 4.27 that in Pspice the frequency which has the maximum difference in voltage $(1.030 \mathrm{MHz})$ is considerable different from the maximum power transfer $(1.040 \mathrm{MHz})$, however a second peak with high amplitude is located at $1.040 \mathrm{MHz}$. The analytical model also has a difference between the maximum power transfer $(1.041 \mathrm{MHz})$ and the maximum difference in voltage $(1.008 \mathrm{MHz})$ but also has a high peak at $1.041 \mathrm{MHz}$. The experimental, otherwise, has the two major peaks, with almost the same level, which are coincident for the maximum power transfer and difference in voltage $(1.030 \mathrm{MHz}$ and $1.043 \mathrm{MHz})$. Table 4.6 summarizes the results of V11 difference for the Fig. 4.27.

Table 4.6 Voltage difference of the input voltage

\begin{tabular}{|c||c||c||}
\hline \multicolumn{3}{|c|}{ Maximum voltage difference } \\
\hline Method & $\begin{array}{c}\text { Frequency } \\
(\mathrm{MHz})\end{array}$ & $\begin{array}{c}\text { V11 difference } \\
(\mathrm{V})\end{array}$ \\
\hline Pspice & 1.030 & 0.1629 \\
Analytical & 1.0081 & 0.17 \\
Experimental & $1.030 / 1.043$ & 0.07
\end{tabular}

Comparing this result with that the one shown in Fig. 19b, it is observable an increase in the number of peaks since this experiment has a water layer with high thickness $(74.32 \mathrm{~mm}$ instead of $13.08 \mathrm{~mm})$. The system with a higher thickness and lower vel ocity has higher selectivity in fequency. This selectivity al so known as cyclic frequencies [7] happens when a standing wave is formed in the layers of the acoustic channel and it is govemed by the Eq (104). 


$$
f_{n}=\frac{n * c_{m}}{2 l}
$$

Where $c_{m}$ is the metal sound velocity and 1 is the thickness of metal [7].

Nevertheless, the difference in voltage(V11) is proportional to the losses and the matching between the acoustic impedances of the materials. With higher thickness and more layers, the difference in V11 has lower voltages when comparing with the flat plate experiment, however with a higher thickness and/or lower velocity the system increases the amount of standing waves per frequency interval formed inside the layers and more frequencies appear as candidates to be used. One can also notice that the values of the difference in V11 illustrated in Fig. 4.29 has almost the same peaks amplitude for this range.

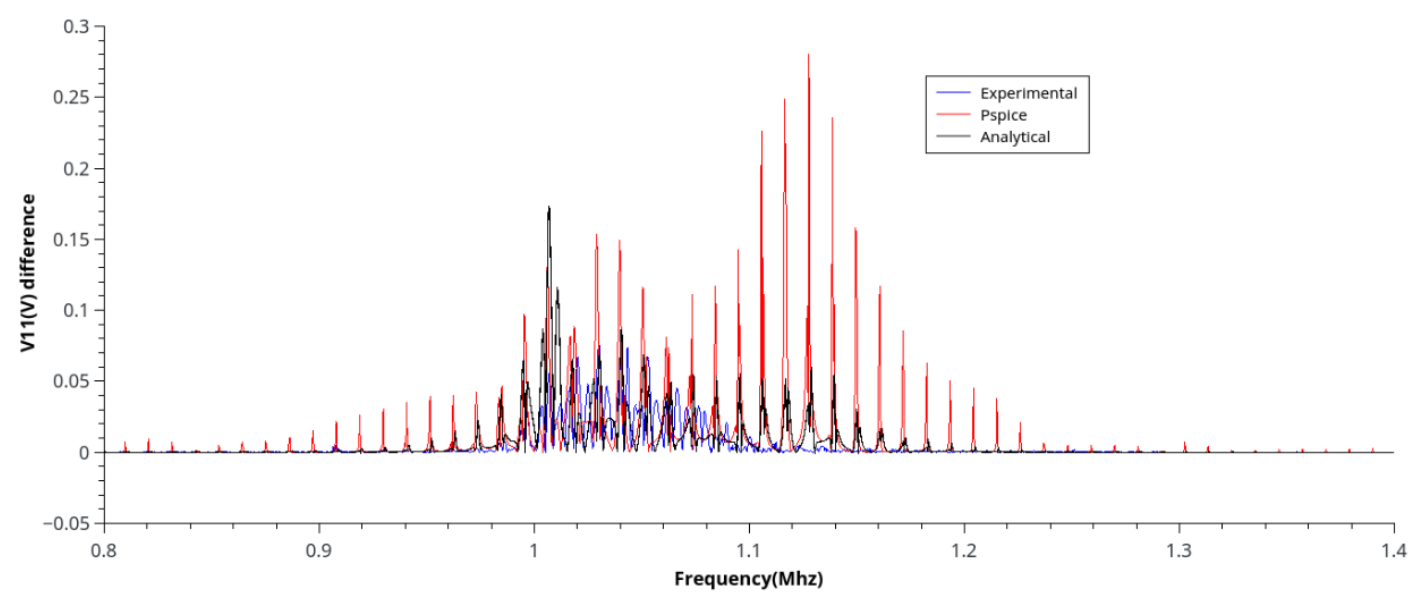

Figure 4.28 Input voltage difference between 50 and $0.22 \mathrm{ohms}$, full frequency range

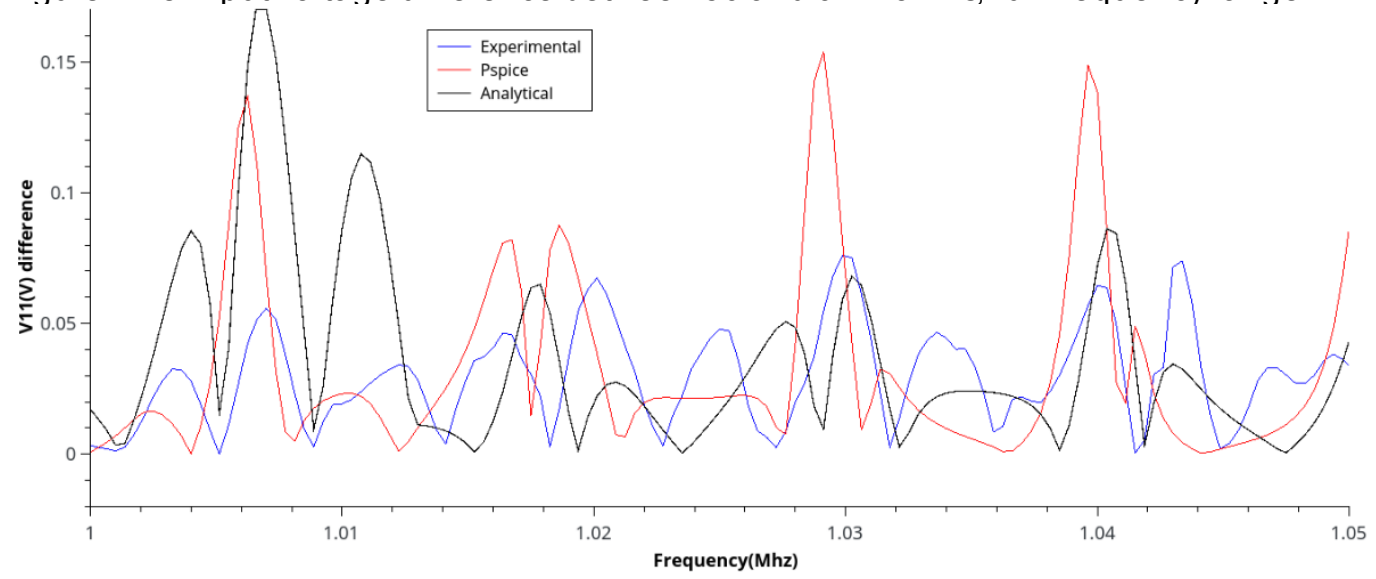

Figure 4.29 Input voltage zoom for frequencies between $1 \mathrm{MHz}$ and $1.05 \mathrm{MHz}$ 
Comparing the frequency in which the maximum difference in V11 (Table 4.6 and 4.3, respectively) and the maximum power transfer (Table 4.5 and 4.2, respectively) occur for both, in fat plate and curved surface cases, it reveals a small and medium differences between these frequency points in Pspice and analytical models, respectively. Nevertheless, the experimental results shows that for both, ffat plate and curved surface, the frequency that has the maximum difference in V11 and power transfer are coincident.

Fig. 4.30 represents the transient analysis of V11 for experimental and Pspice model. In this analysis the frequency of the signal generator is set to operate at 1.040Mhz with a output of $10 \mathrm{~V}$, for both experimental and Pspice simulation. The same frequency is used for Pspice transient analysis simulation. The maximum difference in voltage for Pspice is $1.5 \mathrm{~V}$ and $0.6 \mathrm{~V}$ for the experimental setup. At this frequency the values have approximately the same values of the frequency domain analysis illustrated in Fig. 4.29 and summarized in Table 6.

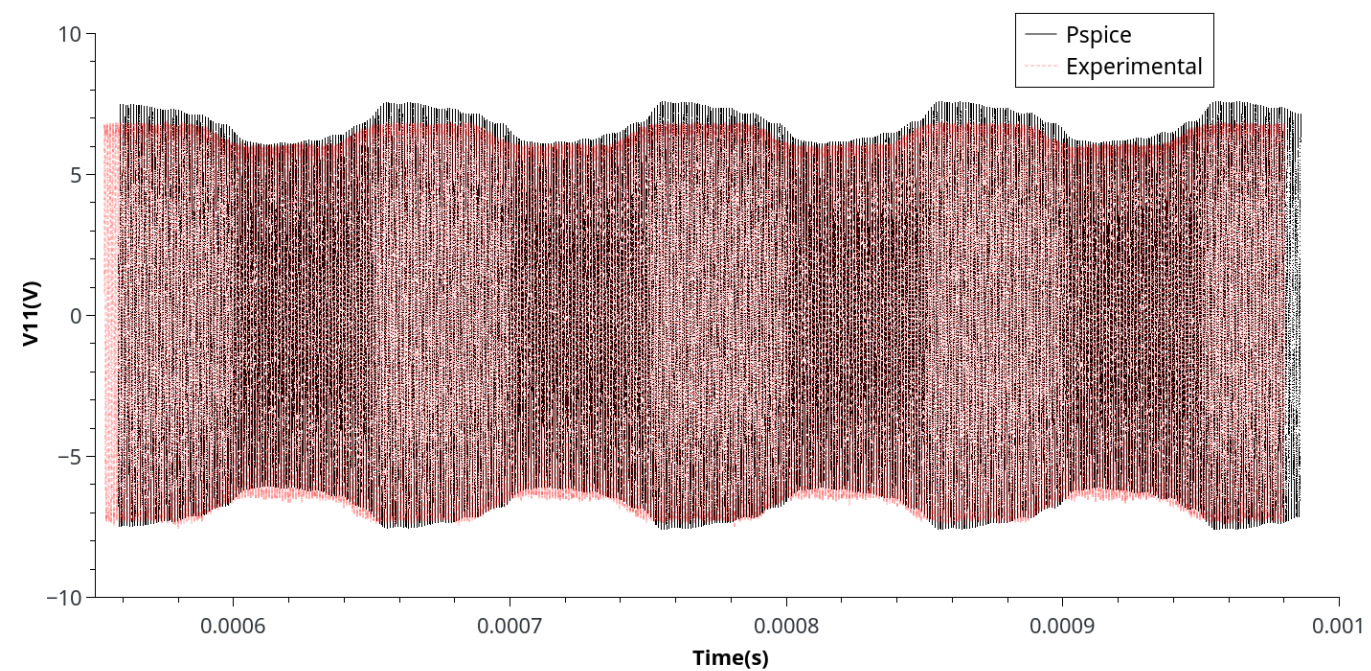

Figure 4.30 Input voltage transient analysis - Curved surface

\subsection{3}

\section{Full system experimental analysis - Tube}

In this section, a full system interrogation with the same configuration of figure 4.9 is detailed using a P\&T sensor connected to the inside module. This full system is assessed in the Curved Surface configuration (section 4.6.2). For the sake of clarity a concise recall of its main blocks is shows in Figure. 4.31. 


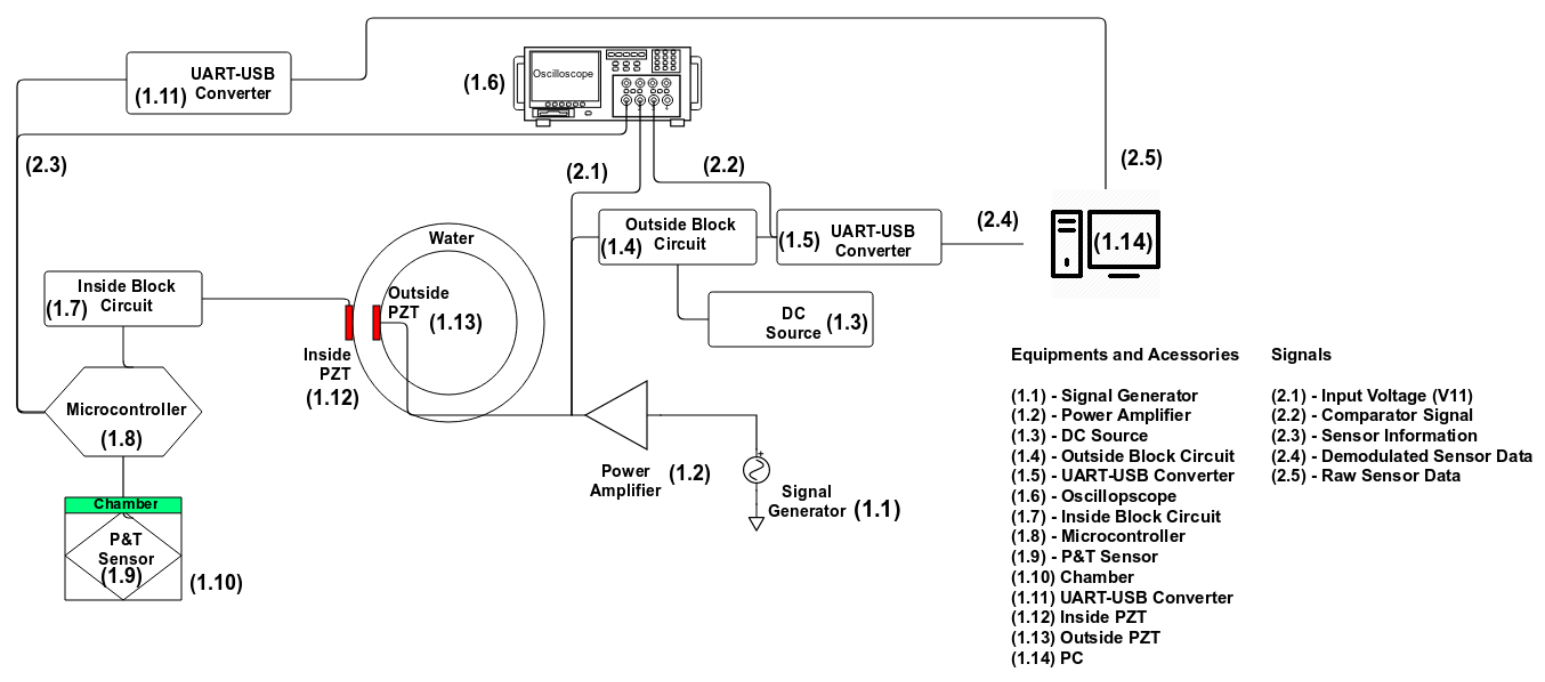

Figure 4.31 Schematic diagram of the curved surface experiment test

As one can see, a fixed frequency is generated by a RF signal generator (label 1.1 in Fig.4.31) and them amplified by an RF amplifer (label 1.2 in Fig.4.31). The outside modules responsible to send the amplified signal to outside PZT transducer (label 1.13 in Fig.4.31) and to send the demodulated sensor data to a personal computer (label 1.14 in Fig.4.31). In this setup the outside block presents the following equipment's: signal generator Tektronix TSG 4104A ${ }^{\circledR}[114]$, (label 1.6 in Fig 4.31), power amplifier $\mathrm{E} \& \mathrm{I} 囚$ model 2100L [115], (label 1.2 in Fig 4.31), and DC source Keythley ${ }^{\circledR}$ model 2220-30-1 [116] , (label 1.3 in Fig 4.31). In the inside block, the P\&T sensor is based on MEMS technology from OpenField ${ }^{\circledR}$ [117], (label 1.9 in Fig 4.31), the microcontroller, MSP430 architecture [118], (label 1.8 in Fig 4.31), has low power feature to ensure the proper functionality of the system since the inside block receives a limited power from the outside block. The metal, PZT s and the adhesive are the same listed on the section 4.6.1. The experimental bench of the full system is shown in the picture of figure 4.32 . The inside and outside block circuits are in metal boxes to minimize the influence of electromagnetic interference. The sensor's data are displayed on the monitor of the PC. The first column is the pressure in (psi) and the second the temperature in $\left({ }^{\circ} \mathrm{C}\right)$. All the cables have $50 \Omega$ of impedance. 


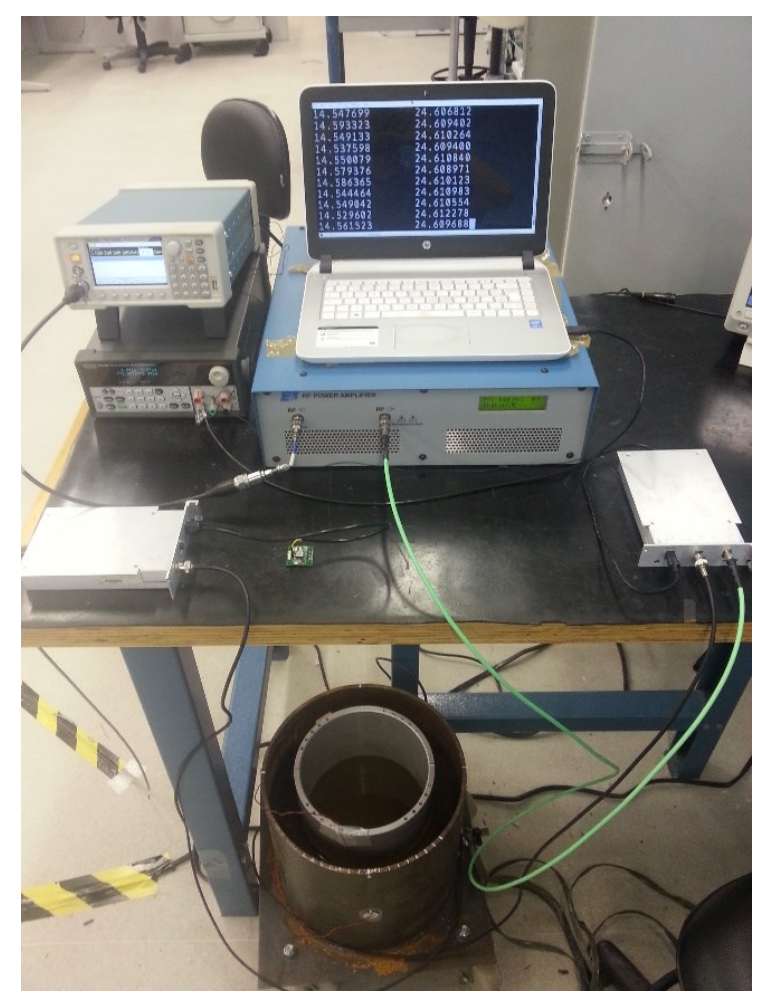

Figure 4.32 Full system experimental bench

Figure 4.33 shows the V11 signal in purple and the sensor information in green, read at the output of the power amplifier, label number (2.1) and at the microcontroller output, label number (2.3) in Fig 4.31, respectively, when the full system is operating. As it can be seen, the high level (1) and the low level (0) of the sensor information has the same "bit" duration of V11 signal, purple signal. The operational frequency is fixed in $1.043 \mathrm{Mhz}$, which provided the best difference in voltage, see section 4.6.2.2, the amplifier output is set to $10 \mathrm{~W}(40 \mathrm{dBm})$ providing approximately $1.4 \mathrm{~W}(31.5 \mathrm{dBm})$ to the inside block, that is, the loss is $8.5 \mathrm{~dB}$ which is the same obtained in section 4.6.2.1. 


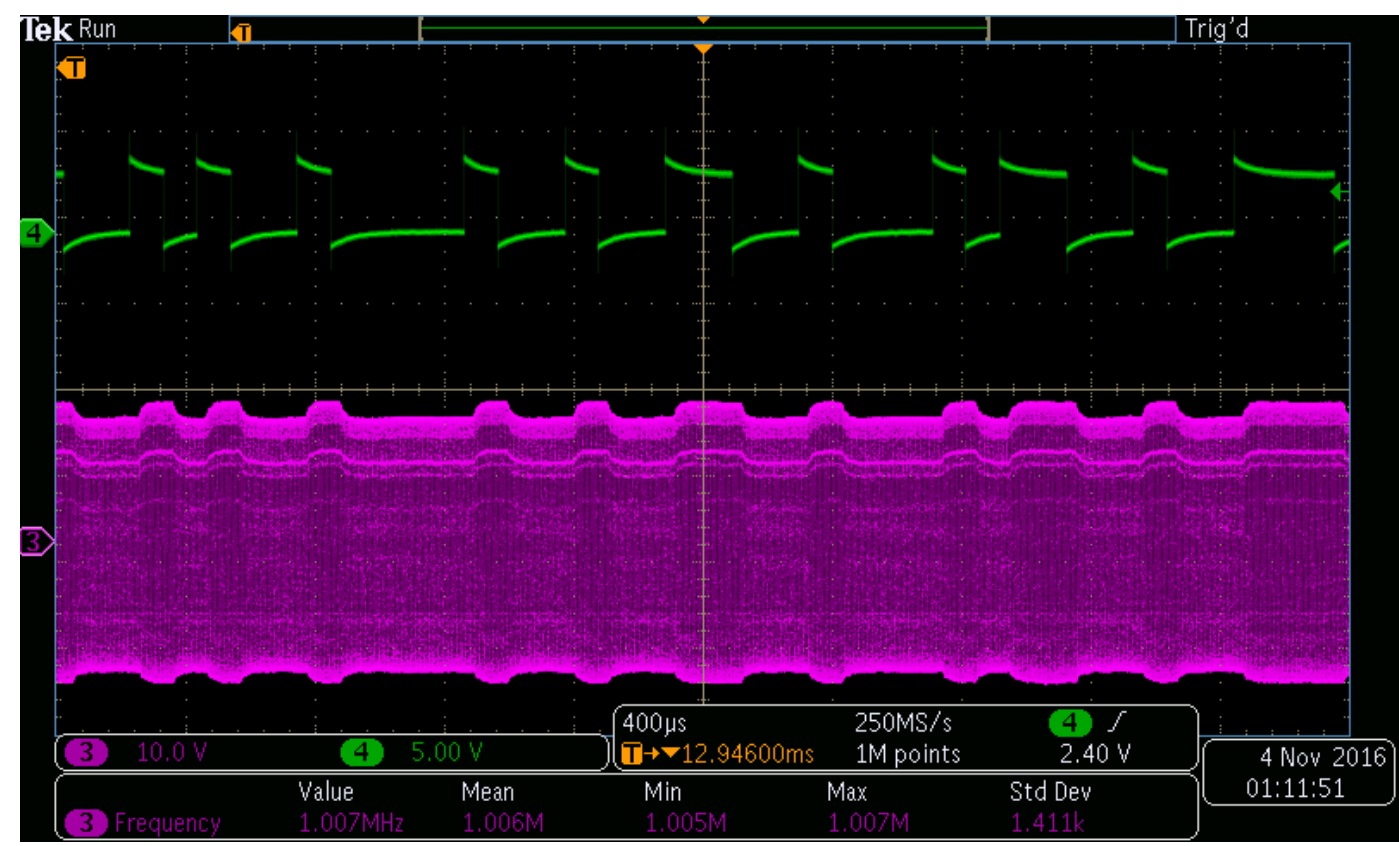

Figure 4.33 Sensor information signal in green and the input voltage signal on the outside block in purple

Table 4.7 Power consumption of the main elementsin the inside block

\begin{tabular}{||c||c|}
\hline \multicolumn{2}{|c|}{ Inside Block Consumption } \\
\hline Element & Power(mW) \\
\hline Microcontroller & 3.3
\end{tabular}

Sensor $\quad 16$

Driver 115

The communication between the microcontroller and the sensor is made using synchronous $\mathrm{I}^{2} \mathrm{C}$ protocol [111], the sensor is interrogated at a rate of $1 \mathrm{~Hz}$ The pressure and temperature values of the sensor are converted to ASCII before transmit ting the information to the outside block using UART. The microcontroller, on the other hand, swit ches the MOSFET through a bipol ar transist or, highlighted by dark green rectangle in Fig. 4.8, as the microcontroller out put port can drive a s the signal up to $4 \mathrm{~mA}$. A diagram, Fig. 4.34, is shown to clarify the sensormicrocontroller communication and switch is operation. 


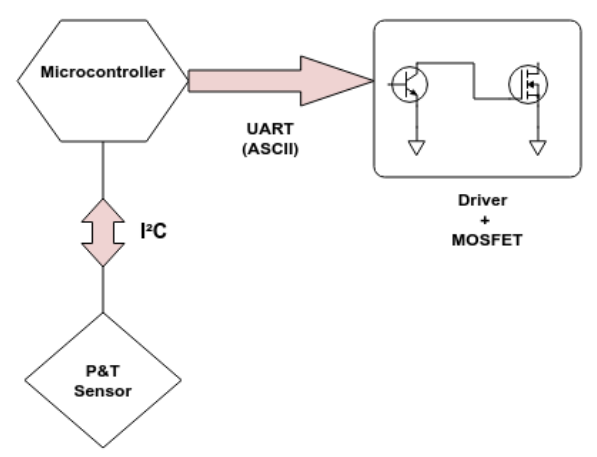

Figure 4.34 Block diagram of protocols used in the inside block

Since UART protocol stays idle with high level (3.3V), the (BJT) driver is configured to invert the digital information of the sensor, switching the 1's by 0 's and vice-versa. Thus, the MOSFET does not short- circuit the inside PZT terminals when not transmitting the information.

The input voltage V1 1 signal passes to the envel ope detect or, label number (2) in Fig. 4.8, and then to the voltage comparator, label number (3) in Fig 4.9. The out put signal, after passing through this two elements is shown in Fig. 4.35 where the purple signal is the demodulated signal and the green the sensor infrmation. One can notice the shift intime between both signals that occur due to wave propagat ion, as commented in section 4.5.2.

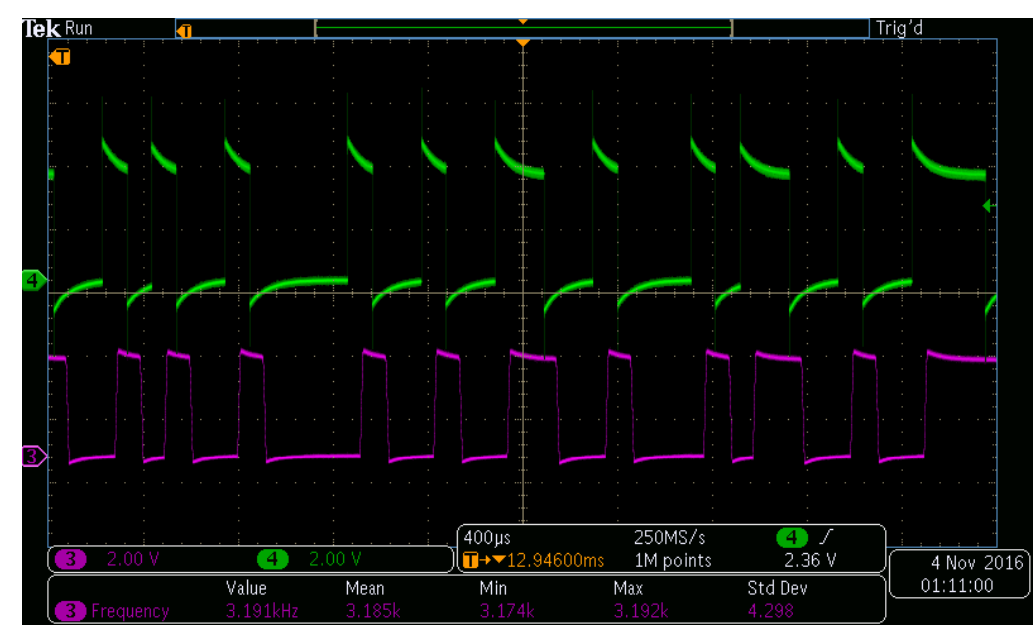

Figure 4.35 Sensor information signal in green and purple the signal after the voltage comparator in purple

The output of the voltage comparator is connected to a computer by using an UART -to-USB converter from FTDI®[119], label number (1.5) in Fig 4.31. Since the sensor's data is in ASCII format, the temperature and pressure values are 
directly displayed in any terminal soffware opening a virtual COM port, as shown in figure 4.32 .

The sensor data passing through the acoustic channel and directed connect to microcontroller is then compared, as shown by flags, label number (2.2) and (2.3), respectively, in Fig 4.31, both ofthem are connected to an UART -to-USB converter before displaying in terminal on PC, as shown by labels (1.5) and (1.11), respectively. Figure 4.36 shows the two curves of temperature acquired in time. The red line stands for the raw sensor data, which is the sensor information in the inside block, black line stands for the demodulated sensor data, and which is the sensor information passing through the acoustic channel on the outside block.

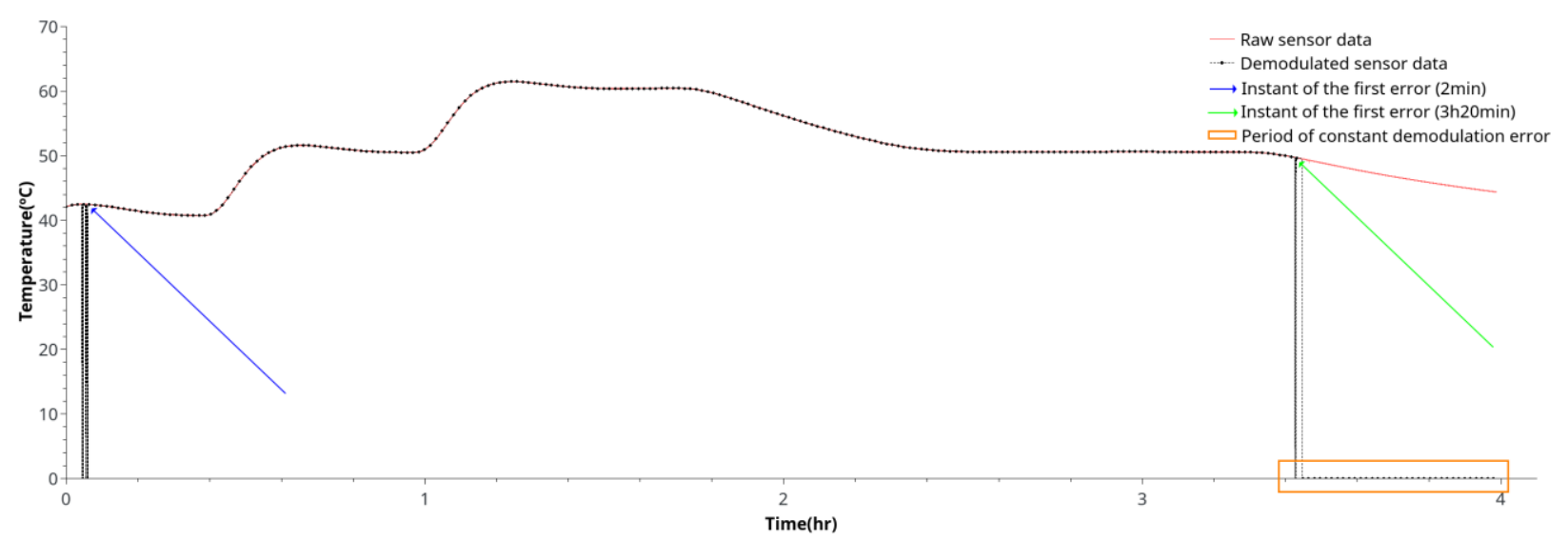

Figure 4.36 Comparison between raw and demodulated temperature sen sor data

The sensor is exposed to temperatures varying from $40^{\circ} \mathrm{C}$ to $60^{\circ} \mathrm{C}$ inside a chamber model 400-TD from EthikT echnology ${ }^{\circledR}$ [120], label number (1.10) in Fig. 4.31. In this test when the system is in the beginning of operation, until the instant represented by the blue arrow in Fig.4.36, the demodulated sensor data reconstruct the information without any errors (one can see that black and red line coincide in the very first instants). However, at approximately two minutes later, the amplitude of the signal after the envelope detector, label number (2), in Fig. 4.8 starts to decrease and the reference (threshold level) of the comparator is out of the high and low levels of the envelope signal. This implies in, errors in the measurement that is interpreted as $0^{\circ} \mathrm{C}$, this instant is indicated by a blue arrow in Fig. 36. Affer manually adjusting the threshold level the system operates without errors for approximately three hours and twenty minutes (black and red line coincide), without the need of any more none readjustments. The raw sensor data and the demodulated sensor data in this period measured the same values of the sensor. 
At the time marked by the green arrow, $3 \mathrm{~h}$ and $25 \mathrm{~m}$, in figure 4.36 , the demodulated sensor starts to provide wrong data. Since no further adjustments was made, the data still in $0^{\circ} \mathrm{C}$ during the rest of the test, orange rectangle in Fig. 4.36. However, the raw measurement still receiving data from the sensor, which means that the inside block is fully operating at this stage and has sufficient power to supply the electronics peripherals.

The main causes for the non-correct demodulation of the signal, both in the beginning of the experiment (blue arrow) as well as in its end (green arrow), can be associated with two main parameters observed in the experiment. The first is a slightly shiff on the frequency in which the highest V11 difference occurs. This was observed by monitoring the V11 difference along the time. One cause of this phenomenon is, probably caused by the variation of temperature, which affect solids[121] and liquids [122]. This causes a change on the levels of the envelope signal, which eventually drift it to out of the threshold level. The second is a phenomenon similar to cavitation [123] that generates bubbles in the water, with a mid-power $(10 \mathrm{~W})$ operation this phenomenon becomes present affer some time, and the waves behaves with high instability, impairing the behavior of the system. The Fig. 4.37 shows a picture with the bubbles inside the water, formed because of the cavitation-like phenomenon.

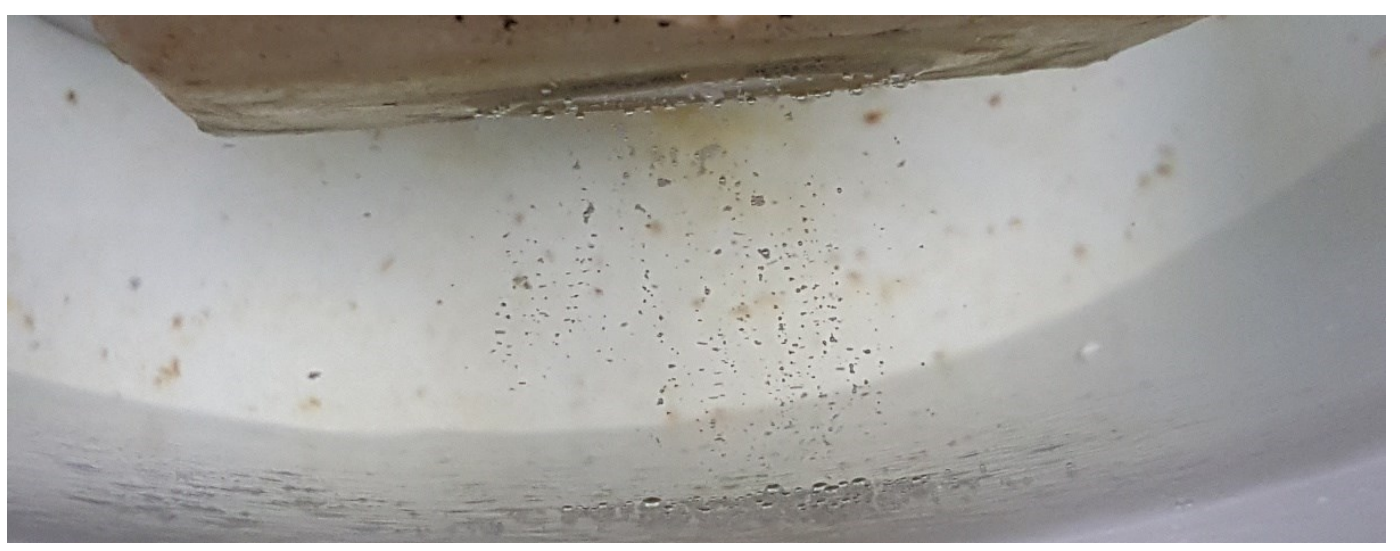

Figure 4.37 Bubblesgenerated inside the water due to cavitation-like phenomenon

As demonstrated by this experiment, using sufficient power on the outside block to supply energy for the inside block and by properly adjusting the threshold level on the voltage comparator, the system is capable to power all inside block and sensor and to demodulate the sensor information successfully. A critical point of this system is the proper adjustment of the comparator threshold level. In this experiment adjustment was done manually. A natural evolution of the present 
system is to count on an automatic selfadjusting logic unit to search for an optimum frequency.

\section{5 \\ Conclusion}

Analytical and numerical models were evaluated and extended. Simulation showed that the thickness of the adhesive and metal layer impacts on the power transfer efficiency. Increasing the thickness of the adhesive also increases the power loss. On the other hand, increasing the thickness of metal not necessarily increases the power loss, due to the resonances distribution in frequency. The search for the proper channel configuration is thus important and the models aid in this task.

Communication was assessed by simulating the input voltage (V11) of the system. The evaluation of data capacity was adopted in the frequency and time domains. The amplitude modulation of the input voltage, in transient response, shows a coherent behavior compared to the frequency response, and also shows the high selectivity of the channel when slightly varying the operating frequency.

When comparing the experiments with the two models used, the power transfer showed a coherent result with a great proximity in the peaks of resonance and anti-resonance. However this agreement was not clear in the communication analysis mainly in the curved wall-water configuration. Data communication analysis is affected by the complex impedance of the system. Pspice and analytical model have particular losses in their models, implying a different real and imaginary part of the equivalent impedance (Z11). A slightly shiff of the peaks in the V11 difference along the frequency spectrum between models is observed, see Fig. 4.29. The real case, on the other hand, may present some phenomena which are not included in neither of the models. For instance, the models do not consider any transverse wave or mode conversion. These may be present in the experiment, by means of non-perpendicular path, due to the transducer beam divergence, and the converted modes of indirect incidence. 
The system showed itself to be able to communicate with the sensor by amplitude modulating the carrier signal, generated in the "outside block", varying the electrical impedance load of the "inside" transducer. Specifically, concerning the curved wall-water configuration, in which the developed prototype were applied, the achieved results showed that the system can communicate with the sensor at a data rate of $9600 \mathrm{bps}$ when interrogated once per second. The power consumption of the "inside block" was calculated to be approximately $135 \mathrm{~mW}$, which is well below the capacity of the channel, measured at $1.4 \mathrm{~W}$ when setting the output power at $10 \mathrm{~W}$.

The last experiment compared the communication of a temperature sensor through the acoustic channel with raw sensor data, directed connected to a computer terminal. Communication was established successfully and the system kept running for approximately four hours. There were two events when it did not operated correctly and required manual intervention. It was caused mainly due to the high frequency selectivity of the acoustic channel.

Lastly, one can conclude that the experiments conducted in the laboratory, with a flat aluminum plate and curved carbon steel along with a water layer, has shown feasibility to communicate and power an isolated circuitry, in the "inside block", that comprises a low-power microcontroller and digital pressure and temperature sensor. Some identified that the system vulnerability when using wallwater configuration is possibily due to coaxial misalignment. between emitter and receiver transducers and acoustic velocities variation of the materials, which is caused by the dependence of temperature, inducing a dramatically attenuation of power and data communication capability.

Some points can be raised as future works. One can improve solutions for data demodulation by eliminating the analog voltage comparator and implementing the demodulation using digital signal processing techniques in microcontroller or a digital signal processor (DSP), which would render the system less sensitivity to noise. Additionally, dynamical tracking the optimum operating frequency avoids the systems increase of package loss. techniques like genetic algorithm or neural network may help predicting the best frequency to operate. Some other possible future work could be: 
- Studies regarding the cavitation-like phenomena to clarify the power transfer when the transducer is in contact with fluids [98], since it causes heat on the transducer and bubbles on the fluid with possibility of degradation.

- Extension and developing of numerical and analytical models focusing on the curved surface problem $[96,97]$ in order to understand power and data transfer behavior.

- Studies on the dependence of temperature and pressure on the system, mainly the adhesive material, since its acoustic properties starts degrading due to temperature variation [91].

- Studies on the impact of misalignment between transducers both circumferential as well as longitudinal.

- Studies on the acoustic reverberation in multiple layers and techniques in order to focus the acoustic energy between transmitter and receiver transducers [112]. 


\section{Bibliographic reference}

[1] Iman Khajenasiri, Abouzar Estebsari, Marian Verhelst, Georges Gielen, A Review on Internet of Things Solutions for Intelligent Energy Control in Buildings for Smart City Applications, In Energy Procedia, Volume 111, 2017, Pages 770779, ISSN 1876-6102

[2] J. D. Krauss, Electromagnetics, $4^{\text {th }}$ Edition, McGraw-Hill, 1992.

[3] Lawrence E. Kinsler, Fundamentals of Acoustics, $4^{\text {th }}$ Edition, 2009.

[4] T. Hosman, M. Yeary, J. K. Antonio and B. Hobbs, "Multi-tone FSK for ultrasonic communication," 2010 IEEE Instrumentation \& Measurement Technology Conference Proceedings, Austin, TX, 2010, pp. 1424-1429.

[5] J. D. Ashdown et al., "A full-duplex ultrasonic through-wall communication and power delivery system," in IEEE Transactions on Ultrasonics, Ferroelectrics, and Frequency Control, vol. 60, no. 3, pp. 587-595, March 2013. doi: 10.1109/TUFFC.2013.2600.

[6] Lawry, T.J.; Wilt, K.R.; Roa-Prada, S.; Ashdown, J.D.; Saulnier, G.J.; Scarton, H.A.; Das, P.K.; Gavens, A.J. A high-temperature acoustic-electric system for power delivery and data communication through thick metallic barriers. Proc. SPIE2011.

[7] S. Roa Prada, Modeling, Design and Temperature Characterization of an Ultrasonic through-Wall Communication System. Order No. 3504526, Rensselaer Polytechnic Institute, Ann Arbor, 2010.

[8] Primerano, R.; Wanuga, K.; Dorn, J.; Kam, M.; Dandekar, K. EchoCancellation for Ultrasonic Data Transmission through a Metal Channel. In Proceedings of the 41st Annual Conference on Information Sciences and Systems, Baltimore, MD, USA, 14-16 March 2007; pp. 841-845.

[9] K. Wanuga, M. Bielinski, R. Primerano, M. Kam and K. R. Dandekar, "Highdata-rate ultrasonic through-metal communication," in IEEE Transactions on Ultrasonics, Ferroelectrics, and Frequency Control, vol. 59, no. 9, pp. 20512053, September 2012.

[10] Graham, D.J.; Neasham, J.A.; Sharif B.S. High bit rate communication through metallic structures using electromagnetic acoustic transducers. In Proceedings of the OCEANS 2009-EUROPE, Bremen, Germany, 11-14 May 2009; pp. 1-6.

[11] Sherrit, S.; Doty, B.; Badescu, M.; Bao, X.; Bar-Cohen, Y.; Aldrich, J.; Chang, Z. Studies of acoustic-electric feed-throughs for power transmission through structures. Proc. SPIE 2006, 6171. 
[12] Moss, S.; McMahon, P.; Konak, C.; Phoumasavanh, C.; Rajic, N.; Galea, S.; Powlesland, I. Modelling and Experimental Validation of the Acoustic Electric Feedthrough Technique; Air Vehicles Division: Canberra, Australia, 2008.

[13] Moss, S.; Phoumsavanh, C.; Konak, M.; Tsoi, K.; Rajic, N.; Galea, S.; Powlesland, I.; McMahon, P. Design of the acoustic electric feedth rough demonstrator mk-II. Annu. Rev. J. Inst. Mater. Eng. Aust. LTD 2009, 33, 187-200.

[14] Chase, R. Microcontroller Based Handheld Acoustic Communication \& Power Delivery through Metallic Barriers; Rensselaer Polytechnic Institute: Niskayuna, NY, USA, 2013.

[15] D. J. Graham, J. A. Neasham and B. S. Sharif Investigation of Methods for Data Communication and Power Delivery Through Metals, in IEEE

Transactions on Industrial Electronics, vol. 58, no. 10, pp. 4972-4980, Oct. 2011.

[16] Lawry, T.J.; Saulnier, G.J.; Ashdown, J.D.; Wilt, K.R.; Scarton, H.A.; Pascarelle, S.; Pinezich, J.D. Penetration-free system for transmission of data and power through solid metal barriers. In Proceedings of the Military

Communications Conference, Baltimore, MD, USA, 7-10 November 2011; pp. 389-395.

[17] Chakraborty, S.; Wilt, K.R.; Saulnier, G.J.; Scarton, H.A.; Das, P.K. Estimating channel capacity and power transfer efficiency of a multi-layer acoustic-electric channel. Proc. SPIE 2013, 8753.

[18] M. A. Gutierrez-Estevez, U. Krueger, K. A. Krueger, K. Manolakis and V. Jungnickel, "Acoustic channel model for adaptive downhole communication over deep drill strings," 2013 IEEE International Conference on Acoustics, Speech and Signal Processing, Vancouver, BC, 2013, pp. 4883-4887.

[19] O. Imoru, A. Jassal, H. Polinder, E. Nieuwkoop, J. Tsado and A. A. Jimoh, "An Inductive Power Transfer through metal object," 2013 1st International

Future Energy Electronics Conference (IFEEC), Tainan, 2013, pp. 246-251.

[20] H. Zangl, A. Fuchs, T. Bretterklieber, M. Moser and G. Holler, "An Investigation on Wireless Communication and Power Supply Through Metal Tank Walls," 2008 IEEE Instrumentation and Measurement Technology Conference, Victoria, BC, 2008, pp. 1452-1457.

[21] H. Zangl, A. Fuchs, T. Bretterklieber, M. J. Moser and G. Holler, "Wireless Communication and Power Supply Strategy for Sensor Applications Within Closed Metal Walls," in IEEE Transactions on Instrumentation and Measurement, vol. 59, no. 6, pp. 1686-1692, June 2010.

[22] C. Liu, A. P. Hu and N. K. C. Nair, "Coupling study ofa rotary Capacitive Power Transfer system," 2009 IEEE International Conference on Industrial Technology, Gippsland, VIC, 2009, pp. 1-6. 
[23] Graham, D.J. Investigation of Methods for Data Communication and Power Delivery through Metals. Ph.D. Thesis, University ofNewcastle upon Tyne, Newcastle upon Tyne, UK, 2012.

[24] Balanis CA. Advanced Engineering Electromagnetics. 2nd ed. New York: John Wiley and Sons; 2012. p. 200.

[25] H. Quazi, Azizul \& L. Konrad, William. (1982). Underwater acoustic communication. Communications Magazine, IEEE. 20.24 - 30.

[26] Carovac, A., Smajlovic, F., \& Junuzovic, D. (2011). Application of Ultrasound in Medicine. Acta Informatica Medica, 19(3), 168-171.

[27] S. Arra, J. Leskinen, J. Heikkila and J. Vanhala, "Ultrasonic Power and Data Link for Wireless Implantable Applications," 2007 2nd International Symposium on Wireless Pervasive Computing, San Juan, 2007,

[28] Hu, Y.; Zhang, X.; Yang, J.; Jiang, Q. Transmitting electric energy through a metal wall by acoustic waves using piezoelectric transducers. IEEE Trans.

Ultrason. Ferroelectr. Freq. Control 2003, 50, 773-781.

[29] Yang, Z.T.; Guo, S.H. Energy trapping in power transmission through a circular cylindrical elastic shell by finite piezoelectric transducers. Ultrasonics 2008, 48, 716-723.

[30] Yang, Z.; Guo, S.; Yang, J. Transmitting electric energy through a closed elastic wall by acoustic waves and piezoelectric transducers. IEEE Trans.

Ultrason. Ferroelectr. Freq. Control 2008, 55, 1380-1386.

[31] Hu, H.; Hu, Y.; Chen, C. Wireless energy transmission through a thin metal wall by shear wave using two piezoelectric transducers. In Proceedings of the IEEE Ultrasonics Symp osium, B eijing, China, 2-5 November 2008; pp. 21652168 .

[32] Hu, H.; Hu, Y.; Chen, C.; Wang, J. A system of two piezoelectric transducers and a storage circuit for wirel ess energy transmission through a thin metal wall. IEEE Trans. Ultrason. Ferroel ectr. Freq. Control 2008, 55, 231 2-2319.

[33] Sherrit, S.; Badescu, M.; Bao, X.; Bar-Cohen, Y.; Chang, Z. Efficient electromechanical network model for wireless acoustic-electric feed-throughs. Proc. SPIE 2005, 5758, 362-372.

[34] Sherrit, S. ; Doty, B.; Badescu, M.; Bao, X.; Bar-Cohen, Y.; Aldrich, J.; Chang Z. Studies of acoustic-electric feed-throughs fr power transmission through structures. Proc. SPIE 2006, 6171.

[35] Bao, X.; Biederman, W.; Sherrit, S. ; Badescu, M.; Bar-Cohen, Y.; Jones, C.; Aldrich, J.; Chang, Z. High-power piezoelectric acoustic-electric power feedt hru for metal walls. Proc. SPIE 2008, 6930.

[36] Sherrit, S. ; Bao, X.; Badescu, M.; Aldrich, J.; Bar-Cohen, Y.; Biederman, W.; Chang, Z. 1 kW Power Transmission Using Wireless Acoustic-Electric Feedthrough 
(WAEF). In Earth \& Space 2008: Engineering, Science, Construction, and Operations in Challen ging Environments; American Soci ety of Civil Engi neers: Long Beach, CA, USA, 2008; pp. 1-10.

[37] Moss, S.; McMahon, P.; Konak, C.; Phoumasavanh, C.; Rajic, N.; Gal ea, S.; Powlesland, I. Modelling and Experimental Validation of the Acoustic Electric Feedt hrough Technique; Air Vehi cles Divisi on: Can berra,

Austral ia, 2008.

[38] Moss, S.; Konak, M.; Phoumsavanh, C.; Tsoi, K.; Powl esland, I. Acoustic Electric Feedthrough Demonstrator Mk-I; Air Vehicles Divisi on: Canberra, Australia, 2009; p. 24.

[39] Moss, S.; Phoumsavanh, C.; Konak, M.; Tsoi, K.; Rajic, N.; Galea, S.; Powlesland, I.; McMahon, P. Design of the acoustic el ectric feedthrough demonstrator mk-II. Annu. Rev. J. Inst. Mater. Eng. Aust. LTD 2009, 33, 187 200.

[40] Moss, S.; Skippen, J.; Konak, M.; Powlesland, I. Footprint Reduction for the Acoustic Electric Feedthrough Technique; Air Vehi cles Divisi on, Defence Science and Tech nol ogy Organi zation, Commonwealth of Australia: Canberra, Austral ia, 2010 .

[41] Hobart, E; All sup, G.; Hosom, D.; Baldasarre, T. Acoustic modem unit. In Proceedings of the OCEANS 2000 MTS/IEEE Conference and Exhibition, Provi dence, RI, USA, 11-14 Sept ember 2000; pp. 769-772.

[42] Payton, R.M. System for Acoustically Passing El ectrical Signals through a Hull. U. S. Patent 6, 625,084 B1, 23 Sept ember 2003.

[43] Murphy, T.L. Ultrasonic Digital Communication System for a Steel Wall Mul tipath Channel: Methods and Results; Rensselaer Polytechnic Institute: Niskayuna, NY, USA, 2006.

[44] Saulnier, G.J.; Scarton, H.A.; Gavens, A.J.; Shoudy, D.A.; Murphy, T.L; Wetzel, M.; Bard, S.; Roa-Prada, S. ; Das, P. Through-Wall C ommunicati on ofLowRate Digital Data Using Ultrasound. In Proceedings of the IEEE Ultrasonics Symp osium, Vancouver, BC, Canada, 2-6 October 2006; pp. 1385-1389.

[45] M.E. Ki ziroglou, D.E. Boyle, S.W. Wright, E.M. Yeatman, Acoustic power delivery to pipeli ne monit oring wireless sensors, Ultrasonics, Vol ume 77, 2017, Pages 54-60, ISSN 0041-624X

[46] Primerano, R.; Wanuga, K.; Dorn, J.; Kam, M.; Dandekar, K. EchoCancellation for Ultrasonic Data Transmission through a Metal Channel. In Proceedings of the 41st Annual Conference on Information Sciences and Systems, B altimore, MD, USA, 14-16 March 2007; pp. 841-845. 
[47] Primerano, R.; Kam, M.; Dandekar, K. High bit rate ultrasonic communicati on through metal channels. In Proceedings of the 43rd Annual Conference on Informati on Sciences and Systems, B altimore, MD, USA, 18-20 March 2009; pp. 902-906.

[48] Primerano, R.A. High Bit-Rate Digital Communication through Metal Channels. Ph.D. Thesis, Drexel University, Philadelphia, PA, USA, 2010.

[49] Bi elinski, M.; Wanuga, K.; Primerano, R.; Kam, M.; Dandekar, KR. Application of Adaptive OFDM Bit Loading for High Dat a Rate Through-Metal Communication. In Proceedings of the 2011 IEE E Global Telecommu nications Conference, Houston, T X, USA, 5-9 December 2011; pp. 1-5.

[50] Wanuga, K.; Bielinski, M.; Primerano, R.; Kam, M.; Dandekar, K.R. Highdata-rate ultrasonic through-metal communication. IEEE Trans. Ultrason. Ferroel ectr. Freq. Control 2012, 59, 2051-2053.

[51] Bi elinski, M.; Wanuga, K.; Sosa, G.; Primerano, R.; Kam, M.; Dandekar, K.R. Transceiver Design 6r High Data Rate Through-Metal Communication in Naval Applications. Nav. Eng. J. 2013, 125, 121-126.

[52] Hosman, T.; Yeary, M.; Antonio, J.K. Design and Characterization of an MFSK-B ased Transmitter/Receiver for Ultrasonic Communication Through Metalli c Structures. IEEE Trans. Instrum. Meas. 2011, 60, 3767-3774.

[53] Kl uge, M. ; Becker, T.; Schalk, J.; Otterpohl, T. Remote acousti c powering and data transmission for sensors insi de of conductive envel opes. In Proceedings of the 2008 IE EE Sensors, Lecce, Italy, 26-29 October 2008; pp. 41-44.

[54] Kl uge, M. ; Sabater, J.; Schalk, J.; Ngo, L.V.; Seidel, H. ; Schmi d, U. Wireless Sensing of Physical Parameters Inside Hermetically Enclosed Conductive Envelopes. In Proceedings of the ASME 2007 International Design E ngineering Technical Conferences and Computers and Information in E ngineering Conference, Las Vegas, NV, USA, 4-7 September 2007; pp. 353-359.

[55] Ngo, L.V.; Kluge, M.; Sabater, J.; Schalk, J.; Seidel, H.; Schmid, U. LongTerm Performance of Ultrasonic Transducers Used for Energy and Data Transmission. In Proceedings of the 2008 2nd E uropean Conference Exhibition on Integration Issues of Miniaturized Systems, Barcelona, Spain, 9-10 April 2008; pp. 1-6.

[56] Shoudy, D.A.; Saulnier, G.J.; Scart on, H.A.; Das, P.K.; Roa-Prada, S.; Ashdown, J.D. ; Gavens, A. J. An Ultrasonic Through-Wall Communi cation System with Power Harvesting. In Proceedings of the IEEE Ultrasoni cs Symposium, New York, NY, USA, 28-31 October 2007; pp. 1848-1853.

[57] Wilt, K.R.; Scarton, H.A.; Roa-Prada, S.; Saulnier, G.J.; Ashdown, J.D.; Lawry, T. J.; Das, P.K. Gavens, A.J. Finite Element Modeling And Simulation of A Two-T ransducer Through-Wall Ultrasonic Communication System. In Proceedings of the ASME 2009 International Mechanical Engineering Congress and Expositi on, Lake Buena Vista, FL, USA; 2009; pp. 579-589. 
[58] Wilt, K.R.; Lawry, T.J.; Scarton, H. A.; Roa-Prada, S.; Saulnier, G.J.; Ashdown, J.D. ; Das, P.K.; Pinezich, J.D. Mechani cal Design Impli cations on Power Transer through Thick Metallic Barriers Using Piezoelectric Transducers. In Proceedings of the ASME 2010 International Mechanical Engineering Congress \& Exposition, Vancouver, BC, Canada, 12-18 November 2010; pp. 173-182.

[59] Lawry, T.J.; Wilt, K.R.; S carton, H.A.; Saulnier, G.J. Anal yt ical modeling of a sandwiched plate piezoelectric transformer-based acoustic-electric transmission channel. IEEE Trans. Ultrason. Ferroel ectr. Freq. Cont rol 2012, 59, 2476-2486.

[60] Lawry, T.J.; Wilt, K. R.; Roa-Prada, S.; Ashdown, J.D.; Saulnier, G.J.; Scarton H.A.; Das, P. K.; Pinezich, J. D. Electrical optimization of power delivery through thick steel barriers using piezoelectric transducers. Proc. SPIE 2010, 7683.

[61] Lawry, T.J.; Wilt, K.R.; Roa-Prada, S.; Ashdown, J.D.; Saulnier, G.J.; Scarton, H.A.; Das, P.K.; Gavens, A.J. A high-temperature acoustic-electric system for power delivery and data communication through thick metall ic barriers. Proc. SPIE 2011, 8035 .

[62] Lawry, T.J.; Saulnier, G.J.; Ashdown, J.D.; Wilt, K.R.; Scarton, H.A; Pascarelle, S.; Pinezi ch, J.D. Penetration-free system fr transmission of dat a and power through solid metal barriers. In Proceedings of the Military Communicati ons Conference, Baltimore, MD, USA, 7-10 November 2011; pp. 389-395.

[63] Lawry, T. AHigh Performan ce System for Wireless Transmission of Power and Data Through Solid Metal Enclosures; Renssel aer Pol ytechnic Institute: Troy, NY, USA, 2011.

[64] Lawry, T.; Saulnier, G.; Wilt, K. Ashdown, J.; Scarton, H. Adapti ve System for Effici ent Transmissi on of Power and Data Through Acoustic Media. U.S. Patent 9,054, 826 B2, 28 March 2012.

[65] Lawry, T.J.; Wilt, K.R.; Ashdown, J.D.; Scarton, H. A.; Saulnier, G.J. A highperformance ul trasonic system for the simult aneous transmi ssion of data and power through sol id metal barriers. IEEE Trans. Ultrason. Ferroel ectr. Freq. Control 2013, 60, 194-203.

[66] Scarton, H. ; Saul ni er, G.; Wilt, K. Method and Apparatus for Acoustical Power Transfer and Communication. Patent WO 2,014,035, 785 A1, 6 March 2014.

[67] Chase, R. Microcontroll er Based Handheld Acoustic Communication \& Power Delivery through Metallic Barriers; Rensselaer Pol ytechnic Institute: Niskayuna, NY, USA, 2013.

[68] Chakraborty, S.; Wilt, K.R.; Saulnier, G.J.; Scart on, H.A.; Das, P.K. Est imating channel capacity and power transfer eff ciency of a multi-layer acoustic-electric channel. Proc. SPIE 2013, 8753. 
[69] Roa-Prada, S.; Scart on, H.A.; Saulnier, G.J.; Shoudy, D.A.; Ashdown, J.D.; Das, P.K.; Gavens, A.J. An Ultrasonic Through-Wall Communication (UT WC) Syst em Model. J. Vib. Acoust. 2013, 135.

[70] Graham, D.J.; Neasham, J.A.; Sharif, B.S. Investi gat ion of Methods br Data Communication and Power Delivery through Metals. IEEE Trans. Ind. Electron 2011, 58, 4972-4980.

[71] Graham, D.J.; Neasham, J.A.; Sharif, B.S. High bit rate communication through metallic structures using electromagnetic acoustic transducers. In Proceedings of the OCEANS 2009_EUR OPE, Bremen, Germany, 11-14 May 2009; pp. 1-6.

[72] W. M. Leach, Controlled-source analogous circuits and SPICE models for piezoelectric transducers, IEEE Trans. Ultrason. Ferroelectr. Freq. Control, vol. 41, no. 1, pp. 60-66, Jan. 1994.

[73] A. Püttmer, P. Hauptmann, R. Lucklum, O. Krause, and B. Henning, SPICE model for lossy piezoceramic transducers, IEEE Trans. Ultrason. Ferroelectr. Freq. Control, vol. 44, no. 1, pp. 60-66, Jan. 1997.

[74] J. van Deventer, T. Lofqvist, and J. Delsing, PSpice simulation of ultrasonic systems, IEEE Trans. Ultrason. Ferroelectr. Freq. Control, vol. 47, no. 4, pp. 1014-1024, Jul. 2000.

[75] K.R. Wilt, T.J. Lawry, H.A. Scarton, G.J. Saulnier, One-dimensional pressure transfer models for acoustic-electric transmission channels, In Journal of Sound and Vibration, Volume 352, 2015, Pages 158-173.

[76] Alan V. Oppenheim, Alan S. Willsky, and S. Hamid Nawab. 1996. Signals \&Amp; Systems (2nd Ed.). Prentice-Hall, Inc., Upper Saddle River, NJ, USA.

[77] Hayt, William. 1989. Engineering Electromagnetics (5th ed.), McGraw-Hill

[78] Dorf, R. C., \& Svoboda, J. A. (2004). Introduction to electric circuits (6th ed.). New York ; India: Wiley.

[79] H. Seki, A. Granato, and R. Truell, Diffaction Effects in the Ultrasonic Field of a Piston Source and Their Importance in the Accurate Measurement of Attenuation, J. Acoust. Soc. Am., vol. 28, no. 2, p. 230, 1956.

[80] R. Bass, Diffaction Effects in the Ultrasonic Field of a Piston Source, J. Acoust. Soc. Am., vol. 30, no. 7, p. 602, 1958.

[81] GmbH, P. (2017). Piezo Ceramic Technology, Piezo Actuators \& Piezo Components from PI Ceramic. [online] Piceramic.com. Available at: https://www.piceramic.com/en/ [Accessed 20 Sep. 2017].

[82] Huntsman.com. (2017). Huntsman Corporation. [online] Available at: http://www. huntsman.com/corporate/a/Home [Accessed 20 Sep. 2017]. 
[83] David R. Lide (ed), CRC Handbook of Chemistry and Physics, 84th Edition. CRC Press. Boca Raton, Florida, 2003; Section 14, Geophysics, Astronomy, and Acoustics; Speed of Sound in Various Media

[84] Sherrit, Stewart, Mukherjee, Binu K. Characterization of Piezoelectric Materials for Transducers, Dielectric and Ferroelectric Reviews, p. 175-244, 2012

[85] Pozar, D. M. (2005). Microwave engineering. Hoboken, NJ: J. Wiley.

[86] Anon, (2017). [online] Available at http://www. seas. upenn. edu/ jan/spice/PSpiceCaptureGuideOrCAD.pdf [Accessed 20 Sep. 2017].

[87] Leo, D. (2007). Engineering Analysis of Smart Material Systems. Hoboken: Wiley.

[88] Orcad.com. (2017). OrCAD PCB Solutions. [online] Available at: https://www.orcad.com/pt [Accessed 20 Sep. 2017].

[89] Sultan, N. W., Faget, J.-B. P., Fjeldheim, M., \& Sinet, J.-C. (2008, January 1). Real-Time Casing Annulus Pressure Monitoring in a Subsea HP/HT Exploration Well. Offshore Technology Conference. doi:10.4043/19286-MS

[90] Connor, D.J.; Cummings, G.F.; Star, M.J. Acoustic Transformer with NonPiezoelectric Core. U.S. Patent 5,594,705 A, 14 January 1997.

[91] T.P. Skourlis, R.L. McCullough, The effect of temperature on the behavior of the interphase in polymeric composites, In Composites Science and Technology, Volume 49, Issue 4, 1993, Pages 363-368, ISSN 0266-3538.

[92] D. Royer, Elastic waves in solids/ 2, Generation, acousto-optic interaction, applications. Springer, 2000.

[93] Mavko, G., Mukerji, T. and Dvorkin, J. (2009) The Rock Physics Handbook: Tools for Seismic Analysis of Porous Media

[94] K. Uchino and S. Hirose, Loss Mechanisms in Piezœel ectrics How to Measure Different Losses Separately, IEEE Trans. Ultrason., Ferroelectr., Freq. Con trol, vol. 48, pp. 307-321, Jan. 2001.

[95] D. A. Frickey, Conversions between S, Z, Y, H, ABCD, and T parameters which are valid for complex source and load impedances, in IEEE Transactions on Microwave Theory and Techniques, vol. 42, no. 2, pp. 205-211, Feb 1994.

[96] Yang, Z.; Guo, S.; Yang, J. Transmitting electric energy through a closed elastic wall by acoustic waves and piezoelectric transducers. IEEE Trans. Ultrason. Ferroelectr. Freq. Control 2008, 55, 1380-1386.

[97] Lü, C.F.; Yang, J.S.; Wang, J.; Chen, W.Q. Power transmission through a hollow cylinder by acoustic waves and piezoelectric transducers with radial polarization. J. Sound Vib. 2009, 325, 989-999. 
[98] Young, F.R. (1999). Cavitation, Imperial College Press, ISBN 1860941982, Singapore

[99] CADENCE, Appl. Note Obtain S-Parameter Data from the Probe Window, 2009.

[100] Nayfeh, T. H., Wheelis, W. B., \& Leslie, H. D. (1986, August 1). The FluidCompensated Cement Bond Log. Society of Petroleum Engineers. doi:10.2118/13044-PA

[101] Simon Haykin. 1989. An Introduction to Analog and Digital Communications. John Wiley \& Sons, Inc., New York, NY, USA.

[102] Bruce Carter, Thomas R. Brown. Handbook of Operational Amplifier Applications, Sep. 2016

[103] Razavi, B. (2008). Fundamentals of microelectronics. Hoboken, NJ: John Wiley \& Sons

[104] Linear.com. (2017). LT1117 - 800mA Low Dropout Positive Regulators Adjustable and Fixed 2.85V,3.3V, 5V - Linear Technology. [online] Available at: http://www.linear.com/product/LT 1117 [Accessed 26 Sep. 2017].

[105] GmbH, P. (2017). Piezo Ceramic Technology, Piezo Actuators \& Piezo Components from PI Ceramic. [online] Piceramic.com. Available at: https://www.piceramic. com/en/ [Accessed 26 Sep. 2017].

[106] Huntsman.com. (2017). Huntsman Corporation. [online] Available at: http://www.huntsman.com/corporate/a/Home [Accessed 26 Sep. 2017].

[107] Ounaies T.L. Jordan and Z.. 2001. Piezoelectric Ceramics Characterization. Technical Report. Institute for Computer Applications in Science and Engineering (ICASE).

[108] Tsai, Shu-Jen Steven, Power Transformer Partial Discharge (PD) Acoustic Signal Detection using Fiber Sensors and Wavelet Analysis, Modeling, and Simulation, Master thesis, 2006

[109] Comunicação, U. (2017). [online] Ultraceram.com.br. Available at: http://ultraceram.com.br/ [Accessed 26 Sep. 2017].

[110] Amry Amin Abas, Mohamad Pauzi Ismail, Suhairy Sani, \& Mohd Noorul Ikhsan Ahmed (2010). Effect of backing layer composition on ultrasonic probe bandwidth. RnD Seminar 2010: Research and Development Seminar 2010, Malaysia

[111] Dominique Paret and Carl Fenger. 1997. The I2c Bus: From Theory to Practice. John Wiley \& Sons, Inc., New York, NY, USA.

[112] M. Fink , D. Cassereau , A. Derode , C. Prada , P. Roux , M. Tanter, J. -L Thomas, and F. Wu , Time-reversed acoustics, Rep. Prog. Phys. 63(12), 19331995 (2000). 
[113] GmbH, P. (2017). Piezoceramic Materials. [online] Piceramic. com. Available at: https://www.piceramic.com/en/products/piezoceramic materials/\#c15162 [Accessed 26 Sep. 2017].

[114] Tek.com. (2017). TSG4100A RF Vector Signal Generator Datasheet | Tektronix. [online] Available at: http://www.tek.com/datasheet/tsg4100a-rfvectorsignal-generator-datasheet [Accessed 26 Sep. 2017].

[115] Rfamplifiers.eandiltd.com. (2017). 100W, $10 \mathrm{kHz}$ - $12 \mathrm{MHz}$ RF Amplifier. [online] Available at: http://rfamplifiers. eandiltd.com/item/rfamplifiers/rfamplifiers-1/21001/?\&cov=\&heading=\&tgid=\&tinid=\&appid=124 [Accessed 26 Sep. 2017].

[116] Tek.com. (2017). Keithley 2220/2230/2231 Multiple Output DC Power Supply | Tektronix. [online] Available at: http://www.tek.com/tektronix-andkeithley-dc-power-supplies/keithley-2220-2230-2231-series [Accessed 26 Sep. 2017].

[117] Openfield-technology.com. (2017). OpenField Technology | Openfield. [online] Available at: http://openfield-technology.com/ [Accessed 26 Sep. 2017].

[118] Ti.com. (2017). Analog, Embedded Processing, Semiconductor Company, Texas Instruments - TI.com. [online] Available at: http://www.ti.com [Accessed 26 Sep. 2017].

[119] Ftdichip.com. (2017). FT232R. [online] Available at: http://www.ffdichip.com/Products/ICs/FT232R.htm [Accessed 26 Sep. 2017].

[120] Ethik.com.br. (2017). ethik. [online] Available at: http://www. ethik.com.br/en/ [Accessed 26 Sep. 2017].

[121] Biagiotti, S. F. (1997, January 1). Effect Of Temperature On Ultrasonic Velocity In Steel. NACE International.

[122] K. Ikeda, "Ultrasonic measurement of sound velocity in liquids which automatically eliminates temperature dependence," 1998 IEEE Ultrasonics Symposium. Proceedings (Cat. No. 98CH36102), Sendai, 1998, pp. 1197-1202 vol.2.

[123] Herbert, Eric, Sébastien Balibar, and Frédéric Caupin. "Cavitation Pressure in Water." Physical Review E 74, no. 4 (October 16, 2006) : 041603. doi:10.1103/PhysRevE. 74.041603.

[124] Santos, R.; Orozco, J.; Micheletto, M.; Ochoa, S.F.; Meseguer, R.; Millan, P.; Molina, C. Real-Time Communication Support for Underwater Acoustic Sensor Networks. Sensors 2017, 17, 1629.

[125] Cañete, F. J., López-Fernández, J., García-Corrales, C., Sánchez, A., Robles, E., Rodrigo, F. J., \& Paris, J. F. (2016). Measurement and Modeling of Narrowband Channels for Ultrasonic Underwater Communications. Sensors (Basel, Switzerland), 16(2), 256. http://doi.org/10.3390/s16020256 
[126] B. Pranitha and L. Anjaneyulu, "Review of research trends in underwater communications - A technical survey," 2016 International Conference on Communication and Signal Processing (ICCSP), Melmaruvathur, 2016, pp. 1443-1447. doi: 10.1109/ICCSP.2016.7754395

[127] Booth RA, Goddard BA, Paton A. Measurement of fat thickness in man: a comparison of ultrasound, Harpenden calipers and electrical conductivity. British Journal of Nutrition. 1966;20(4):719-725.

[128] Bullen BA, Quaade F, Olessen E, Lund SA. Ultrasonic reflections used for measuring subcutaneous fat in humans. Human Biology. 1965;37(4):375-384. 